\title{
ASYMPTOTIC EXPANSIONS FOR INFINITE WEIGHTED CONVOLUTIONS OF HEAVY TAIL DISTRIBUTIONS AND APPLICATIONS
}

\author{
Ph. Barbe and W.P. McCormick \\ CNRS, France, and University of Georgia
}

\begin{abstract}
We establish some asymptotic expansions for infinite weighted convolution of distributions having regular varying tails. Various applications to statistics and probability are developed.
\end{abstract}

AMS 2000 Subject Classifications: Primary: 41A60, 60F99. Secondary: 41A80, 44A35, 60E07, 60G50, 60K05, 60K25, 62E17, 62G32.

Keywords: asymptotic expansion, regular variation, convolution, tail area approximation, ARMA models, tail estimation, randomly stopped sums, infinitely divisible distributions, renewal theory. 


\section{Contents}

1. Introduction

1.1. Prolegomenom

1.2. Mathematical overview and heuristics

1.3. Asymptotic scales

2. Main result

2.1. The Laplace characters

2.2. Smoothly varying functions of finite order

2.3. Asymptotic expansion for infinite weighted convolution

3. Implementing the expansion

3.1. How many terms are in the expansion?

3.2. Practical implementation: from Laplace's characters to linear algebra

3.3. Two terms expansion and second order regular variation

3.4. Some open questions

4. Applications

4.1. ARMA models

4.2. Tail index estimation

4.3. Randomly weighted sums

4.4. Randomly stopped sums

4.5. Queueing theory

4.6. Branching process

4.7. Infinitely divisible distributions

4.8. Implicit transient renewal equation and iterative systems

5. Proof in the positive case

5.1. Decomposition of the convolution into integral and multiplication operators

5.2. Organizing the proof

5.3. Regular variation and basic tail estimates

5.4. The fundamental estimate

5.5. Basic lemmas

5.6. Inductions

5.7. Conclusion

6. Removing the sign restriction on the random variables

6.1. Elementary properties of $U_{H}$

6.2. Basic expansion of $U_{H}$

6.3. A technical lemma

6.4. Conditional expansion and removing conditioning

7. Removing the sign restriction on the constants

7.1. Neglecting terms involving the multiplication operators

7.2. Substituting $\bar{H}^{(k)}$ and $\bar{G}^{(k)}$ by their expansions

8. Removing the smoothness restriction

Appendix. Maple code

References 
1. Introduction. The primary focus of this paper is to obtain precise understanding of the distribution tail of linear and related stochastic processes based on heavy tail innovations. In doing so, we will develop some new mathematical objects which are tailored to efficiently write and compute asymptotic expansions of these tails. Also, we will derive simple bounds of theoretical importance for the error between the tail and its asymptotic expansion.

These tails and their expansions are of interest in a variety of contexts. In the following subsection, we provide some typical examples to illustrate their use. The second subsection of this introduction overviews the new perspective and techniques developed in this paper; this will be done at a heuristic level, explaining the intuition and sketching the broad expanse wherein our methods lie. The last subsection contains basic facts on asymptotic scales.

1.1. Prolegomenom. The first basic problem we will deal with is related to the tail behavior of the marginal distribution of linear processes. To be specific, let $c=\left(c_{i}\right)_{i \in \mathbb{Z}}$ be a sequence of real constants, and let $X=\left(X_{i}\right)_{i \in \mathbb{Z}}$ be a sequence of independent and identically distributed random variables. Let $F$ be the common distribution function of these $X_{i}$ 's and write $\bar{F}=1-F$ the tail function. Assume that $F$ has a heavy tail, that is, for any positive $\lambda$,

$$
\lim _{t \rightarrow \infty} \bar{F}(t \lambda) / \bar{F}(t)=\lambda^{-\alpha}
$$

for some positive and finite $\alpha$. Let $G_{c}$ be the distribution function of the series $\sum_{i \in \mathbb{Z}} c_{i} X_{i}$. Set $\bar{G}_{c}=1-G_{c}$. For simplicity, assume in this introduction that the sequence $c$ is nonnegative. Define $C_{\alpha}=\sum_{i \in \mathbb{Z}} c_{i}^{\alpha}$. It is well known that under a mild additional condition,

$$
\bar{G}_{c} \sim C_{\alpha} \bar{F}
$$

at infinity, that is $\lim _{t \rightarrow \infty} \bar{G}_{c}(t) / \bar{F}(t)=C_{\alpha}$.

It is then a natural question to investigate higher order expansions. Under suitable conditions, we will obtain higher order asymptotic expansions both for $G_{c}$ and its derivatives. In particular all ARMA processes fall within the scope of our result, provided the innovation distribution satisfies certain mild conditions beyond that of heavy tail. 
This question of obtaining higher order terms has several motivations. One is that very little is known on the marginal distributions of linear processes, and since those are ubiquitous in statistics, better knowledge and understanding of their properties is desirable. There are also specific instances where higher order asymptotics are needed, such as in the tail index estimation problem which we will study in section 4.2. To achieve such refined distributional results in this setting, we must first build the mathematical language and theory needed for higher order expansions.

A third motivation comes from a large number of applications to related processes obtained by allowing the weights to be random. Whereas many first order results are known, few second order results exist, and no higher order results are available that we are aware of. We will obtain asymptotic expansions when the weights are random, not necessarily independent or identically distributed, but still assuming that the sequence $c$, now random, is independent of $X$.

This will allow us to derive tail expansions for compound sums, that is a sum with a random number of summands. As a consequence, we will derive a tail expansion for some infinitely divisible distributions. In the same vein, having a result with random weights yields expansions for tails arising in transient renewal theory, and even in implicit transient renewal theory. By the latter, we mean, for example, distributions defined implicitly as follows. Let $M, Q$ and $R$ be independent nonnegative random variables and suppose that $R$ has the same distribution as $Q+M R$. Given the distributions of $M$ and $Q$, this defines that of $R$ implicitly. We will obtain an asymptotic expansion for the distribution of $R$, when $Q$ has heavy tail and $M$ has enough moments.

Furthermore, it will be clear that by the algebraic paradigm of this paper, it is quite a simple matter to write out what the expansions should be, and often not so difficult to prove that those expansions are valid under some reasonable conditions.

In all the contexts mentioned, first order results exist under heavy tail assumptions, or more generally in the subexponential framework. By now, these results are well understood. We refer to the book by Bingham, Goldie and Teugels (1989) for a comprehensive study of these topics and Broniatowski and Fuchs (1995) for a different perspective on first order results for sums of independent and identically distributed random variables. Resnick (1986) is 
a good reference for many first order results obtained through a point process argument. In comparison, second order results are few. In the heavy-tail case papers which provide second order results for the sum of two random variables include Omey (1988), Geluk (1994), Geluk et al. (1997) and Geluk et al. (2000). With regard to subexponential distributions, second order work for a convolution of two such distributions may be found in Cline (1986), Cline (1987) and Geluk and Pakes (1991). We also mention that Geluk et al. (2000) shows a second order result for a sum of a finite number of independent and identically distributed random variables in the special case of the underlying distribution being a member of the Hall-Weissman class. Barbe and McCormick (2005) obtained second order result for the sum of a finite number of heavy tailed random variables when the distributions also possess a mild smoothness property. Using a different set of assumptions and for a different purpose, Borovkov and Borovkov (2003) obtained higher order expansions also for a finite number of summands.

In renewal theory, a little more is known. For instance, in recurrent renewal theory, higher order results are already in Feller (1971). But our technique is useful only in the transient case, for which far less is known. Chover et al. (1973) provides a first order result while Grübel (1987) gives a second order formula. In the implicit case, Goldie (1991) obtained second order formulas. However, our work complements nicely with Goldie's, in the sense that our results typically apply when his fail. The techniques used by Goldie (1991) build on those devised by Kesten (1973) and Grincevičius (1975), and make use of delicate Tauberian theory, while that of Grübel (1987) is the so-called Banach algebra technique. Our technique is radically different from these.

Grübel's (1987) result on renewal measures allows him to obtain a second order formula for the tail of some infinitely divisible distributions, using a decomposition which we could trace back at least to Feller (1971). We will use the same decomposition to obtain higher order results.

Since our approach to deriving these asymptotic expansions may appear very algebraic to analytically oriented minds, we feel compelled at this point to quote a result which can be proved in less than 5 minutes after understanding the statement of our main result (Theorem 2.3.1) and being familiar with our algebraic formalism. So assume that $F$ is the distribution function defined by 
$\bar{F}(t)=(a+b)^{-1}\left(a t^{-\alpha}+b t^{-\beta}\right)$ for $t$ more than 1 , and with $1<\alpha<\beta$. Assume further that the $c_{i}$ 's are nonnegative. Write $C_{r}=\sum_{i \in \mathbb{Z}} c_{i}^{r}$, and

$$
\mu_{F, 1}=\frac{1}{a+b}\left(\frac{a}{\alpha-1}+\frac{b}{\beta-1}\right)
$$

for the first moment of $F$. Then we have the two terms expansion

$$
\begin{aligned}
& \bar{G}_{c}(t)=\frac{a}{a+b} \frac{C_{\alpha}}{t^{\alpha}} \\
& +\left\{\begin{array}{ll}
\frac{b C_{\beta}}{a+b} \frac{1}{t^{\beta}} & \text { if } \beta<\alpha+1, \\
\frac{1}{a+b}\left(b C_{\beta}+a \mu_{F, 1} \alpha\left(C_{1} C_{\alpha}-C_{\alpha+1}\right)\right) \frac{1}{t^{\alpha+1}} & \text { if } \beta=\alpha+1, \\
\frac{a \alpha}{a+b} \mu_{F, 1}\left(C_{1} C_{\alpha}-C_{\alpha+1}\right) \frac{1}{t^{\alpha+1}} & \text { if } \alpha+1<\beta .
\end{array}\right\} \\
& +o\left(\frac{1}{t^{\alpha+1}}\right)
\end{aligned}
$$

With our formalism and notations to be explained later, this complicated formula has the far more manageable form, where no cases need to be distinguished,

$$
\bar{G}_{c} \sim \sum_{i \in \mathbb{Z}} L_{G_{c} \sharp M_{c_{i}} F, 1} \overline{M_{c_{i}} F} .
$$

Note that if $\beta$ is $\alpha+1$, the $c_{i}$ 's and $b$ can be chosen so that the above second order expansion yields only

$$
\bar{G}_{c}=\frac{a}{a+b} C_{\alpha} t^{-\alpha}+o\left(t^{-\alpha-1}\right) .
$$

It is then desirable to get an extra term. In section 3.1 , we will obtain this term, generically, when $\alpha$ is more than 2 . We will also explain why in some very exceptional cases, obtaining a second term is rather hopeless (last example of section 3.1), while in other cases, obtaining as many terms as one desires can be trivial (penultimate example in section 3.2).

To conclude this preliminary discussion, and perhaps to motivate further our investigation, we mention that there are many unsolved very basic problems related to linear processes. For instance, for discrete innovation, Davis and Rosenblatt (1991) show that all but trivial infinite order linear processes have a continuous marginal distribution; but there is still no good criterion to know if the 
marginal distribution of the process is absolutely continuous. Even in the most basic cases, the behavior of the marginal distribution is amazingly difficult to analyze. Solomyak's (1995) breakthrough a generic result on absolute continuity of the marginal distribution of first order autoregressive models with Bernoulli innovations gives a chilling reminder on how little is known in general. So this paper can be taken as a contribution to the understanding of those distributions, in the continuous and heavy tail situation.

1.2. Mathematical overview and heuristics. We now outline the mathematical content of the key parts of this paper and provide some intuition behind the main result.

In our opinion, the main reason why so few higher order results are available in the problems we are interested in, is that too much emphasis has been given to an analytical perspective. Our first basic remark is that the convolution operation $(F, G) \mapsto F \star G$ is bilinear and defines a semi-group. Bilinearity is a notion in linear algebra; while semi-group is related to the group structure, which in turn suggests representation theory. So our view is that the asymptotic behavior of the convolution semi-group should be analyzed by a linear representation which captures only the tail behavior of the semi-group. In fact we will obtain two linear representations, one which is suitable for writing the expansions, the other one, derived from the first one, and requiring more assumptions, suitable for practical computations.

To explain further our view on the subject, let us give a heuristic argument on how to derive higher order expansions and build the mathematical language to handle these expansions.

Heuristic. Let $F$ and $G$ be two distribution functions. Their convolution is

$$
\overline{F \star G}(t)=\int \bar{F}(x-y) \mathrm{d} G(y) .
$$

The left hand side in this formula is symmetric, while the right hand side, as far as the notation goes, is not. To symmetrize it, we split the integral and integrate by parts to obtain

$\overline{F \star G}(t)=\int_{-\infty}^{t / 2} \bar{F}(t-x) \mathrm{d} G(x)+\int_{-\infty}^{t / 2} \bar{G}(t-x) \mathrm{d} F(x)+\bar{F}(t / 2) \bar{G}(t / 2)$.

This formula involves the translation $\tau_{x}$ acting on functions and defined by $\tau_{x} h(t)=h(t+x)$. The translations form a semigroup 
since $\tau_{x} \tau_{y}=\tau_{x+y}$. The corresponding infinitesimal generator is the derivative $\mathrm{D}$, because for smooth functions

$$
\lim _{\epsilon \rightarrow 0} \frac{\tau_{\epsilon}-\tau_{0}}{\epsilon} h(t)=\lim _{\epsilon \rightarrow 0} \frac{h(t+\epsilon)-h(t)}{\epsilon}=\mathrm{D} h(t) .
$$

So, one expects to be able to write $\tau_{x}=e^{x \mathrm{D}}$ — this is nothing but a neat way to write a Taylor expansion, which we could trace back to Delsarte (1938). Indeed, applying both sides to a smooth function $h$ yields

$$
h(t+x)=\tau_{x} h(t)=e^{x \mathrm{D}} h(t)=\sum_{i \geqslant 0} \frac{x^{i} \mathrm{D}^{i}}{i !} h(t)=\sum_{i \geqslant 0} \frac{x^{i}}{i !} h^{(i)}(t) .
$$

So, formally,

$$
\int_{-\infty}^{t / 2} \bar{F}(t-x) \mathrm{d} G(x)=\int_{-\infty}^{t / 2} \tau_{-x} \mathrm{~d} G(x) \bar{F}(t)=\int_{-\infty}^{t / 2} e^{-x \mathrm{D}} \mathrm{d} G(x) \bar{F}(t) .
$$

As $t$ tends to infinity, the linear operator $\int_{-\infty}^{t / 2} e^{-x \mathrm{D}} \mathrm{d} G(x)$ should tend to the Laplace transform of $G$ at D, say $L_{G}(\mathrm{D})$. So, ideally, we have

$$
\overline{F \star G}=L_{G}(\mathrm{D}) \bar{F}+L_{F}(\mathrm{D}) \bar{G}+O(\bar{F} \bar{G}) .
$$

Of course, for heavy tail distributions, many things are not well defined. Certainly, the Laplace transform does not exist, the distribution lacking sufficient integrability.

So, consider the Taylor polynomial of the Laplace transform. Write $\mu_{G, k}$ for the $k$-th moment of $G$. Assume that these moments are finite for $k$ at most equal to some $m$. The Taylor polynomial of the Laplace transform gives rise to the differential operator

$$
L_{G, m}=\sum_{0 \leqslant j \leqslant m} \frac{(-1)^{j}}{j !} \mu_{G, j} \mathrm{D}^{j} .
$$

Assume that $\bar{F}$ and $\bar{G}$ are regularly varying of index $-\alpha$, that is obey (1.1.1). Take $m$ be an integer less than $\alpha$. Throughout this paper, Id denotes the identity function of whatever space is under consideration. When using the identity on the real line, we write $\mathrm{Id}^{k}$ for the function $t \mapsto t^{k}$. So, $\mathrm{Id}^{-m} \bar{F}$ is the function whose value at $t$ is $t^{-m} \bar{F}(t)$. We certainly would like to have the $m$-th 
derivative $\bar{F}^{(m)}$ regularly varying of index $-\alpha-m$. In that case $\left(L_{G}-L_{G, m}\right) \bar{F}$ could be of order smaller than the last term, that is $\bar{F}^{(m)}$, or equivalently, $\mathrm{Id}^{-m} \bar{F}$, and therefore dominates $\bar{F} \bar{G}$. So replacing $L_{G}(\mathrm{D})$ and $L_{F}(\mathrm{D})$ in (1.2.1) by $L_{G, m}$ and $L_{F, m}$ and taking $m$ less than $\alpha$ suggests

$$
\overline{F \star G}=L_{G, m} \bar{F}+L_{F, m} \bar{G}+o\left(\operatorname{Id}^{-m} \bar{F}\right) .
$$

The advantage of this substitution is that now everything is well defined, in particular it does not assume that $F$ and $G$ have moments of arbitrary large order.

By induction, if we have $n$ distribution functions $F_{i}, 1 \leqslant i \leqslant n$, we obtain

$$
\overline{\star_{1 \leqslant i \leqslant n} F_{i}}=\sum_{1 \leqslant i \leqslant n} L_{\star_{\substack{1 \leqslant j \leqslant n \\ j \neq i}} F_{j}, m} \bar{F}_{i}+\text { remainder. }
$$

Taking limit at infinity yields a formula, which, restricted to the context of linear processes, is in fact the main result of this paper.

The substantial task of transforming this heuristic argument into a rigorous proof will be carried out in sections 5, 6, 7 and 8 .

Going back to our heuristic, where is the representation which we announced? We will show that in the proper ring, that of polynomials in $\mathrm{D}$ modulo the ideal generated by $\mathrm{D}^{m+1}$, the composition of $L_{F, m}$ with $L_{G, m}$ gives $L_{F \star G, m}$. So the map $F \mapsto L_{F, m}$ turns out to be a linear representation of the convolution algebra in a ring of differential operators. In this ring, the operators $L_{F, m}$ will be invertible.

This gives us a very powerful formalism to state expansions. But, arguably, it is not quite explicit. To obtain a powerful computational machinery, we need to go from a ring of differential operators to a vector space where we can manipulate finite dimensional matrices. This is not always possible though, and it is related to a rather intricate matter concerning asymptotic scales. Those will be introduced in the next subsection and further examined in our context in subsections 3.1 and 3.2.

Nevertheless, the basic idea is quite intuitive. For any positive real number $c$, write $M_{c} F$ to denote the distribution function of $c X_{i}$. More generally, when $c$ is positive and $h$ is a function, we write $M_{c} h$ for the function whose value at $t$ is $h(t / c)$. Since $\mathrm{D} \overline{M_{c} F}=c^{-1} M_{c} \mathrm{D} \bar{F}$, 
the expansion (1.2.2) with $m=1$ yields

$$
\begin{aligned}
\overline{M_{c_{1}} F \star M_{c_{2}} F}=M_{c_{1}} \bar{F}+ & M_{c_{2}} \bar{F}-c_{1} \mu_{F, 1} c_{2}^{-1} M_{c_{2}} \mathrm{D} \bar{F} \\
& -c_{2} \mu_{F, 1} c_{1}^{-1} M_{c_{1}} \mathrm{D} \bar{F}+\text { remainder. }
\end{aligned}
$$

This suggests that we could think of such expansion as a decomposition in a basis comprised of $\bar{F}$ and $\mathrm{D} \bar{F}$, that is as a sort of projection onto the 2-dimensional vector space spanned by these functions. But there is a problem to overcome, namely that the sum $M_{c_{1}} \bar{F}+M_{c_{2}} \bar{F}$ is not quite the usual first order result $\left(c_{1}^{\alpha}+c_{2}^{\alpha}\right) \bar{F}$. In doing such a replacement, we make an error, which may be larger than the second order term. So, even in an asymptotic sense, $M_{c} \bar{F}$ may not be in the space spanned by $\bar{F}$ and $\mathrm{D} \bar{F}$. We will give examples in sections 3.1 and 3.2 showing incidentally that this problem is not a failure of the expansion, but that of the basis $\bar{F}, \mathrm{D} \bar{F}$. This suggests that some bases are better than others, and of course raises the question of what is a good basis for our purpose.

Since we will now be dealing with issues related to asymptotic analysis, we will switch to a terminology used in that field. Specifically, we will refer to an asymptotic scale for that which we called a basis in the previous paragraph. Asymptotic scales will be introduced formally in the next subsection.

In studying linear processes, two operations are involved. One is the convolution obtained from summing independent random variables, the other is the scaling $M_{c}$ obtained by rescaling the variables. We have seen that as far as the convolution goes, we have a representation using the differential operators $L_{F, m}$, which themselves involve D. This suggests that the good asymptotic scales are in some sense stable by differentiation and rescaling. In which sense? Since we are interested in asymptotic expansions, and we want to preserve the linear aspect of the convolution, they should be stable in the sense that derivative and scaling of functions in this scale should have an asymptotic expansion in the same scale. The simplest example of such functions are the powers $t^{-\alpha-n}, n \in \mathbb{N}$. We will see many others. We call such stable scale a $\star$-asymptotic scale, and that will be defined formally in subsection 3.2 .

So, assume that $e=\left(e_{i}\right)_{i \in I}$ is a set of functions defining our $\star$ asymptotic scale. Then there exists a matrix $\mathcal{D}=\left(\mathcal{D}_{i, j}\right)_{i, j \in I}$, such that for any $i$ in $I$, the derivative $\mathrm{D} e_{i}$ has asymptotic expansion $\sum_{j \in I} \mathcal{D}_{i, j} e_{j}$; and for any positive $c$, there exists a matrix $\mathcal{M}_{c}$, such 
that $M_{c} e_{i}$ has asymptotic expansion $\sum_{j \in I}\left(\mathcal{M}_{c}\right)_{i, j} e_{j}$. In particular, defining the matrix

$$
\mathcal{L}_{G}=\sum_{0 \leqslant j \leqslant m} \frac{(-1)^{j}}{j !} \mu_{G, j} \mathcal{D}^{j}
$$

we see that $L_{G, m} e_{i}$ has asymptotic expansion $\sum_{j \in I}\left(\mathcal{L}_{G}\right)_{j, i} e_{j}$. Since the differential operators $L_{G, m}$ are representations of the convolution semi-group, so are the matrices $\mathcal{L}_{G}$. This is our second representation of the convolution semigroup, which is now made up of finite dimensional matrices. Note that compared to the first representation using differential operators, we assume the existence of a $\star$-asymptotic scale in which functions can be expanded. We will see that for usual distributions this is not a restriction at all, and it is even very natural and desirable. Also, the representation $L_{F, m}$ has dimension $m+1$, while that given by $\mathcal{L}_{F}$ will often be of higher dimension.

Now, if $\bar{F}$ has an asymptotic expansion $\sum_{i \in I} p_{\bar{F}, i} e_{i}$, this expansion is encoded in the vector $p_{\bar{F}}=\left(p_{\bar{F}, i}\right)_{i \in I}$. Then, the asymptotic expansion of $\overline{M_{c} F}$ is encoded in the vector $\mathcal{M}_{c} p_{\bar{F}}$, which is simply a product of a matrix by a vector. Thus one can hope that because $L_{G, m}$ is a linear operator, $L_{G, m} \bar{F}$ has an asymptotic expansion encoded by the vector is $\mathcal{L}_{G} p_{\bar{F}}$. In particular, formula (1.2.3) can be written as

$$
p_{\overline{M_{c_{1}} F \star M_{c_{2}} F}}=\left(\mathcal{L}_{M_{c_{1}} F} \mathcal{M}_{c_{2}}+\mathcal{L}_{M_{c_{2}} F} \mathcal{M}_{c_{1}}\right) p_{\bar{F}} .
$$

This means that we can calculate the asymptotic expansion of $M_{c_{1}} F \star M_{c_{2}} F$ in a $\star$-asymptotic scale using multiplication and addition of matrices and vectors. The advantage of using this matrix representation is that we are now in the realm of linear algebra, where computers can be used both to do numerical and formal work. This is the key to efficient derivations of expansions in practical cases. One last point worth mentioning with regard to this algebraic approach is that the complexity of argument to obtain a higher order expansion is essentially the same as that to obtain a second order expansion. The effect of higher order is to raise the dimension of the space one works with; but, for second order and beyond, the spaces used will all have dimension at least 2. Only a first order result for which a 1-dimensional space suffices can attain a significant reduction in 
effort. In the same vein, we note that expansions on densities and their derivatives are obtained with little extra effort. Furthermore, these results are useful, for example, in discussing the Von Mises condition for infinite order weighted averages - see subsection 4.2.

Of course, all this heuristic elaboration is rather formal at this point, and clearly the realization of this program requires us to define the proper analytical setup - this will be done in subsection 2.1 as well as the algebraic machinery - to be done in part in subsection 2.2 - simply to be able to state the proper theorem in subsection 2.3 .

Up to now we have mainly presented the algebraic formalism. It is clear that in order to justify rigorously these heuristic arguments, one has to connect this formalism to analysis. As mentioned earlier, this is mostly done in sections 5,6 and 7. At this point, it is not useful to outline the proof; such outline is given in section 5.2. But we mention that the proof is by induction on the number of summands, and then a limiting argument as the number of summands goes to infinity. Of course, any limiting argument in this type of asymptotics requires a good error bound between the original functions and their approximations, while induction somewhat requires tractable bounds. Those two requirements, sharpness and simplicity, tend to be in opposition. The key to obtain both features will be a functional analytic approach. We will write the convolution in terms of some operators, and the study of those operators will give us good bounds.

The many steps alluded to in our heuristic arguments may suggest that particularly severe restrictions will be needed on the distribution function of the $X_{i}$ 's and the constants $c$. However, this is not the case and the main result is rather sharp. Before going any further, we mention that our asymptotic expansions involve derivatives and moments. It will be clear from the proof that some regularity of the underlying distribution must be assumed in order to obtain even a two terms expansion. The regularity assumption has a great impact on the form of the expansion. The assumption chosen hereafter, namely differentiability up to a certain order, seems the most natural one for applications to concrete distributions. In the same vein, Barbe and McCormick (2005) show that when $\alpha$ is 1 and the first moment is infinite, the truncated first moment is involved in a two terms expansion for finite convolution; moreover, the expansion takes a very different form if $\alpha$ is less than 1 . It is quite clear from Barbe 
and McCormick (2005) how some results of the present paper can be formally modified when one does not have enough moments. The overall philosophy of this work is to prove that in a natural setting, limit as the number of summands tends to infinity and differentiation can be permuted with asymptotic expansions. It is however a daunting task - if not hopeless - to prove a general theorem which could cover all possible cases. In particular, it is useful to remember that even for the convolution of two heavy tail distributions, there is no known second order formula that covers every possible case; however, we will provide some formulas or techniques which cover most distributions of interest in applications, if not all.

1.3. Asymptotic scales. To conclude this introductory section, we present a few facts on terminology and notations related to asymptotic expansions. We refer to Olver (1974) for a complete account.

Let $N$ be either a positive integer or $+\infty$. A family of functions $e_{i}$, $0 \leqslant i<N$, is called an asymptotic scale if whenever $i+1$ is less than $N$, the asymptotic relation $e_{i+1}=o\left(e_{i}\right)$ - i.e. $e_{i+1}(t)=o\left(e_{i}(t)\right)$ as $t$ tends to infinity - holds. We say that a function $f$ has an $(n+1)$-terms asymptotic expansion in the scale $\left(e_{i}\right)_{0 \leqslant i<N}$ if there exist real numbers $f_{i}, 0 \leqslant i \leqslant n$ such that

$$
f(t)=\sum_{0 \leqslant i \leqslant n} f_{i} e_{i}(t)+o\left(e_{n}(t)\right)
$$

as $t$ tends to infinity. We then write

$$
f \sim \sum_{0 \leqslant i \leqslant n} f_{i} e_{i}
$$

When $n$ vanishes, this notation agrees with the more usual one when one writes $f \sim g$ to mean that $f / g$ tends to 1 at infinity. Sometimes, it will be more convenient to index asymptotic scale by an ordered set, and an obvious variation of the definition will be understood.

However, this definition is insufficient for our purpose. The next step, following Olver (1974), is to introduce the notion of generalized asymptotic expansion with respect to an asymptotic scale $e_{i}, 0 \leqslant i<N$. If there are functions $\phi_{s}, 0 \leqslant s \leqslant n$ such that for any nonnegative integer $k$ at most $n$,

$$
f(t)=\sum_{0 \leqslant i \leqslant k} \phi_{i}(t)+o\left(e_{k}(t)\right)
$$


we say that $\sum_{0 \leqslant i \leqslant n} \phi_{i}(t)$ is a $(n+1$-terms $)$ generalized expansion with respect to the scale $e_{i}, 0 \leqslant i<N$, and the developing functions $\phi_{i}$. We write

$$
f \sim \sum_{0 \leqslant i \leqslant n} \phi_{i} \quad\left(e_{n}\right)
$$

or simply

$$
f \sim \sum_{0 \leqslant i \leqslant n} \phi_{i}
$$

if the scale is understood. Note that there is no a priori understanding that $e_{k}=o\left(\phi_{i}\right)$ for any $i$ between 0 and $n$; in theory, it could be that the only information conveyed in the expansion is that $f=o\left(e_{k}\right)$.

It is important to note that once the asymptotic scale is chosen, the asymptotic expansion of a given function in that scale, if it exists, is unique. However, a generalized expansion may not be unique, even if one fixes the functions $\phi_{i}$.

One should also be aware that the dependence of an asymptotic expansion on the asymptotic scale may be an important matter. For instance, write $\bar{\Phi}$ for the tail of the standard normal distribution and consider the asymptotic scale $e_{i}=\bar{\Phi}^{i}, i \in \mathbb{N}^{*}$. Define the function $f(t)=\bar{\Phi}(t)+\bar{\Phi}^{2}(t)$. One has the asymptotic expansion

$$
f \sim e_{1}+e_{2} .
$$

Clearly, this approximation cannot be improved. On the other hand, if one decides to use the asymptotic scale $\tilde{e}_{i}(t)=\bar{\Phi}(t) / t^{i}$, $i=0,1,2, \ldots$, one has for any nonnegative $n$

$$
f \sim \tilde{e}_{0} \quad\left(\tilde{e}_{n}\right)
$$

In this asymptotic expansion the part of $f$ coming from $e_{2}$ is not taken into consideration, despite the fact that in the chosen asymptotic scale, the expansion has as many terms as one wishes. Finally, if one decides to use the asymptotic scale $\bar{e}_{i}(t)=e^{-t^{2} / 2} / t^{i} \sqrt{2 \pi}, i \geqslant 1$, then

$$
f \sim \bar{e}_{1}+\sum_{i \geqslant 1}(-1)^{i} \frac{(2 i-1) !}{2^{i-1}(i-1) !} \bar{e}_{2 i+1} .
$$

Again, in the asymptotic scale $\bar{e}_{i}, i \geqslant 1$, this asymptotic expansion has as many terms as one wishes though the term in $e_{2}$ in $f$ is not 
taken into consideration. The term $e_{2}$ is effectively 0 at every level of scale with respect to the asymptotic scale $\left(\tilde{e}_{i}\right)_{i \geqslant 0}$ or the asymptotic scale $\left(\bar{e}_{i}\right)_{i \geqslant 1}$. Moreover, this last asymptotic expansion is a divergent series, which gives a useless approximation if one does not truncate it.

We highly recommend the short first chapter of Olver's (1974) book, where many simple examples are discussed; very useful, not so useful and totally useless expansions are shown; and the pros and cons of asymptotic expansions are well presented.

\section{Main result.}

To state our main result, we need to introduce two objects: the class of distribution functions we are dealing with and some differential operators which play a role in writing the asymptotic expansions. This is the purpose of the next two subsections. The main result of this paper is given in the third subsection.

Let us now introduce some notation and conventions.

We write $\mathbb{I}\{\cdot\}$ to denote the indicator function of a set $\{\cdot\}$.

Throughout this paper we let $M_{c}$ denote the multiplication operator on distribution functions corresponding to the multiplication of random variables by $c$. Hence, if $F$ is a distribution function and $X$ is distributed according to $F$,

$$
M_{c} F(t)=P\{c X \leqslant t\}= \begin{cases}F(t / c) & \text { if } c>0 \\ \bar{F}(t / c-) & \text { if } c<0 \\ \mathbb{I}\{t \geqslant 0\} & \text { if } c=0\end{cases}
$$

In the sequel, all distribution functions will be assumed continuous, thereby obviating the need for left limit notation. Note that for $c$ positive, $\overline{M_{c} F}=M_{c} \bar{F}$, while for $c$ negative, $\overline{M_{c} F}=M_{-c} \overline{M_{-1} F}=$ $F(\cdot / c)$. For a general function $h$ and positive $c$, we define also $M_{c} h$ to be $h(\cdot / c)$.

2.1. The Laplace characters. Let $\mathrm{D}^{k}$ be the differential operator defined by $\mathrm{D}^{k} h(x)=h^{(k)}(x)$ for any $k$ times differentiable function $h$. As usual, we set $\mathrm{D}^{0}$ to be the identity and $\mathrm{D}^{1}=\mathrm{D}$.

Recall that a linear differential operator with constant coefficients is a finite $\operatorname{sum} \sum_{0 \leqslant i \leqslant m} p_{i} \mathrm{D}^{i}$. This is a polynomial in $\mathrm{D}$, and the order of the operator is the degree of the polynomial. Thus, $p_{m}$ is nonzero if and only if the previous differential operator is of order $m$. We write $\mathbb{R}_{m}[\mathrm{D}]$ the set of all linear differential operators with constant 
coefficients and order at most $m$. It is naturally endowed with a vector space structure, corresponding to that of the polynomials. We will be mostly interested in a ring structure though. In $\mathbb{R}_{m}[\mathrm{D}]$, we define a composition as follows. If

$$
p=\sum_{0 \leqslant i \leqslant m} p_{i} \mathrm{D}^{i} \quad \text { and } \quad q=\sum_{0 \leqslant i \leqslant m} q_{i} \mathrm{D}^{i},
$$

are in $\mathbb{R}_{m}[\mathrm{D}]$, we set

$$
p \circ q=\sum_{0 \leqslant i \leqslant m}\left(\sum_{0 \leqslant j \leqslant i} p_{j} q_{i-j}\right) \mathrm{D}^{i} .
$$

In other words, we use for the ring structure of $\mathbb{R}_{m}[\mathrm{D}]$ that of the quotient ring of the polynomials in $\mathrm{D}$ modulo the ideal generated by $\mathrm{D}^{m+1}$.

In the following, we use the notation $\mu_{F, k}$ for the moment of order $k$ of a distribution function $F$, that is $\int x^{k} \mathrm{~d} F(x)$. In particular, $\mu_{F, 0}$ is 1 .

Definition. Let $F$ be a distribution function and $m$ be an integer such that $F$ has a finite $m$-th absolute moment. The $m$-th Laplace character of $F$ is the element of $\mathbb{R}_{m}[\mathrm{D}]$ defined by

$$
L_{F, m}=\sum_{0 \leqslant k \leqslant m} \frac{(-1)^{k}}{k !} \mu_{F, k} \mathrm{D}^{k}
$$

When $m$ is clear from the context, we may simply write $L_{F}$. Observe that $L_{\delta_{0}}$ is the identity. The origin of the term 'Laplace character' will be explained after our next proposition.

Proposition 2.1.1. If $F$ and $G$ are two distribution functions with finite absolute moment of order $m$, then $L_{F, m} \circ L_{G, m}=L_{F \star G, m}$.

Proof. The binomial formula shows that

$$
\sum_{0 \leqslant i \leqslant k} \frac{\mu_{F, i}}{i !} \frac{\mu_{G, k-i}}{(k-i) !}=\frac{\mu_{F \star G, k}}{k !}
$$

The conclusion follows in an easy way from the definition of the composition. 
The previous proposition asserts that the Laplace characters are morphisms of semigroups. Since the maps $F \mapsto \mu_{F, k}$ are linear, the Laplace characters are representations of convolution algebras in $\mathbb{R}_{m}[\mathrm{D}]$. One may wonder if other representations exist, that depend on the distribution through some other set of characteristics, say for instance, moments of fractional order or quantiles. In fact, the answer is negative. By a recent result of Mattner (2004), the mapping of a distribution with $m$ moments to its first $m$ cumulants is universal among all continuous homomorphisms of the convolution algebra of distributions with $m$ moments into Hausdorff topological groups. This implies in particular that one can express the Laplace characters in terms of cumulants.

We can now explain our choice of the name 'Laplace character'. Formally, $L_{F, m}$ is the $m$-th Taylor polynomial of the Laplace transform of $F$ where we substitute the operator $\mathrm{D}$ for the variable. Hence the 'Laplace' part of the name. Equivalently, we could define $L_{K, m}$ to be $E e^{-Y \mathrm{D}}$ in $\mathbb{R}_{m}[\mathrm{D}]$, where $Y$ has distribution function $K-$ note that with respect to Mattner's (2004) result, it is clear from this definition that the Laplace characters can be expressed in terms of cumulants. Moreover, Proposition 2.1.1 shows that the Laplace character obeys a property very similar to the Laplace transform, namely that this trivializes the convolution into a product of polynomials (modulo an ideal here). This property is very similar to that defining the characters of a group, hence the name. There is an other reason for the use of the word 'character' which has to do with the fact that $L_{F, m}$ can be viewed as a formal sum of differential operators, which reminded us about the Chern character in algebraic topology. And we also observe that the representation of convolution algebra is pretty much the same as the representation of modules realized by the Chern character, our module being over the field of real numbers.

One needs to be careful with one subtlety, namely that our composition in $\mathbb{R}_{m}[\mathrm{D}]$ is not the usual composition of operators acting on functions. If $T_{1}$ and $T_{2}$ are two operators, we write $T_{2} T_{1}$ for their usual composition, that is $\left(T_{2} T_{1}\right) h=T_{2}\left(T_{1} h\right)$ for any smooth function $h$. We then see that if $i+j>m$, we have $\mathrm{D}^{i} \mathrm{D}^{j}=\mathrm{D}^{i+j}$ while $\mathrm{D}^{i} \circ \mathrm{D}^{j}=0$. However, $\mathrm{D}^{i} \mathrm{D}^{j}$ is $\mathrm{D}^{i} \circ \mathrm{D}^{j}$ modulo an operator in the ideal generated by $\mathrm{D}^{m+1}$. This is in fact important (see subsection 5.2 ). We could avoid this subtlety by defining operators acting on an infinite sequence of tuples of the form $\left(\bar{F}_{i}, \bar{F}_{i}^{(1)}, \ldots, \bar{F}_{i}^{(m)}\right), i \geqslant 1$. It 
is really a matter of taste, and we feel that the framework proposed here is somewhat more convenient for our purposes.

For computational purposes, it is of interest to note that operators in $\left(\mathbb{R}_{m}[\mathrm{D}], \circ\right)$ which are not in the ideal generated by $\mathrm{D}$ - in other words, those which, as a polynomial in $\mathrm{D}$, have a nonzero constant term - can be inverted. In particular, the Laplace characters can be inverted. To write an explicit formula for the inverse, recall that an ordered partition of length $k$ of an integer $n$ is a $k$-tuple of positive integers $p=\left(p_{1}, \ldots, p_{k}\right)$ such that $p_{1} \geqslant p_{2} \geqslant \cdots \geqslant p_{k}>0$ and $p_{1}+\cdots+p_{k}=n$. For such partition of length $k$, it is convenient for what follows to agree on the notation $p_{i}=0$ for all $i$ larger than $k$. We write $\mathcal{P}(m, n)$ for the set of all ordered partitions of length at most $m$ of $n$. For such partition $p$, we write $\Delta p$ for $\left(p_{1}-p_{2}, p_{2}-p_{3}, \ldots, p_{m}-p_{m+1}\right)$. Also for a tuple $k=\left(k_{1}, \ldots, k_{m}\right)$ and an integer $q$ we write $\left(\begin{array}{l}q \\ k\end{array}\right)=\left(\begin{array}{c}q \\ k_{1} \ldots k_{m}\end{array}\right)$ the multinomial type coefficient $q ! /\left(k_{1} ! \cdots k_{m} !\right)$.

Proposition 2.1.2. In $\mathbb{R}_{m}[\mathrm{D}]$, we have the inversion formula

$$
L_{F, m}^{-1}=\sum_{0 \leqslant n \leqslant m}\left(\sum_{p \in \mathcal{P}(m, n)}(-1)^{n+p_{1}}\left(\begin{array}{c}
p_{1} \\
\Delta p
\end{array}\right) \prod_{1 \leqslant k \leqslant m}\left(\frac{\mu_{F, k}}{k !}\right)^{(\Delta p)_{k}}\right) \mathrm{D}^{n} .
$$

Proof. In this proof we write $i=\left(i_{1}, \ldots, i_{m}\right)$ a generic tuple of nonnegative integers, and $|i|=i_{1}+\cdots+i_{m}$. In the space of power series in $t$,

$$
\begin{aligned}
\left(1+\sum_{1 \leqslant k \leqslant m}\right. & \left.\frac{(-1)^{k}}{k !} \mu_{F, k} t^{k}\right)^{-1} \\
& =\sum_{j \geqslant 0}(-1)^{j}\left(\sum_{1 \leqslant k \leqslant m} \frac{(-1)^{k}}{k !} \mu_{F, k} t^{k}\right)^{j} \\
& =\sum_{j \geqslant 0}(-1)^{j} \sum_{|i|=j}\left(\begin{array}{l}
j \\
i
\end{array}\right) \prod_{1 \leqslant k \leqslant m}\left(\frac{(-1)^{k}}{k !} \mu_{F, k} t^{k}\right)^{i_{k}} .
\end{aligned}
$$

Consequently, in $\mathbb{R}_{m}[\mathrm{D}]$, the inverse of $L_{F, m}$ is

$$
\sum_{0 \leqslant j \leqslant m}(-1)^{j} \sum_{i}\left(\begin{array}{l}
j \\
i
\end{array}\right)\left(\prod_{1 \leqslant k \leqslant m}\left(\frac{(-1)^{k}}{k !} \mu_{F, k}\right)^{i_{k}}\right) \mathrm{D}^{i_{1}+2 i_{2}+\cdots+m i_{m}}
$$


where the sum over $i$ is over all tuples $\left(i_{1}, \ldots, i_{m}\right)$ such that $|i|=j$ and $i_{1}+2 i_{2}+\cdots+m i_{m} \leqslant m$. Set $p_{m+1}=0$ and $p_{l}=i_{l}+p_{l+1}$ for $l=m, m-1, \ldots, 1$. Thus, $p_{m}=i_{m}, p_{m-1}=i_{m}+i_{m-1}, \ldots$, $p_{1}=i_{m}+\cdots+i_{1}$. Set $p=\left(p_{1}, \ldots, p_{m}\right)$. We see that $\sum_{1 \leqslant k \leqslant m} k i_{k}=n$ if and only if $p$ is an ordered partition of $n$, and the correspondance between $p$ and $i$ is one-to-one. Moreover, $|i|=p_{1}$. Therefore

$$
L_{F, m}^{-1}=\sum_{n \leqslant m}\left(\sum_{p \in \mathcal{P}(m, n)}(-1)^{p_{1}}\left(\begin{array}{c}
p_{1} \\
\Delta p
\end{array}\right) \prod_{1 \leqslant k \leqslant m}\left(\frac{(-1)^{k}}{k !} \mu_{F, k}\right)^{(\Delta p)_{k}}\right) \mathrm{D}^{n},
$$

which is the inversion formula.

To use Proposition 2.1.2, one may need to generate all the partitions of a given integer. We refer to Nijenhuis and Wilf (1978) or Stanton and White (1986) for algorithms related to that matter.

Our next result is an other inversion formula for the Laplace characters. It is not as explicit as the previous one, because it is written in the ring $\left(\mathbb{R}_{m}[\mathrm{D}], \circ\right)$. However, we will make use of it. To write this inversion formula, for any differential operator $T$ in $\left(\mathbb{R}_{m}[\mathrm{D}], \circ\right)$ and any nonnegative integer $k$, we define $T^{\circ k}$ inductively by $T^{\circ 0}=\operatorname{Id}$ and $T^{\circ k}=T \circ T^{\circ(k-1)}$.

Proposition 2.1.3. In $\left(\mathbb{R}_{m}[\mathrm{D}], \circ\right)$, we have the inversion formula

$$
L_{F, m}^{-1}=\sum_{0 \leqslant k \leqslant m}\left(\mathrm{Id}-L_{F, m}\right)^{\circ k}=\sum_{0 \leqslant j \leqslant m}(-1)^{j} L_{F, m}^{\circ j} \sum_{j \leqslant k \leqslant m}\left(\begin{array}{l}
k \\
j
\end{array}\right) .
$$

Proof. Since $L_{F, m}-\mathrm{Id}$ is in the ideal generated by $\mathrm{D}$, it is nilpotent of nullity at most $m+1$ in $\left(\mathbb{R}_{m}[D], \circ\right)$. Consequently,

$$
L_{F, m}^{-1}=\left(\operatorname{Id}-\left(\operatorname{Id}-L_{F, m}\right)\right)^{-1}=\sum_{0 \leqslant k \leqslant m}\left(\operatorname{Id}-L_{F, m}\right)^{\circ k} .
$$

Then, the second equality follows from the binomial formula

$$
\left(\operatorname{Id}-L_{F, m}\right)^{\circ k}=\sum_{0 \leqslant j \leqslant k}\left(\begin{array}{l}
k \\
j
\end{array}\right)(-1)^{j} L_{F, m}^{\circ j} .
$$

It is also clear that there are other ways of thinking of the Laplace characters and their inverses that can be useful in applications. For 
instance we can think of $L_{F, m}$ as the $m$-th Taylor polynomial of the Laplace transform $\mathcal{L}_{F}$ (if it exists) of $F$ evaluated at D. Then $L_{F, m}^{-1}$ is simply obtained by taking the $m$-th Taylor polynomial of $1 / \mathcal{L}_{F}$ and evaluating it in the variable D.

There are other algebraic operations on Laplace characters which are of interest. For instance, we will use the Mellin-Stieltjes convolution between two distribution functions $F$ and $G$ on the nonnegative half line. It is writen $F \stackrel{\mathrm{M}}{*} G$, and defined by

$$
F \stackrel{\mathrm{M}}{*} G(t)=\int_{0}^{\infty} F(t / x) \mathrm{d} G(x)
$$

If $X$ and $Y$ are two independent random variables with respective distributions $F$ and $G$, then $F \stackrel{\text { M }}{*} G$ is the distribution of the product $X Y$. Since $E(X Y)^{j}=E X^{j} E Y^{j}$, the Laplace character of $F \stackrel{\text { M }}{*} G$ is obtained by multiplying the Laplace characters of $F$ and $G$ coefficient-wise, that is

$$
L_{F \stackrel{M}{*} G, m}=\sum_{0 \leqslant j \leqslant m} \frac{(-1)^{j}}{j !} \mu_{F, j} \mu_{G, j} \mathrm{D}^{j} .
$$

Yet, another algebraic operation that one could consider, and which we will not use in this paper, is that of differentiating formally the Laplace characters as polynomials in D. Assuming that $\mu_{F, k}$ exists and does not vanish, writing $\mathrm{d} G(x)=\mu_{F, k}^{-1} x^{k} \mathrm{~d} F(x)$, we see that $L_{G, m}$ is $(-1)^{k} / \mu_{F, k}$ times the $k$-th derivative with respect to the variable $\mathrm{D}$ of $L_{F, m+k}$.

Similarly, if $F$ is a distribution on the nonnegative half line and $G(t)=\mu_{F, 1}^{-1} \int_{0}^{t} \bar{F}(x) \mathrm{d} x$, then

$$
L_{G, m}=-\mu_{F, 1}^{-1}\left(L_{F, m+1}-\mathrm{Id}\right) / \mathrm{D} .
$$

The interest of such algebraic formulas is to produce a form of operational calculus to derive tail expansions. This will be clear in section 3 .

We conclude this subsection with two lemmas. The first one is related to Proposition 2.1.1 while the second one describes the behavior of the Laplace characters under some multiplications. 
Lemma 2.1.4. If $H$ and $K$ are two distribution functions with finite $m$-th absolute moments, then

$$
L_{K \star H, m}=\sum_{0 \leqslant j \leqslant m} \frac{(-1)^{j}}{j !} \mu_{H, j} L_{K, m-j} \mathrm{D}^{j} .
$$

Proof. The right hand side of the equality is

$$
\sum_{0 \leqslant j \leqslant m} \frac{(-1)^{j}}{j !} \mu_{H, j} \sum_{0 \leqslant l \leqslant m-j} \frac{(-1)^{l}}{l !} \mu_{K, l} \mathrm{D}^{j+l} .
$$

Setting $s=l+j$, it is

$$
\begin{aligned}
\sum_{s, j \geqslant 0} \mathbb{I}\{j \leqslant s \leqslant m\} \frac{(-1)^{s}}{s !}\left(\begin{array}{l}
s \\
j
\end{array}\right) \mu_{H, j} \mu_{K, s-j} \mathrm{D}^{s} \\
=\sum_{0 \leqslant s \leqslant m} \frac{(-1)^{s}}{s !} \mu_{K \star H, s} \mathrm{D}^{s}
\end{aligned}
$$

which gives the conclusion.

Lemma 2.1.5. If $K$ is a distribution function whose $m$-th moment is finite, then

$$
L_{M_{\lambda} K, m} M_{\lambda}=M_{\lambda} L_{K, m}
$$

Proof. It follows from the equalities

$$
\begin{aligned}
L_{M_{\lambda} K, m}\left(M_{\lambda} h\right) & =\sum_{0 \leqslant j \leqslant m} \frac{(-1)^{j}}{j !} \mu_{M_{\lambda} K, j} \mathrm{D}^{j}\left(M_{\lambda} h\right) \\
& =\sum_{0 \leqslant j \leqslant m} \frac{(-1)^{j}}{j !} \lambda^{j} \mu_{K, j} \lambda^{-j} M_{\lambda} \mathrm{D}^{j} h,
\end{aligned}
$$

and the equality of the last term with $M_{\lambda} L_{K, m} h$.

2.2. Smoothly varying functions of finite order. Among the regularly varying functions, the normalized ones will be of importance to us. Following Bingham, Goldie and Teugels (1989, §1.3.2), we say that a function $g$ defined on $[a, \infty)$ is a normalized regularly varying function with index $\rho$ if it has the representation

$$
g(t)=t^{\rho} c \exp \int_{a}^{t} \frac{\epsilon(u)}{u} \mathrm{~d} u,
$$


where $\epsilon(\cdot)$ is a function converging to 0 at infinity.

For any real number $x$ and any positive integer $k$, we write $(x)_{k}$ for $x(x-1) \cdots(x-k+1)$. We also set $(x)_{0}=1$. If $h$ is a function, $h^{(k)}$ is the $k$-th derivative of $h$ if it exists, with the usual convention $h^{(0)}=h$.

Recall that a function $h$ defined in some neighborhood of infinity is smoothly varying with index $-\alpha$ if it is ultimately infinitely differentiable and

$$
\lim _{t \rightarrow \infty} t^{k} h^{(k)}(t) / h(t)=(-\alpha)_{k}
$$

for every integer $k$ (see Bingham, Goldie and Teugels, 1989, §1.8). The set of all smoothly varying functions with index $-\alpha$ is written $S R_{-\alpha}$. The set of smoothly varying functions of a given finite order which we will define contains $S R_{-\alpha}$ and can be thought of as a Sobolev space in the framework of regular variation. Before proceeding, it is useful to remark that for $k=1$ relation (2.2.2) forces $h$ to be normalized regularly varying of index $-\alpha$ (see Bingham, Goldie and Teugels, 1989, §1.8). This and (2.2.2) forces $h^{(k)}$ to be regularly varying of index $-\alpha-k$.

Definition. Let $m$ be a positive integer. A function $h$ is smoothly varying of index $-\alpha$ and order $m$ if it is ultimately $m$-times continuously differentiable and $h^{(m)}$ is regularly varying of index $-\alpha-m$. We write $S R_{-\alpha, m}$ for the set of all such functions.

From Karamata's theorem, we see that a function $h$ in $S R_{-\alpha, m}$ satisfies (2.2.2) for any $0 \leqslant k \leqslant m$. Note that if $m_{1}$ is at most $m_{2}$ then $S R_{-\alpha, m_{2}}$ is included in $S R_{-\alpha, m_{1}}$.

Also, if $h$ belongs to $S R_{-\alpha, m}$, then all $h^{(k)}$ 's for $k=0,1, \ldots, m-1$ are normalized regularly varying. Consequently, for any such $k$, the function $t \mapsto t^{\sigma}\left|h^{(k)}(t)\right|$ is ultimately decreasing (respectively increasing) when $\sigma<\alpha+k$ (respectively $\sigma>\alpha+k$ ) - see Bingham, Goldie and Teugels, 1989, Theorem 1.5.5.

To define $S R_{-\alpha, s}$ for a real number $s$, we write $\delta_{x}$ for the point mass at $x$. Thus if $h$ is a function, $\delta_{x} h=\int h \mathrm{~d} \delta_{x}=h(x)$. We then define the operator

$$
\Delta_{t, x}^{r}=\operatorname{sign}(x) \frac{\delta_{t(1-x)}-\delta_{t}}{|x|^{r} \delta_{t}}
$$


In other words, for any function $g$ we set

$$
\Delta_{t, x}^{r} g=\operatorname{sign}(x) \frac{g(t(1-x))-g(t)}{|x|^{r} g(t)} .
$$

Definition. Let $s$ be a positive real number. Write $s=m+r$ where $m$ is the integer part of $s$ and $r$ is in $[0,1)$. A function $h$ is smoothly varying of index $-\alpha$ and order $s$ if it belongs to $S R_{-\alpha, m}$ and

$$
\lim _{\delta \rightarrow 0} \limsup _{t \rightarrow \infty} \sup _{0<|x| \leqslant \delta}\left|\Delta_{t, x}^{r} h^{(m)}\right|=0 .
$$

Note that $m=m+0$, and that if $h$ belongs to $S R_{-\alpha, m}$ then (2.2.3) holds for $r=0$ thanks to the uniform convergence on compact subsets of $(0, \infty)$ theorem (Bingham, Goldie, Teugels,1989, Theorem 1.2.1)

When $s$ is smaller than 1 , the set $S R_{-\alpha, s}$ is closely related to that of all the functions satisfying the Lipschitz condition $\left[\mathrm{D}_{s}\right]$ of Borovkov and Borovkov (2002), while for positive $m$ it is related to their condition $\left[\mathrm{D}_{m}\right]$.

Our next result shows that these spaces are nested.

Proposition 2.2.1. If $r$ is at most $s$, then $S R_{-\alpha, s} \subset S R_{-\alpha, r}$.

Proof. If $r$ or $s$ is an integer, the result is obvious, hence we assume that both are not integers. Write $r=m+\rho$ and $s=n+\sigma$ with $m$ and $n$ integers and $\rho, \sigma$ positive and less than 1 . Let $h$ be a function in $S R_{-\alpha, s}$. If $n$ is at most $r$, then $m$ and $n$ are equal, and $\rho$ is less than $\sigma$. It is then plain that $h$ is in $S R_{-\alpha, r}$ since the function $|x|^{\sigma-\rho}$ is bounded in any neighborhood of the origin.

Assume that $n$ is larger than $r$. Then $n$ is at least $m+1$ and

$$
\lim _{t \rightarrow \infty} t^{m+1} h^{(m+1)}(t) / h(t)=(-\alpha)_{m+1} .
$$

Since $m+1$ is at least 1 , the function $h^{(m+1)}$ is regularly varying of index $-\alpha-m-1$. We write

$$
h^{(m)}(t(1-x))-h^{(m)}(t)=-\int_{1-x}^{1} t h^{(m+1)}(t u) \mathrm{d} u
$$

to obtain

$$
\left|\Delta_{t, x}^{\rho} h^{(m)}\right| \leqslant|x|^{1-\rho} \sup _{1-x \leqslant u \leqslant 1}\left|t h^{(m+1)}(t u) / h^{(m)}(t)\right| .
$$


Using the uniform convergence Theorem (Bingham, Goldie and Teugels, 1989, Theorem 1.2.1) and that $\rho$ is less than 1 , we conclude that

$$
\lim _{\delta \rightarrow 0} \limsup _{t \rightarrow \infty} \sup _{0<|x| \leqslant \delta}\left|\Delta_{t, x}^{\rho} h^{(m)}\right|=0,
$$

which shows that $h$ belongs to $S R_{-\alpha, r}$.

Before going further, let us make a digression about condition (2.2.3). One may wonder about the analogous condition when $r$ is 1. In fact, as we will explicate next, the condition (2.2.3) with $r=1$ has bearing on several issues, e.g. understanding the spaces $S R_{-\alpha, \omega}$, the monotone density theorem which is a classical result in the theory of regular variation, the class of asymptotically smooth functions introduced in Barbe and McCormick (2005) as well as the conditions [D] in Borovkov and Borovkov (2003).

For clarity of the arguments, if a function $g$ is differentiable, then

$$
\lim _{x \rightarrow 0} \Delta_{t, x}^{1} g=-t g^{\prime}(t) / g(t) .
$$

Therefore, if $\beta$ is a positive number and if $g^{\prime}$ is regularly varying with index $-\beta-1$,

$$
\lim _{t \rightarrow \infty} \lim _{x \rightarrow 0} \Delta_{t, x}^{1} g=\beta .
$$

For $r=1$, it is then natural to introduce the condition

$$
\lim _{\delta \rightarrow 0} \limsup _{t \rightarrow \infty} \sup _{0<|x|<\delta}\left|\Delta_{t, x}^{1} g-\beta\right|=0 .
$$

Proposition 2.2.2. The following are equivalent:

(i) condition (2.2.4).

(ii) $g$ is normalized regularly varying with index $-\beta$.

(iii) $g$ is ultimately absolutely continuous and a version of its RadonNykodym derivative is regularly varying with index $-\beta-1$.

Proof. We write $\dot{g}$ a Radon-Nykodym derivative of $g$ when it exists.

We start by proving that (iii) implies (ii). If (iii) holds, by Karamata theorem (see Bingham et al., 1989, Theorem 1.5.11), Id $\dot{g} / g \sim-\beta$ at infinity. Integrating $\dot{g} / g$ gives (ii).

Next, we prove that (ii) implies (iii). Consider the Karamata representation of $g$,

$$
g(t)=t^{-\beta} c \exp \int_{t_{0}}^{t} \frac{\epsilon(u)}{u} \mathrm{~d} u .
$$


Such a function is ultimately absolutely continuous and a RadonNykodym derivative is

$$
\dot{g}(t)=\frac{g(t)}{t}\left(-\beta+\frac{\epsilon(t)}{\beta}\right) .
$$

This implies (iii).

We now prove that (ii) implies (i). Again, the Karamata representation of $g$ yields for $t$ large enough and $x$ small enough,

$$
\Delta_{t, x}^{1} g=\frac{(1-x)^{-\beta}-1}{x}+(1-x)^{-\beta} \frac{1}{x}\left(\exp \int_{t}^{t(1-x)} \frac{\epsilon(u)}{u} \mathrm{~d} u-1\right) .
$$

Let $\eta$ be a positive number and let $t_{1}$ be large enough so that $g$ is absolutely continuous on $\left(t_{1}, \infty\right)$ and the absolute value of $\epsilon(\cdot)$ is at most $\eta$ on $\left(t_{1}, \infty\right)$. Then, for any $t$ and $t(1-x)$ more than $t_{1}$,

$$
-\eta|\log (1-x)| \leqslant \int_{t}^{t(1-x)} \frac{\epsilon(u)}{u} \mathrm{~d} u \leqslant \eta|\log (1-x)| .
$$

For $\delta$ small enough, this implies

$$
\limsup _{t \rightarrow \infty} \sup _{0<|x|<\delta}\left|\frac{1}{x}\left(\exp \int_{t}^{t(1-x)} \frac{\epsilon(u)}{u} \mathrm{~d} u-1\right)\right| \leqslant 2 \eta .
$$

On the other hand,

$$
\lim _{\delta \rightarrow 0} \limsup _{t \rightarrow \infty} \sup _{0<|x|<\delta}\left|\frac{(1-x)^{-\beta}-1}{x}-\beta\right|=0 .
$$

Therefore, (2.2.4) and (i) hold.

We conclude the proof of the Proposition by showing that (i) implies (ii). We first note that if (2.2.4) holds, then $g$ must be ultimately continuous; otherwise, the supremum in $x$ in (2.2.4) would be infinite for some arbitrary large $t$ 's, precluding (2.2.4) to hold. Also, $g$ does not vanish ultimately. So, without loss of generality, we assume that $g$ is ultimately positive. Let $\dot{g}_{U}$ and $\dot{g}_{L}$ be the upper and lower derivatives of $g$, that is

$$
\begin{aligned}
& \dot{g}_{U}(t)=\limsup _{\epsilon \rightarrow 0} \frac{g(t+\epsilon)-g(t)}{\epsilon}, \\
& \dot{g}_{L}(t)=\liminf _{\epsilon \rightarrow 0} \frac{g(t+\epsilon)-g(t)}{\epsilon} .
\end{aligned}
$$


Condition (2.2.4) implies that Id $\dot{g}_{U} / g$ and $\operatorname{Id} \dot{g}_{L} / g$ have limit $-\beta$ at infinity. Set $f(t)=t^{\beta} g(t)$. Since $g$ is ultimately continuous,

$$
\dot{f}_{U}(t)=\beta t^{\beta-1} g(t)+t^{\beta} \dot{g}_{U}(t),
$$

and an analogous relation holds for the lower derivative of $f$. This implies

$$
t \dot{f}_{U}(t) / f(t)=\beta+t \dot{g}_{U}(t) / g(t)
$$

has limit 0 at infinity, and similarly for the upper derivative. By Bojanic and Karamata (1963) — see Bingham et al. 1989, exercice 1.11.8 - and Theorem 1.5.5 in Bingham et al. (1989), this implies that $f$ is normalized slowly varying.

Note that Proposition 2.2.2 implies that if a function $h$ belongs to $S R_{-\alpha, m}$ and (2.2.4) holds with $h^{(m)}$ in place of $g$ and $\beta=\alpha+m$, then the existence of the derivative $h^{(m+1)}$ guarantees that $h$ belongs to $S R_{-\alpha, m+1}$. However, without assuming that $h^{(m+1)}$ exists, (2.2.4) would only give existence and regular variation of a Radon-Nykodym derivative of $h^{(m)}$.

The equivalence between (ii) and (iii) in Proposition 2.2.2 shows that the class of normalized regularly varying functions is a very natural one when dealing with regular variation of a function and its derivative.

It also follows from Proposition 2.2.2 that the class of asymptotically smooth distributions in Barbe and McCormick (2005) is in fact those which are normalized regularly varying; this was suggested to us by Jaap Geluk and prompted Proposition 2.2.2. It also shows that condition $\left[\mathrm{D}_{m}\right]$ of Borovkov and Borovkov (2003) relates to normalized regular variation.

Proposition 2.2.2 is also interesting with respect to the monotone density theorem (Bingham et al., 1989, Theorem 1.7.2). Indeed, this theorem asserts that if a regularly varying function $g$ with index $-\beta$ has ultimately monotone derivative $g^{\prime}$, then this derivative is regularly varying of index $-\beta-1$; and moreover, by Karamata's theorem, Id $g^{\prime} / g \sim-\beta$. This implies that $g$ is normalized regularly varying, and therefore satisfies (ii) of Proposition 2.2.2. In other words, the monotone density theorem can be viewed as a particular case of the implication of (iii) by (ii) in Proposition 2.2.2. This concludes our digression on condition (2.2.3) 
The importance of the classes $S R_{-\alpha, m}$ in this paper stems from the nice behavior of their functions with respect to differentiation, global Potter type bounds, and to Taylor formula used asymptotically. In order to elaborate on this assertion, recall first that Potter's bounds (see, Bingham, Goldie and Teugels, 1989, Theorem 1.5.6) assert that if $g$ is a function which is regularly varying with index $\rho$, then for any $A$ larger than 1 and any $\delta$ positive, there exists a $t_{0}$ such that for any $\lambda$ at least 1 and any $t$ more than $t_{0}$,

$$
A^{-1} \lambda^{\rho-\delta} \leqslant g(\lambda t) / g(t) \leqslant A \lambda^{\rho+\delta} .
$$

This standard Potter inequality already yields an upper bound on the decay of the scaled derivatives of smoothly varying functions of fixed order.

Lemma 2.2.3. Let $h$ be a smoothly varying function of index $-\alpha$ and order $s$ more than 1 . Let $\epsilon$ be a positive number. There exists $t_{1}$ such that for any $c$ positive at most 1 and any $t$ at least $t_{1}$ and any integer $k$ at most $s$,

$$
\left|\left(M_{c} h\right)^{(k)}(t)\right| \leqslant 2\left|(-\alpha)_{k}\right| c^{\alpha-\epsilon} t^{-k}|h(t)| .
$$

Proof. We have $\left(M_{c} h\right)^{(k)}(t)=c^{-k} h^{(k)}(t / c)$. Since $h$ is in $S R_{-\alpha, s}$ with $s$ at least $k$, there exists $t_{0}$ such that for any $t$ at least $t_{0}$,

$$
\left|t^{k} h^{(k)}(t) / h(t)\right| \leqslant \sqrt{2}\left|(-\alpha)_{k}\right|
$$

Since $c$ is positive and at most 1 , the inequality $t \geqslant t_{0}$ implies $t / c \geqslant t_{0}$. Therefore, for $t$ at least $t_{0}$,

$$
c^{-k}\left|h^{(k)}(t / c)\right| \leqslant \sqrt{2}\left|(-\alpha)_{k}\right| t^{-k}|h(t / c)| .
$$

Now, if $t$ is larger than some $t_{1}$ independent of $c$, Potter's bounds give $|h(t / c)| \leqslant \sqrt{2} c^{\alpha-\epsilon}|h(t)|$. This implies the result.

The following lemma shows that for normalized regularly varying functions, in particular for smoothly varying functions of fixed order at least 1 Potter's bounds can be improved by taking $A=1$. Although this may seem a minor feature, this improvement is essential for our purpose. 
Lemma 2.2.4. Let $g$ be a normalized regularly varying function with index $\rho$. Let $\delta$ be a positive number. There exists $t_{2}$ such that for any $\lambda$ larger than 1 and any $t$ at least $t_{2}$,

$$
\lambda^{\rho-\delta} \leqslant g(t \lambda) / g(t) \leqslant \lambda^{\rho+\delta} .
$$

Proof. Let $\epsilon(\cdot)$ be as in (2.2.1). Let $t_{2}$ be at least $a$ and such that $\sup _{t \geqslant t_{2}}|\epsilon(t)| \leqslant \delta$. Then,

$$
\frac{g(\lambda t)}{g(t)}=\lambda^{\rho} \exp \left(\int_{t}^{t \lambda} \frac{\epsilon(u)}{u} \mathrm{~d} u\right)\left\{\begin{array}{l}
\leqslant \lambda^{\rho} \exp \left(\delta \int_{t}^{t \lambda} u^{-1} \mathrm{~d} u\right) \\
\geqslant \lambda^{\rho} \exp \left(-\delta \int_{t}^{t \lambda} u^{-1} \mathrm{~d} u\right),
\end{array}\right.
$$

which yields the result.

To connect Taylor formula and asymptotic expansions, we set

$$
\bar{\Delta}_{\tau, \delta}^{q}(h)=\sup _{t \geqslant \tau} \sup _{0<|x| \leqslant \delta}\left|\Delta_{t, x}^{q} h\right| .
$$

Proposition 2.2.5. Let $r$ be in $[0,1]$. If $h$ is $m$ times differentiable, then for any positive $t$ and any $u$,

$$
\left|h(t+u)-\sum_{0 \leqslant j \leqslant m} \frac{u^{j}}{j !} h^{(j)}(t)\right| \leqslant \frac{|u|^{m+r}}{t^{r}} \frac{\left|h^{(m)}(t)\right|}{m !} \bar{\Delta}_{t,|u| / t}^{r}\left(h^{(m)}\right) .
$$

Proof. The Taylor-McLaurin formula yields

$$
h(t+u)=\sum_{0 \leqslant j \leqslant m} \frac{u^{j}}{j !} h^{(j)}(t)+\frac{u^{m}}{m !}\left(h^{(m)}\left(t+\theta_{t, u} u\right)-h^{(m)}(t)\right)
$$

with $\theta_{t, u}$ between 0 and 1 . The equality

$$
\left|h^{(m)}\left(t+\theta_{t, u} u\right)-h^{(m)}(t)\right|=\theta_{t, u}^{r} t^{-r}|u|^{r}\left|h^{(m)}(t)\right|\left|\Delta_{t,-\theta_{t, u} u / t}^{r} h^{(m)}\right|
$$

implies the result.

Observe that Lemma 2.2 .3 asserts that if $h$ is smoothly varying of order $s=m+r$, then $h^{(m)}(t)$ is of order $t^{-m} h(t)$. Thus, the remainder term in Proposition 2.2.5 is expected to be asymptotically 
rather small and certainly of smaller order of magnitude than any of the $h^{(j)}(t)$ involved in the inequality of the Proposition. In other words, for functions in $S R_{-\alpha, s}$ we can relate the local character of Taylor's formula to an asymptotic expansion of the translation $u \mapsto t+u$ acting on those functions. The Proposition implies that if $h$ is in $S R_{-\alpha, s}$, with $s$ larger than $m$, then $\sum_{0 \leqslant j \leqslant m}\left(u^{j} / j !\right) h^{(j)}(t)$ is an asymptotic expansion of $h(t+u)$ as $t$ tends to infinity. Using the translation $\tau_{x}$ defined on functions by $\tau_{x} h(t)=h(t+x)$, Proposition 2.2.5 asserts that $\tau_{u}$ is approximately the Laplace character $L_{\delta_{u}, m}$ when read on the tail of smoothly varying functions.

Before moving to the next section, we make a remark concerning the behavior of the operator $\Delta_{t, x}^{r}$ with respect to composition by differentiation and multiplications $M_{c}$. This remark does not have much intrinsic values, but we will need it during our proof.

Lemma 2.2.6. For any positive $c$, the equality $\Delta_{t, x}^{r} \mathrm{D}^{j} M_{c}=$ $\Delta_{t / c, x}^{r} \mathrm{D}^{j}$ holds.

Proof. We have

$$
\mathrm{D}^{j} M_{c} h(t)=\frac{\mathrm{d}^{j} h(t / c)}{\mathrm{d} t^{j}}=c^{-j} h^{(j)}(t / c)
$$

Therefore,

$$
\Delta_{t, x}^{r} \mathrm{D}^{j} M_{c} h=\frac{h^{(j)}((t / c)(1-x))-h^{(j)}(t / c)}{|x|^{r} h^{(j)}(t / c)}=\Delta_{t / c, x}^{r} \mathrm{D}^{j} h
$$

2.3. Asymptotic expansion for infinite convolution. To state our main result, recall that the $\ell_{p}$ norm of a sequence $c=\left(c_{i}\right)_{i \in \mathbb{Z}}$ is

$$
|c|_{p}=\left(\sum_{i \in \mathbb{Z}}\left|c_{i}\right|^{p}\right)^{1 / p}
$$

This defines a norm only if $p$ is at least 1 . Nevertheless, we will still use the same notation with the same meaning when $p$ is less than 1 . When $p$ is infinite, $|c|_{\infty}$ is simply the supremum of the $\left|c_{i}\right|$ 's. Given three nonnegative numbers $\alpha, \gamma$ and $\omega$, we define

$$
N_{\alpha, \gamma, \omega}(c)=|c|_{\gamma\left(\frac{\alpha}{\alpha+\omega} \wedge \frac{1}{2}\right)} \vee 2^{\alpha /(\alpha+\omega)}|c|_{\infty} .
$$


This may or may not be a norm, according to whether or not $\gamma \alpha /(\alpha+\omega)$ and $\gamma / 2$ are at least 1. For the values of $\gamma$ that we will use, that is $\gamma$ positive and less than 1 , this is not a norm.

The next theorem is the main result of the paper. It establishes a generalized expansion for some infinite weighted convolutions with respect to the asymptotic scale $\operatorname{Id}^{-i} \bar{F}, i \geqslant 0$. To state this result, we need further notation.

If $F, G, H$ are three distribution functions, with $H=F \star G$, we write $F=H \natural G$, the division of $H$ by $G$.

Note that if $c_{i}$ is negative, then the upper tail of $c_{i} X_{i}$ is driven by the lower tail of $X_{i}$. This induces some complications in stating a result for the tail of $\langle c, X\rangle$ because one needs stronger assumptions when the signs of the constants and the random variable can be arbirary. This explains the formulation of the next result. It does not cover all the possible variations, but seems to give what is needed in most applications. Other cases often may be obtained by simple changes in the proof.

For two functions $f$ and $g$, we write $f \asymp g$ to mean that the ratio $f / g$ is ultimately bounded away from 0 and infinity.

In the following theorem, we write $G_{c}$ for the distribution of $\sum_{i \in \mathbb{Z}} c_{i} X_{i}$. Note that $G_{c} \natural M_{c_{i}} F$ is the distribution of that infinite series with the $i$-th term removed.

Theorem 2.3.1. Let $\omega$ be at least 1. Let $F$ be a distribution function with $\bar{F}$ in $S R_{-\alpha, \omega}$. Let $m$ and $k$ be two integers such that $m$ is smaller than $\alpha$, and $m+k$ is smaller than $\omega$. Furthermore, if some $c_{i}$ 's are negative assume either that $\overline{M_{-1} F}$ is also in $S R_{-\alpha, \omega}$ and $\overline{M_{-1} F} \asymp \bar{F}$ or that $F$ vanishes in a neighborhood of $-\infty$.

Let $\gamma$ be a positive number less than $\omega-m-k$ and 1 . Then, there exists a function $\eta(\cdot)$ converging to 0 at infinity and a real number $t_{0}$ such that for any $t$ at least $t_{0}$, for any sequence $c$ with $N_{\alpha, \gamma, \omega}(c) \leqslant 1$,

$$
\left|\bar{G}_{c}^{(k)}(t)-\sum_{i \in \mathbb{Z}} L_{G_{c} \sharp M_{c_{i}} F, m}\left(\overline{M_{c_{i}} F}\right)^{(k)}(t)\right| \leqslant t^{-m-k} \bar{F}(t) \eta(t) .
$$

In particular for any sequence $c$ with $N_{\alpha, \gamma, \omega}(c)$ finite,

$$
\bar{G}_{c}^{(k)} \sim \sum_{i \in \mathbb{Z}} L_{G_{c} \sharp M_{c_{i}} F, m}\left(\overline{M_{c_{i}} F}\right)^{(k)} \quad\left(\operatorname{Id}^{-m-k} \bar{F}\right) .
$$


This theorem calls for several remarks.

Remark. By allowing a certain uniformity in the Potter type bounds and in the asymptotic smoothness assumption, a degree of uniformity in our result with respect to the underlying distribution $F$ can be achieved. However, we choose not to develop such a refinement since it would entail a greater level of technicality which may be distractive to the main aim of the paper.

Remark. When some $c_{i}$ 's are negative, the assumption $\bar{F} \asymp \overline{M_{-1} F}$ is not necessary. It is quite clear from the proof how to modify the statement if we assume that $\overline{M_{-1} F}$ is in some $S R_{-\beta, \omega^{\prime}}$. However, the statement is far nicer when either both tails are comparable, or when $F$ is supported by the nonnegative half line.

Remark. When the $c_{i}$ 's are nonnegative, one does not really need to assume $\overline{M_{-1} F}=O(\bar{F})$. Certainly, the result holds provided only that the $m$-th absolute moment of the distribution is finite. This technical point appears in Lemma 6.3.1.

Remark. If either $k$ or $m$ are positive and their sum is less than $\omega$, then $\omega$ is more than 1 , and the first sentence in Theorem 2.3.1 is not needed. Assuming that $\omega$ is at least 1 is only needed when both $k$ and $m$ vanish; it is assumed only to ensure that $\bar{F}$ and possibly $\overline{M_{-1} F}$ are normalized regularly varying.

Remark. The analoguous statement on the lower tail holds as well.

Remark. Since the Laplace characters are differential operators with constant coefficients, they commute with the derivative D. Consequently, Theorem 2.3.1 implies that for a certain class of distributions and sequences, taking limit with respect to the number of nonvanishing terms in the sequence $c$ and differentiation can be permuted in an asymptotic expansion. It is quite striking that this can be done with some uniformity with respect to a rather large class of sequences.

Remark. It will be clear in the sequel that the formulation of Theorem 2.3.1 is well suited for applications. One may still wonder if one can relax its assumptions on $F$. The example of first order autoregressive processes suggests that some refinements may be 
given, but not in a fundamental way. Indeed, consider the sequence $c_{i}=a^{i}$ for nonnegative integers $i$, and $c_{i}=0$ for negative $i$. Thus, $G_{c}$ is the distribution of $Y=\sum_{i \geqslant 0} a^{i} X_{i}$. For any integer $k$, set

$$
Z_{k, i}=\sum_{0 \leqslant j<k} a^{j} X_{k i+j}
$$

For fixed $k$, as $i$ runs through the nonnegative integers, these random variables are independent and equidistributed. Moreover, $Y=\sum_{i \geqslant 0} a^{k i} Z_{k, i}$. Hence, if the distribution function of $Z_{k, i}$ fulfills the assumptions of Theorem 2.3.1, one can obtain the tail expansion of $G_{c}$, even though the distribution of $X_{i}$ may not satisfy the assumptions of Theorem 2.3.1. This may happen for instance if the distribution of $X_{i}$ is a mixture containing point masses. Note however that the tail expansion will be expressed in term of the distribution of $Z_{k, i}$ and not of $X_{i}$. One would then need to relate the derivative of the distribution function of $Z_{k, i}$ to that of $X_{i}$. In the problem at hand, if $Z_{k, i}$ has a density for $k$ large enough, then by increasing $k$, one can assume that this density is smooth. However, if no matter how large $k$ is $Z_{k, i}$ does not have a density, then $Y$ may still have one. As mentioned in the introduction, Solomyak's (1995) work is a cruel reminder on how difficult it is to study the marginal distribution of such a basic time series model.

For more general linear processes, one could imagine to use similar blocking techniques. One would then need to extend Theorem 2.3.1 in replacing the $M_{c_{i}} F$ 's by some more general $F_{i}$ 's, each $F_{i}$ being in $S R_{-\alpha, \omega}$. Formally, the expression is obvious to obtain. A proof along the line of Theorem 2.3.1 is possible under some assumptions on the $F_{i}$ 's. However, it is not clear that such a refinement presents much interest in applications. There is in fact a great similarity with the central limit theorem for density, where one does not need the summands to have a density, but the characteristic function to be in some $L^{r}$ space with some $r$ at least 1 (see Feller, 1971, $\S$ XV.5). Yet, in most real life applications - if not all — the local central limit theorem is applied when the summands have a well behaved density. The same seems true about the conditions of Theorem 2.3.1.

\section{Implementing the expansion.}

In this section we illustrate Theorem 2.3.1 and its implementation through examples. In the first subsection we discuss the number of terms in the expansion given by Theorem 2.3.1. In the second 
subsection, we explain how for standard statistical distributions, matrix identification of the differential and multiplication operators provides a computationally efficient way to derive tail expansions. In the third subsection, we discuss an open problem related to second order formula for weighted convolutions under the assumption of regular variation with remainder.

In what follows, if the sequence $c$ is nonnegative, we write $C_{p}$ for the series $\sum_{i \in \mathbb{Z}} c_{i}^{p}$. When $C_{p+q}$ as well as $C_{p}$ and $C_{q}$ are finite, we write $C_{p ; q}$ for $C_{p+q}-C_{p} C_{q}$.

3.1. How many terms are in the expansion? Let us consider the expansion given in Theorem 2.3.1 for the tail of the distribution function, that is when $k$ vanishes. It asserts

$$
\bar{G}_{c}=\sum_{i \in \mathbb{Z}} L_{G_{c} \sharp M_{c_{i}} F, m} \overline{M_{c_{i}} F}+o\left(\operatorname{Id}^{-m} \bar{F}\right) .
$$

It seems that this formula provides an $m+1$-term expansion. However, one should remember that the number of terms in an expansion depends crucially on the asymptotic scale chosen. In the case at hand, we will show that, for natural scales, this formula may give more or less than $m$ terms. We suspect that this fact explains the failure of previous purely analytical attempts to find only a two terms expansion in the general infinite order case. The algebraic flavor of the Laplace characters will be developed further in the next subsection, and will be the key to efficiently derive asymptotic expansions on specific distributions.

When $m$ is 1 , formula (3.1.1) is

$$
\bar{G}_{c}=\sum_{i \in \mathbb{Z}}\left(\overline{M_{c_{i}} F}-\mu_{G \natural M_{c_{i}} F, 1} \overline{M_{c_{i}} F^{\prime}}\right)+o\left(\operatorname{Id}^{-1} \bar{F}\right) .
$$

For simplicity of the discussion, assume that the $c_{i}$ 's are nonnegative, and let us ask: how many terms does (3.1.2) provide?

A naive answer is 2 , for the first term is $\sum_{i \in \mathbb{Z}} \overline{M_{c_{i}} F}$, while the second one is $-\sum_{i \in \mathbb{Z}} \mu_{G \natural M_{c_{i}} F, 1} \overline{M_{c_{i}} F^{\prime}}$. The following four examples show that the truth is more complex.

Example 1. Take $\bar{F}(t)=t^{-\alpha}(1+1 / \log t)$ for $t$ large enough. Then

$$
\begin{aligned}
\overline{M_{c} F}(t) & =\frac{c^{\alpha}}{t^{\alpha}}\left(1+\frac{1}{\log t-\log c}\right) \\
& =\frac{c^{\alpha}}{t^{\alpha}}+\frac{c^{\alpha}}{t^{\alpha} \log t}+\frac{c^{\alpha}}{t^{\alpha} \log t} \frac{\log c}{\log t-\log c} .
\end{aligned}
$$


Moreover, $\overline{M_{c} F^{\prime}}(t) \sim-\alpha c^{\alpha} / t^{\alpha+1}$ as $t$ tends to infinity. Applying formula (3.1.2), we obtain

$$
\bar{G}_{c}(t)=\frac{C_{\alpha}}{t^{\alpha}}+\frac{C_{\alpha}}{t^{\alpha} \log t}(1+o(1)) .
$$

The interesting point is that this two terms expansion is given by the sum $\sum_{i \in \mathbb{Z}} \overline{M_{c_{i}} F}$; it does not involve the term $\sum_{i \in \mathbb{Z}} \mu_{G \sharp M_{c_{i}} F, 1} \overline{M_{c_{i}} F^{\prime}}$.

Example 2. Take $\bar{F}(t)=t^{-\alpha}+t^{-\alpha-2}$ for $t$ large enough, and assume that $F$ is supported inside the nonnegative half line. This last assumption ensures that $\mu_{F, 1}$ does not vanish. Now,

$$
\bar{F}^{\prime}(t)=-\alpha t^{-\alpha-1}-(\alpha+2) t^{-\alpha-3} .
$$

Consequently, (3.1.2) yields

$$
\begin{aligned}
\bar{G}_{c}(t) & =C_{\alpha} t^{-\alpha}-\alpha t^{-\alpha-1} \sum_{i \in \mathbb{Z}}\left(C_{1}-c_{i}\right) c_{i}^{\alpha} \mu_{F, 1}+o\left(t^{-\alpha-1}\right) \\
& =C_{\alpha} t^{-\alpha}+\alpha C_{\alpha ; 1} \mu_{F, 1} t^{-\alpha-1}+o\left(t^{-\alpha-1}\right) .
\end{aligned}
$$

Therefore, if $C_{1} C_{\alpha} \neq C_{\alpha+1}$, the first term is given by a contribution from $\sum_{i \in \mathbb{Z}} \overline{M_{c_{i}} F}$, while the second one comes from $\sum_{i \in \mathbb{Z}} \bar{M}_{c_{i}} F^{\prime}$.

Example 3. Take $\bar{F}(t)=t^{-\alpha}-t^{-\alpha-3}$ for large $t$, and assume now that $F$ is symmetric. Hence $\mu_{F, 1}$ vanishes. Formula (3.1.2) yields

$$
\bar{G}_{c}(t)=C_{\alpha} t^{-\alpha}+o\left(t^{-\alpha-1}\right) .
$$

In some sense, the formula fails to give a two terms expansion. So we consider formula (3.1.1) when $m$ is 2 . Since $\mu_{F, 1}$ vanishes, it yields

$$
\bar{G}_{c}(t)=\sum_{i \in \mathbb{Z}} \overline{M_{c_{i}} F}(t)+\frac{1}{2} \sum_{i \in \mathbb{Z}} \mu_{G \sharp M_{c_{i}} F, 2}{\overline{M_{c_{i}} F}}^{(2)}(t)+o\left(t^{-\alpha-2}\right) .
$$

Also, since $\mu_{F, 1}$ is zero,

$$
\mu_{G \natural M_{c_{i}} F, 2}=E\left(\sum_{\substack{j \in \mathbb{Z} \\ j \neq i}} c_{j} X_{j}\right)^{2}=\left(C_{2}-c_{i}^{2}\right) \mu_{F, 2} .
$$

Consequently,

$$
\begin{aligned}
\bar{G}_{c}(t) & =C_{\alpha} t^{-\alpha}+\frac{1}{2} \sum_{i \in \mathbb{Z}}\left(C_{2}-c_{i}^{2}\right) \mu_{F, 2} \alpha(\alpha+1) c_{i}^{\alpha} t^{-\alpha-2}+o\left(t^{-\alpha-2}\right) \\
& =C_{\alpha} t^{-\alpha}-\frac{\alpha(\alpha+1)}{2} C_{\alpha ; 2} \mu_{F, 2} t^{-\alpha-2}+o\left(t^{-\alpha-2}\right) .
\end{aligned}
$$


We see that the second term in this formula comes in fact from the third term in (3.1.1). Notice that the second term in the expansion for $\bar{F}$ played no role.

Example 4. Let us modify example 2 in writing $\bar{F}(t)=t^{-\alpha}+a t^{-\alpha-2}$ for large $t$. And let us take $F$ to be symmetric. Formula (3.1.1) with $m$ equal 2 yields

$$
\begin{aligned}
& \begin{array}{r}
\bar{G}_{c}(t)=C_{\alpha} t^{-\alpha}+a C_{\alpha+2} t^{-\alpha-2}+\frac{\alpha(\alpha+1)}{2} \mu_{F, 2} \sum_{i \in \mathbb{Z}}\left(C_{2}-c_{i}^{2}\right) c_{i}^{\alpha} t^{-\alpha-2} \\
+o\left(t^{-\alpha-2}\right)
\end{array} \\
& =C_{\alpha} t^{-\alpha}+t^{-\alpha-2}\left(a C_{\alpha+2}-0.5 \alpha(\alpha+1) \mu_{F, 2} C_{\alpha ; 2}\right)+o\left(t^{-\alpha-2}\right) \text {. }
\end{aligned}
$$

For any fixed sequence $c=\left(c_{i}\right)_{i \in \mathbb{Z}}$ of nonnegative numbers, we can find $a$ such that

$$
a C_{\alpha+2}-0.5 \alpha(\alpha+1) \mu_{F, 2} C_{\alpha ; 2}=0
$$

In this case, we need to include at least one more term, taking at least $m=3$ in formula (3.1.1) if we want two nonzero terms.

A first point of these examples is that obtaining simply a two terms expansion formula is rather hopeless in general. Clearly, some tricky cancelation may occur, depending on the particular sequence $c$ considered as well as the coefficients in the expansion of $\bar{F}$.

A second point is that when some cancellations occur, we may need to add terms; at least if we can, since the condition $m$ less than $\alpha$ caps the number of terms we can obtain. In our examples, this was easy, but in other cases, this may involve far more complicated calculations. Hence, we need more effective ways to implement the expansion, and this will be discussed in the next subsection.

A third point is that it raises an interesting question: Is it possible that for any fixed $m$, as large as we want, can we find a distribution for which formula (3.1.1) provides only a 1 term expansion? We address this question in the rest of this subsection. The answer is yes, though it is not a generic case. To make this clear, let us examine the following statement.

Statement. Generically, (3.1.1) with $m$ equal 2 provides at least a two terms expansion, even if $\mu_{F, 1}$ vanishes. 
When $m$ is 2, the right hand side in (3.1.1), up to the $o(\cdot)$-term, is

$$
\sum_{i \in \mathbb{Z}} \overline{M_{c_{i}} F}-\sum_{i \in \mathbb{Z}} \mu_{G \sharp M_{c_{i}} F, 1} \overline{M_{c_{i}} F^{\prime}}+\frac{1}{2} \sum_{i \in \mathbb{Z}} \mu_{G \sharp M_{c_{i}} F, 2} \overline{M_{c_{i}} F^{\prime \prime}} .
$$

Let $\sigma_{F}^{2}$ be the variance of $F$. Since

$$
\begin{aligned}
& \mu_{G \natural M_{c_{i} F, 1}}=\left(C_{1}-c_{i}\right) \mu_{F, 1} \\
& \mu_{G \natural M_{c_{i}} F, 2}=\left(C_{2}-c_{i}^{2}\right) \sigma_{F}^{2}+\left(C_{1}-c_{i}\right)^{2} \mu_{F, 1}^{2}
\end{aligned}
$$

and $\overline{M_{c_{i}} F^{\prime \prime}}(t) \sim c_{i}^{\alpha} \bar{F}^{\prime \prime}(t)$, as $t$ tends to infinity, the formula reads

$$
\begin{aligned}
\sum_{i \in \mathbb{Z}} \overline{M_{c_{i}} F} & -\sum_{i \in \mathbb{Z}}\left(C_{1}-c_{i}\right) \mu_{F, 1} \overline{M_{c_{i}} F^{\prime}} \\
& -\frac{1}{2}\left(C_{\alpha ; 2} \sigma_{F}^{2}+\left(C_{1} C_{\alpha ; 1}-C_{\alpha+1 ; 1}\right) \mu_{F, 1}^{2}\right) \bar{F}^{\prime \prime}(t)+o\left(\bar{F}(t) / t^{2}\right) .
\end{aligned}
$$

Generically, there is no cancellation, and even if $\mu_{F, 1}$ vanishes, the term in $\bar{F}^{\prime \prime}(t)$ yields a nonzero term which (generically) does not cancel with $\sum_{i \in \mathbb{Z}} \overline{M_{c_{i}} F}$. So, the formula yields at least two terms.

The companion of the positive statement is as follows.

Statement. Consider the formula (3.1.1) for both $m$ and the sequence $c=\left(c_{i}\right)_{i \in \mathbb{Z}}$ fixed. Then there exists a distribution (depending on $m$ and the sequence $c$ ) for which the formula yields only a 1-term expansion.

We will prove this statement in the next section, once we have an effective way of calculating expansions.

\subsection{Practical implementation: from Laplace's characters to} linear algebra. The few examples that we gave in the previous subsection were calculated easily by hand. In more complicated cases as well as for theoretical reasons (for instance to prove the statement at the end of the previous subsection), we need more effective ways to derive expansions. In Barbe and McCormick (2005), we introduced an algebraic technique, a tail calculus, which allows one to derive asymptotic expansions for finite weighted convolutions of distribution functions such that $\bar{F}(t) \sim t^{-\alpha} P(1 / t)$ for some polynomial $P$. An extension to infinite order moving averages 
was carried out for this class in Barbe and McCormick (200?). Distributions for which this calculus is applicable include the Pareto, Student and the Generalized Pareto. However, it does not apply to other well used distributions in the heavy tail literature. So we will develop a tail calculus for those. It may be possible to derive an abstract tail calculus which covers many more examples. However, we have not been able to find a good formalism for that purpose. Consequently we will say few things on the general idea, and mostly develop two examples.

Generalities on tail calculus. Assume that we start with a distribution function $F$ such that $\bar{F}$ and $\overline{M_{-1} F}$ have asymptotic expansions in an asymptotic scale $e=\left(e_{i}\right)_{i \in I}$ say. That is, for some coefficients $p_{\bar{F}, i}$ and $p_{\overline{M_{-1} F}, i}$

$$
\bar{F} \sim \sum_{i \in I} p_{\bar{F}, i} e_{i} \quad \text { and } \quad \overline{M_{-1} F} \sim \sum_{i \in I} p_{\overline{M_{-1} F}, i} e_{i} .
$$

We write $p_{\bar{F}}$ and $p_{\overline{M_{-1} F}}$ for the vectors $\left(p_{\bar{F}, i}\right)_{i \in I}$ and $\left(p_{\overline{M_{-1} F}, i}\right)_{i \in I}$. Note that since $e$ is an asymptotic scale and both $F$ and $\bar{F}$ are nonnegative, the first nonzero term of $p_{F}$ and $p_{\overline{M_{-1} F}}$ is positive. Writing $|I|$ the cardinality of $I$, we can think of these expansions as a coding of the tails in the vector space $\mathbb{R}^{2|I|}$ via the map

$$
\rho: F \mapsto \rho(F)=\left(p_{\bar{F}}, p_{\overline{M_{-1} F}}\right) .
$$

The expansion in Theorem 2.3.1 involves multiplication by constants. Both the derivative $\mathrm{D}$ and the multiplication operators $M_{c}$ act componentwise on the scale $e$ by $\mathrm{D} e=\left(\mathrm{D} e_{i}\right)_{i \in I}$ and $M_{c} e=$ $\left(M_{c} e_{i}\right)_{i \in I}$. For every $t$, we can take linear combinations of the components of the vector $e(t)$ by left multiplying it by a matrix. For vector valued functions, we extend the expansion notation $\sim$ as acting componentwise. So, a vector valued function $f=\left(f_{1}, \ldots, f_{p}\right)$ has asymptotic expansion $g=\left(g_{1}, \ldots, g_{p}\right)$ if $f_{i} \sim g_{i}$ for each $i=1, \ldots, p$.

Definition. An asymptotic scale $e=\left(e_{i}\right)_{i \in I}$ is a -asymptotic scale if there exist matrices $\mathcal{D}$ and $\mathcal{M}_{c}$ such that $\mathrm{D} e \sim \mathcal{D}$ e and for any $c$ positive, $M_{c} e \sim \mathcal{M}_{c} e$.

In particular, in an asymptotic sense, a $\star$-asymptotic scale is closed under differentiation and multiplication of the variable by a positive constant. 
For a $\star$-asymptotic scale, the following diagram commutes.

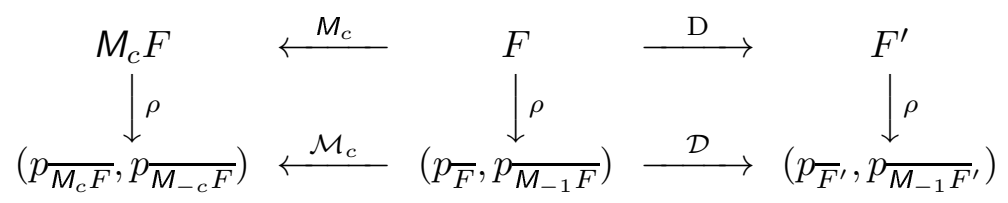

In particular, the identification of tails with the finite vector space $\mathbb{R}^{2|I|}$ implies that the operators $\mathcal{D}$ and $\mathcal{M}$ involved in the lower part of the diagram are simply matrices. This allows one to identify the Laplace character $L_{K, m}$ with the matrix

$$
\mathcal{L}_{K}=\sum_{0 \leqslant j \leqslant m} \frac{(-1)^{j}}{j !} \mu_{K, j} \mathcal{D}^{j}
$$

Note that this matrix depends on the order $m$ of the Laplace character as well as on the $\star$-asymptotic scale chosen. The notation does not show this dependence. Theorem 2.3.1 implies that the following diagram commutes.

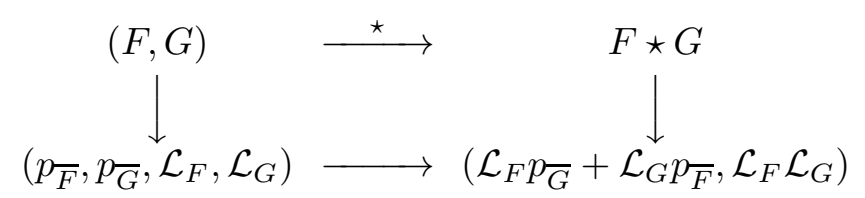

In particular, the map $F \mapsto \mathcal{L}_{F}$ is a linear representation of the convolution algebra, whose dimension is $|I|$.

Practically, the diagram says that on a $\star$-asymptotic scale, asymptotic expansions for weighted convolution can be done by manipulating finite dimensional vectors and matrices. This is the key to effective computation.

In particular, using the existence of inverse for the Laplace characters, the asymptotic expansion of Theorem 2.3.1 is given by

$$
\begin{aligned}
p_{\bar{G}_{c}^{(k)}}^{(k)} & =\sum_{i \in \mathbb{Z}} \mathcal{L}_{G_{c}} \mathcal{L}_{M_{c_{i}} F}^{-1} \mathcal{D}^{k} \mathcal{M}_{c_{i}}\left(\mathbb{I}\left\{c_{i}>0\right\} p_{\bar{F}}+\mathbb{I}\left\{c_{i}<0\right\} p_{\overline{M_{-1} F}}\right) \\
& =\mathcal{L}_{G_{c}} \sum_{i \in \mathbb{Z}} \mathcal{L}_{M_{c_{i}} F}^{-1} \mathcal{D}^{k} \mathcal{M}_{c_{i}}\left(\mathbb{I}\left\{c_{i}>0\right\} p_{\bar{F}}+\mathbb{I}\left\{c_{i}<0\right\} p_{\overline{M_{-1} F}}\right) .
\end{aligned}
$$

This expresses the asymptotic expansion as sums and products of matrices. As we did in Barbe and McCormick (2005), this method 
of calculation is suitable for computer implementation. It allows one to automatize asymptotic expansion for this type of weighted heavy tail convolutions.

In the next two examples, we will work out the details and show how to obtain expansions with as many terms as the integrability condition $m<\alpha$ and computer memory allow.

Distributions with asymptotic expansion of the form $\sum_{i \geqslant 0} a_{i} t^{-\alpha_{i}}$. The Hall-Weissman (1997) distributions are defined by

$$
\bar{F}(t)=a t^{-\alpha}+b t^{-\beta},
$$

with $\alpha<\beta$ and for $t$ at least some $t_{a, b}$. Obviously, they have an expansion of the form $\sum_{i \geqslant 0} a_{i} t^{\alpha_{i}}$.

The Burr distributions with positive paramters $\tau$ and $\gamma$ are defined by

$$
\bar{F}(t)=\left(1+t^{\tau} / \beta\right)^{-\gamma}, \quad t \geqslant 0
$$

When $\gamma$ is 1 , it is also called the log-logistic distribution. A tail expansion is easily derived. Indeed, $\bar{F}(t)=\beta^{\gamma} t^{-\gamma \tau}\left(1+\beta t^{-\tau}\right)^{-\gamma}$, and the expansion

$$
\bar{F}(t) \sim \beta^{\gamma} \sum_{k \geqslant 0}(-1)^{k} \beta^{k} \frac{\Gamma(\gamma+k)}{k ! \Gamma(\gamma)} t^{-\tau \gamma-k \tau}
$$

follows. This expansion is of the form $\sum_{i \geqslant 0} a_{i} t^{-\alpha_{i}}$.

The Fréchet distribution is defined by $\bar{F}(t)=1-\exp \left(-t^{-\alpha}\right)$. Its asymptotic expansion is given by

$$
\bar{F}(t) \sim \sum_{k \geqslant 1} \frac{(-1)^{k+1}}{k !} t^{-\alpha k} .
$$

Again, this expansion has the form $\sum_{i \geqslant 0} a_{i} t^{-\alpha_{i}}$.

So, it is of some interest here to consider an increasing sequence $0<\alpha_{0}<\ldots<\alpha_{p}$ of real numbers, and investigate expansions for weighted convolutions of distribution funcitons having an asymptotic expansion in the scale $t^{-\alpha_{i}}, 0 \leqslant i \leqslant p$. For a more comprehensive list of heavy tailed distributions with examples of their use in modeling, particularly for insurance risk data, we refer to Beirlant et al. (1996), Embrechts et al. (1997) and Rolski et al. (1999). 
Before proceeding, we need to note several things. There is of course no loss of generality in assuming that the coefficient of $t^{-\alpha_{0}}$ in the tail expansion of $\bar{F}$ does not vanish. Hence, we can assume that $\bar{F}(t) \asymp t^{-\alpha_{0}}$. We will apply Theorem 2.3.1 with $k=0$. This requires $m<\alpha_{0}$; and so, up to truncating the sequence, we can also assume that $\alpha_{p} \leqslant \alpha_{0}+m<2 \alpha_{0}$. The next thing to notice is that the asymptotic scale $\left(t^{-\alpha_{i}}\right)_{0 \leqslant i \leqslant p}$ may not be a $\star$-asymptotic scale. Indeed, it is closed under differentiation if and only if for any integer $0 \leqslant i \leqslant p$ and any nonnegative integer $k$ for which $\alpha_{i}+k \leqslant \alpha_{p}$, there exists a $j$ such that $\alpha_{i}+k=\alpha_{j}$. If this is not the case, we enlarge our collection of $\alpha_{j}$ 's by adding the missing ones recursively. Thus, we assume without loss of generality that $\left(t^{-\alpha_{i}}\right)_{0 \leqslant i \leqslant p}$ is a $\star$-asymptotic scale.

Now, let us consider the functions $e_{i}(t)=t^{-\alpha_{i}}, 0 \leqslant i \leqslant p$. They form the basis of a finite dimensional vector space, isomorphic to $\mathbb{R}^{p+1}$. Since $\mathrm{D} e_{i}=-\alpha_{i} e_{j}$ with $j$ defined by $\alpha_{j}=\alpha_{i}+1$, the derivative is identified to the matrix $\mathcal{D}$ defined by

$$
\mathcal{D} e_{i}= \begin{cases}-\alpha_{i} e_{j} & \text { if } \alpha_{j}=\alpha_{i}+1 \leqslant \alpha_{p} \\ 0 & \text { otherwise }\end{cases}
$$

Since $\mathcal{D}$ maps $e_{i}$ into the space spanned by $e_{i+1}, \ldots, e_{p}$, it is a nilpotent matrix.

Next, since $M_{c} e_{i}=c^{\alpha_{i}} e_{i}$, the matrix $\mathcal{M}_{c}$ is the diagonal matrix $\operatorname{diag}\left(c^{\alpha_{i}}\right)_{0 \leqslant i \leqslant p}$.

To show how this works practically, we consider the Burr distribution with $\gamma=10$ and $\tau=3 / 2$ say (other values would work just as well). Then $\alpha_{0}=\gamma \tau=15$. Then, Theorem 2.3.1 allows for a 14 terms expansion of the weighted convolution. However, we limit the expansion by chosing $m=4$ in Theorem 2.3.1, though it will be clear at the end that to do so is motivated not by any difficulty in obtaining the terms in such high order expansion, but rather by concern with the space and display of such expansion. The Maple code that we used to implement the formal calculations is given in the appendix.

So we choose $m$ to be 4 . Display (3.2.1) shows that $\bar{F}$ has an asymptotic expansion in the scale $t^{-15-3 i / 2}, i \geqslant 0$. Since we want 4 terms and $t^{-4} \bar{F}(t) \asymp t^{-19}$, we can restrict the range of $i$ 's to $15+3 i / 2 \leqslant 19$, that is $i \leqslant 2$. The expansion of $\bar{F}$ is then

$$
\bar{F}(t)=\beta^{10} t^{-15}-10 \beta^{11} t^{-33 / 2}+55 \beta^{12} t^{-18}+o\left(t^{-19}\right) .
$$


So we need to consider the functions $t^{-15}, t^{-15-3 / 2}$ and $t^{-18}$. This family is not closed under differentiation modulo terms in $o\left(t^{-19}\right)$. To close it, we need to add the derivative of $t^{-15}$, that is up to a multiplicative constant, $t^{-16}$. But then we need to add the derivative of $t^{-16}$ as well, that is to add $t^{-17}$. And similarly, we must add $t^{-19}$. Next we also need to add the derivative of $t^{-15-3 / 2}$, which is up to scale $t^{-18-1 / 2}$. This process leads to the $\star$-asymptotic scale $\left(e_{i}\right)_{0 \leqslant i \leqslant 7}$ corresponding to the following $\alpha_{i}$ 's:

$$
\alpha_{0}=15<16<16+1 / 2<17<17+1 / 2<18<18+1 / 2<19 .
$$

Since we have eight $\alpha_{i}$ 's, we need to work in the space $\mathbb{R}^{8}$.

Display (3.2.2) shows that

$$
\bar{F}=\beta^{10} e_{0}-10 \beta^{11} e_{2}+55 \beta^{12} e_{5}+o\left(e_{7}\right) .
$$

So, the vector $p_{\bar{F}}$ in $\mathbb{R}^{8}$ is simply

$$
p_{\bar{F}}=\left(\beta^{10}, 0,-10 \beta^{11}, 0,0,55 \beta^{12}, 0,0\right) .
$$

Since $\mathrm{D} e_{0}=-15 e_{1}$ and $\mathrm{D} e_{i}=-\alpha_{i} e_{i+2}$ for $i$ at least 1 , the matrix that corresponds to the differentiation is defined by

$$
\mathcal{D}=\left(\begin{array}{cccccccc}
0 & & & & & & & \\
-15 & 0 & & & 0 & & & \\
0 & 0 & 0 & & & & & \\
& -16 & 0 & 0 & & & & \\
& & -16.5 & 0 & 0 & & & \\
& & & -17 & 0 & 0 & & \\
& & & & -17.5 & 0 & 0 & \\
& & & & & -18 & 0 & 0
\end{array}\right)
$$

Moreover, since $M_{c} e_{k}=c^{\alpha_{k}} e_{k}$, the multiplication operator is represented by

$$
\mathcal{M}_{c}=\operatorname{diag}\left(c^{\alpha_{k}}\right)_{0 \leqslant k \leqslant 7} .
$$

A Laplace character $L_{K, 4}$ is identified to the matrix

$$
\mathcal{L}_{K}=\sum_{0 \leqslant j \leqslant 4} \frac{(-1)^{j}}{j !} \mu_{K, j} \mathcal{D}^{j}
$$


This shows that $\mathcal{L}_{M_{c} F}$ is the matrix

$$
\left(\begin{array}{cccccccc}
1 & & & & & & & \\
15 c \mu_{1} & 1 & & & & & \\
0 & 0 & 1 & & & & & \\
120 c^{2} \mu_{2} & 16 c \mu_{1} & 0 & 1 & & & & \\
0 & 0 & \frac{33}{2} c \mu_{1} & 0 & 1 & & & \\
680 c^{3} \mu_{3} & 136 c^{2} \mu_{2} & 0 & 17 c \mu_{1} & 0 & 1 & & \\
0 & 0 & \frac{1155}{8} c^{2} \mu_{2} & 0 & \frac{35}{2} c \mu_{1} & 0 & 1 & \\
3060 c^{4} \mu_{4} & 816 c^{3} \mu_{3} & 0 & 153 c^{2} \mu_{2} & 0 & 18 c \mu_{1} & 0 & 1
\end{array}\right)
$$

Finally, $\bar{G}_{c}$ has an expansion whose coefficients are given by

$$
\mathcal{L}_{G_{c}} \sum_{i \in \mathbb{Z}} \mathcal{L}_{M_{c_{i}} F}^{-1} \mathcal{M}_{c_{i}} p_{\bar{F}}
$$

We formally compute the vector $\mathcal{L}_{M_{c_{i}} F}^{-1} \mathcal{M}_{c_{i}} p_{\bar{F}}$. Assuming that

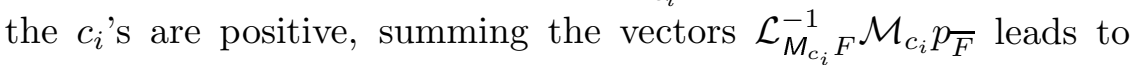
expressions involving $C_{s}=\sum_{i \in \mathbb{Z}} c_{i}^{s}$. The matrix $\mathcal{L}_{G}$ involves moments of $G_{c}$ which in turn are expressed in terms of moments of $F$ and other expressions involving the terms $C_{s}$.

We can then compute the matrix for the Laplace character associated to $G$. We then do a formal matrix multiplication. To write the final result recall the notation $C_{p ; q}$ for $C_{p+q}-C_{p} C_{q}$. We also write $\kappa_{3}$ for the third central moment of $F$, that is for $E\left(X-\mu_{F, 1}\right)^{3}$; and $\kappa_{4}$ for the fourth one, $E\left(X-\mu_{F, 1}\right)^{4}$. We then proved that $P\{\langle c, X\rangle>t\}$ has asymptotic expansion $\sum_{0 \leqslant i \leqslant 7} q_{i} e_{i}$ with

$$
\begin{aligned}
q_{0}= & \beta^{10} C_{15}, \\
q_{1}= & -15 \beta^{10} \mu_{1} C_{15 ; 1}, \\
q_{2}= & -10 \beta^{11} C_{33 / 2}, \\
q_{3}= & 120 \beta^{10} \mu_{1}^{2}\left(C_{17 ; 1}-C_{1} C_{15 ; 1}\right)-120 \beta^{10} \sigma^{2} C_{15 ; 2}, \\
q_{4}= & 165 \beta^{11} \mu_{1} C_{33 / 2 ; 1} \\
q_{5}= & -680 \beta^{10} \mu_{1}^{3}\left(C_{17: 1}-2 C_{1} C_{16 ; 1}+C_{1}^{2} C_{15 ; 1}\right) \\
& +2040 \beta^{10} \sigma^{2} \mu_{1}\left(C_{17 ; 1}-C_{2} C_{15 ; 1}-680 \beta^{10} \kappa_{3} C_{15 ; 3}+55 \beta^{12} C_{18},\right. \\
q_{6}= & \frac{5775}{4} \beta^{11}\left(-\mu_{1}^{2}\left(C_{35 / 2 ; 1}-C_{1} C_{33 / 2 ; 1}\right)+\sigma^{2} C_{33 / 2 ; 2}\right) \\
q_{7}= & 3060 \beta^{10} \mu_{1}^{4}\left(C_{18 ; 1}-3 C_{1} C_{17 ; 1}-3 C_{1}^{2} C_{16 ; 1}-C_{1}^{3} C_{15 ; 1}\right)
\end{aligned}
$$




$$
\begin{aligned}
& -18360 \beta^{10} \sigma^{2} \mu_{1}^{2}\left(C_{18 ; 1}-C_{1} C_{17 ; 1}-C_{2} C_{16 ; 1}-C_{1} C_{2} C_{15 ; 1}\right) \\
& -9180 \beta^{10} \sigma^{4}\left(2 C_{17 ; 2}-C_{15} C_{2 ; 2}\right) \\
& +12240 \beta^{10} \kappa_{3} \mu_{1}\left(C_{18 ; 1}-C_{3} C_{15 ; 1}\right) \\
& -990 \beta^{12} \mu_{1} C_{18 ; 1}-3060 \beta^{10} \kappa_{4} C_{15 ; 4} .
\end{aligned}
$$

It should be clear now that Theorem 2.3.1 is not a pure abstraction and that the algebraic nature of the Laplace character is what makes such a computation possible.

The log-gamma distribution. Recall that $X$ has a log-gamma distribution with parameter $(\lambda, \alpha)$ if its density is given by

$$
f(x)=\frac{\alpha^{\lambda}}{\Gamma(\lambda)}(\log x)^{\lambda-1} x^{-\alpha-1}, \quad x \geqslant 1 .
$$

This is equivalent to saying that $X=\exp (Z / \alpha)$ where $Z$ has a standard Gamma distribution with parameter $\lambda$. Successive integrations by parts show that

$$
P\{Z>t\} \sim \frac{e^{-t}}{\Gamma(\lambda)} \sum_{k \geqslant 0}(\lambda-1)_{k} t^{\lambda-1-k} .
$$

The distribution function $F$ of $X$ is given by $P\{Z \leqslant \alpha \log x\}$. Thus, it has expansion

$$
\bar{F}(t) \sim \frac{1}{\Gamma(\lambda) t^{\alpha}} \sum_{k \geqslant 0}(\lambda-1)_{k} \alpha^{\lambda-1-k}(\log t)^{\lambda-1-k} .
$$

This is an expansion in the scale $e_{k}(t)=t^{-\alpha}(\log t)^{\lambda-1-k}, k \geqslant 0$.

Since

$$
e_{k}^{\prime}(t)=t^{-\alpha-1}(\log t)^{\lambda-1-k}\left(-\alpha+\frac{\lambda-1-k}{\log t}\right),
$$

we see that $e_{k}^{\prime}=o\left(e_{i}\right)$ at infinity for any $i$. Consequently, any finite family $\left(e_{i}\right)_{0 \leqslant i \leqslant p}$ is closed under differentiation up to $o\left(e_{p+k}\right)$, $k \geqslant 1$. Moreover $\mathrm{D} e_{i}=o\left(e_{p}\right)$ implies that the matrix corresponding to derivative, $\mathcal{D}$, is 0 .

To determine the matrices $\mathcal{M}_{c}$, we write

$$
\begin{aligned}
e_{j}(t / c) & =c^{\alpha} t^{-\alpha}(\log t)^{\lambda-1-j}\left(1-\frac{\log c}{\log t}\right)^{\lambda-1-j} \\
& \sim \sum_{k \geqslant 0} \frac{(\lambda-1-j)_{k}}{k !}(-1)^{k} c^{\alpha}(\log c)^{k} e_{j+k}(t) .
\end{aligned}
$$


Hence, the matrix $\mathcal{M}_{c}$ is the lower triangular matrix defined by

$$
\left(\mathcal{M}_{c}\right)_{i, j}= \begin{cases}\frac{(\lambda-1-j)_{i-j}}{(i-j) !}(-1)^{i-j} c^{\alpha}(\log c)^{i-j} & \text { if } i \geqslant j \geqslant 0, \\ 0 & \text { otherwise. }\end{cases}
$$

(Note that we indexed our matrix so that the first row and column are labeled 0.) In particular $\left(e_{k}\right)_{k \geqslant 0}$ is a $\star$-asymptotic scale. Since $\mathcal{D}$ vanishes, the Laplace character of $F$ is the identity. So the formula in Theorem 2.3.1 yields for any $\alpha$ more than 1 ,

$$
G_{c}(t) \sim \sum_{i \in \mathbb{Z}} \mathcal{M}_{c_{i}} p_{\bar{F}}
$$

with

$$
p_{\bar{F}}=\frac{\alpha^{\lambda-1}}{\Gamma(\lambda)}\left(1, \frac{\lambda-1}{\alpha}, \frac{(\lambda-1)_{2}}{\alpha^{2}}, \ldots, \frac{(\lambda-1)_{p}}{\alpha^{p}}\right) .
$$

Writing $(C \log C)_{r, s}$ for $\sum_{i \in \mathbb{Z}} c_{i}^{r}\left(\log c_{i}\right)^{s}$, we obtain for instance the asymptotic expansion

$$
\bar{G}_{c} \sim \sum_{j \geqslant 0} q_{j} e_{j}
$$

with

$$
q_{j}=\frac{\alpha^{\lambda-1}}{\Gamma(\lambda)} \sum_{0 \leqslant i \leqslant j} \frac{(\lambda-1)_{j-i}}{\alpha^{j-i}} \frac{(\lambda-1-j+i)_{i}}{i !}(-1)^{i}(C \log C)_{\alpha, i} .
$$

The interesting feature of this example is that for any $m$, the identification $\mathcal{L}_{F}$ of the $m$-th Laplace character of $F$ is the identity. A consequence is that in this asymptotic scale, increasing $m$ in Theorem 2.3.1 does not yield extra terms compared to taking $m$ to be 0 . This is entirely due to the choice of the asymptotic scale. However, the expansion given in Theorem 2.3.1 provides in fact a better estimate. The reason is that the asymptotic scale in Theorem 2.3.1 is adapted to the distribution function $F$ and its derivatives. It is not a scale given a priori on which $\bar{F}$ is expanded.

This situation is very similar to the fact that the normal tail

$$
\bar{\Phi}(t)=\int_{t}^{\infty} \frac{e^{-x^{2} / 2}}{\sqrt{2 \pi}} \mathrm{d} x
$$

has a one term expansion in the scale $\bar{\Phi}^{k}, k \geqslant 1$, say, but it has an expansion given by a divergent series in the asymptotic scale $t^{-k} e^{-t^{2} / 2}, k \geqslant 0$. 
A degenerate case. For the log-gamma distribution, we saw that we can find an asymptotic scale such that Theorem 2.3.1 with $m$ equal 0 provides as many terms as we like. In the example to be developed now, we show that the reverse situtation may occur; that is, due to rather exceptional cancellations, there are distributions such that Theorem 2.3.1 provides only 1 term. This was the statement ending subsection 3.1. For simplicity we will show this only when the $c_{i}$ 's are nonnegative. It is conceptually easy to adapt the proof if this does not hold.

So, let us first choose $m$, as large as we want. Let $\alpha$ be larger than $m$, and let $\left(c_{i}\right)_{i \in \mathbb{Z}}$ be a sequence of nonnegative numbers such that $N_{\alpha, \gamma, \omega}(c)$ is finite. We consider the $\star$-asymptotic scale $e_{i}(t)=t^{-\alpha-i}$, $0 \leqslant i \leqslant m$. As we have seen in the first example of this subsection, the derivative $\mathrm{D}$ is identified with the matrix $\mathcal{D}$ whose entries are

$$
\mathcal{D}_{i, j}= \begin{cases}-\alpha-j & \text { if } i=j+1 \\ 0 & \text { otherwise }\end{cases}
$$

Moreover, for any $c$ positive,

$$
\mathcal{M}_{c}=\operatorname{diag}\left(c^{\alpha}, c^{\alpha+1}, \ldots, c^{\alpha+m}\right) .
$$

As before, the $m$-th Laplace character of $F$ is identified with the matrix

$$
\mathcal{L}_{F, m}=\sum_{0 \leqslant j \leqslant m} \frac{(-1)^{j}}{j !} \mu_{F, j} \mathcal{D}^{j} .
$$

Let $p=\left(p_{0}, \ldots, p_{m}\right)$ be the coefficients of the expansion of $\bar{F}$ in the asymptotic scale $e_{i}, 0 \leqslant i \leqslant m$. Then, the coefficients of $\bar{G}_{c}$ in this asymptotic scale are given by

$$
q=\mathcal{L}_{G_{c}, m} \sum_{i \in \mathbb{Z}} \mathcal{L}_{M_{c_{i}} F, m}^{-1} \mathcal{M}_{c_{i}} p
$$

So, it suffices to prove that we can find $F$ such that $q=e_{0}$. Note that there is no loss of generality in assuming all the first $m$ moments of $F$ fixed, because fixing these moments does not put any restriction on the vector $p$. Hence the first $m$ moments of $G_{c}$ are fixed. In other words both the Laplace characters of $F$ and $G_{c}$ can be taken fixed. We already know that $\mathcal{L}_{G_{c}, m}$ is invertible for it is a lower triangular matrix with all diagonal terms equal to 1 . Along the lines of Proposition 2.1.3 we obtain an expression for the inverse of $\mathcal{L}_{M_{c_{i}} F}$. 
Specifically, we define the nilpotent matrix $\mathcal{N}=\operatorname{Id}-\mathcal{L}_{M_{c_{i}} F, m}$ and have

$$
\mathcal{L}_{M_{c_{i}} F, m}^{-1}=(\mathrm{Id}-\mathcal{N})^{-1}=\sum_{0 \leqslant j \leqslant m} \mathcal{N}^{j}
$$

is also lower triangular, with its diagonal elements all equal to 1. Consequently, the diagonal elements of $\mathcal{L}_{\mathcal{M}_{c_{i}} F, m}^{-1} \mathcal{M}_{c_{i}}$ are those of $\mathcal{M}_{c_{i}}$. Hence the matrix $\sum_{i \in \mathbb{Z}} \mathcal{L}_{M_{c_{i} F, m}}^{-1} \mathcal{M}_{c_{i}}$ is lower triangular, with diagonal elements $\left(C_{\alpha}, C_{\alpha+1}, \ldots, C_{\alpha+m}\right)$. It is invertible. Therefore it is possible to find $p$ with positive $e_{0}$ component such that $q$ is $e_{0}$. This shows the existence of a distribution function $F$ such that the formula in Theorem 2.3.1 yields only a 1 term expansion.

This discussion is particularly relevant to the problem of ascertaining second order regular variation for infinite order moving averages. If an $m$-term expansion shows that, for example, $\bar{G}_{c} \sim \mathrm{Id}^{-\alpha}+\mathrm{Id}^{-\alpha-m}$, then $\bar{G}_{c}$ is second-order regularly varying; but this would not be revealed until an $m$-terms expansion was calculated. Thus, we see that second order regular variation for linear processes is not a second order expansion question; rather, it is a higher order expansion question. We discuss this problem in detail in the next section.

3.3. Two terms expansion and second order regular variation. Motivated by probabilist and statistical applications, consider the following problem: If $\bar{F}$ is regularly varying of index $-\alpha$ with remainder, what is an asymptotic equivalent of $\bar{G}_{c}-C_{\alpha} \bar{F}$ ?

In this section, we will first explain this problem and the terminology used, and then show that Theorem 2.3.1 sheds an interesting light on the matter.

Recall that $\bar{F}$ is regularly varying with index $-\alpha$ and remainder, if there exist functions $k(\cdot)$ and $g(\cdot)$, with $g$ tending to 0 at infinity, such that

$$
\frac{\bar{F}(\lambda t)}{\bar{F}(t)}-\lambda^{-\alpha} \sim \lambda^{-\alpha} k(\lambda) g(t)
$$

as $t$ tends to infinity — see, Bingham, Goldie and Teugels (1989, $\S 3.12$ ). Note that this relation does not change if we multiply $g$ and divide $k$ by the same constant. If this relation holds, then $g$ must be regularly varying with nonpositive index $\rho$ and, up to a possible multiplication of $g$ by a constant, necessarily

$$
k(\lambda)= \begin{cases}\left(\lambda^{\rho}-1\right) / \rho & \text { if } \rho<0 \\ \log \lambda & \text { if } \rho=0\end{cases}
$$


We then write $\bar{F} \in 2 R V(-\alpha, g)$. Unless otherwise specified, we assume for simplicity that the $c_{i}$ 's are nonnegative. The problem mentioned can be rephrased as: assuming (3.3.1), we know that $\bar{F}$ is regularly varying with index $-\alpha$, hence that

$$
\lim _{t \rightarrow \infty} \bar{G}_{c}(t) / \bar{F}(t)=C_{\alpha} .
$$

What is the exact rate of convergence in this limit? One would indeed think that adding one term to the regular variation as in (3.3.1) brings one more term in the asymptotic expansion for $\bar{G}_{c}$. The examples in subsection 3.1 show that this belief is not correct in general.

Since the problem is motivated by applications in time series where it is natural to suppose that $F$ is centered, we assume that $\mu_{F, 1}$ vanishes - the following discussion can easily be modified if this first moment does not vanish. In order to apply Theorem 2.3.1 with $k=0$ and $m=2$, we assume that $\bar{F}$ is smoothly varying of order larger than 2 and that $\alpha$ is larger than 2 . We calculate the second Laplace character $L_{G_{c} \sharp M_{c_{i}} F, 2}=\mathrm{Id}+(1 / 2) \mu_{F, 2}\left(C_{2}-c_{i}^{2}\right) \mathrm{D}^{2}$. Then Theorem 2.3.1, with $m=2$ and $k=0$, and the fact that $\bar{F}^{\prime \prime}(t) \sim \alpha(\alpha+1) t^{-2} \bar{F}(t)$ at infinity, yield

$$
\bar{G}_{c}(t)=\sum_{i \in \mathbb{Z}} \overline{M_{c_{i}} F(t)}-\frac{\alpha(\alpha+1)}{2} C_{\alpha ; 2} \mu_{F, 2} t^{-2} \bar{F}(t)+o\left(t^{-2} \bar{F}(t)\right)
$$

as $t$ tends to infinity. Consequently,

$$
\frac{\bar{G}_{c}}{\bar{F}}(t)-C_{\alpha}=\sum_{i \in \mathbb{Z}}\left(\frac{\bar{F}\left(t / c_{i}\right)}{\bar{F}(t)}-c_{i}^{\alpha}\right)-\frac{\alpha(\alpha+1)}{2} C_{\alpha ; 2} \mu_{F, 2} t^{-2}+o\left(t^{-2}\right) .
$$

The global Potter type bounds of Theorem 3.1.3 in Bingham, Goldie and Teugels (1989) and (3.3.1) imply

$$
\begin{aligned}
\frac{\bar{G}_{c}}{\bar{F}}(t)-C_{\alpha}=g(t) \sum_{i \in \mathbb{Z}} c_{i}^{\alpha} k\left(1 / c_{i}\right) & (1+o(1)) \\
& -\frac{\alpha(\alpha+1)}{2} C_{\alpha ; 2} \mu_{F, 2} t^{-2}+o\left(t^{-2}\right) .
\end{aligned}
$$

Consequently, one sees that the second order term depends on the behavior of $t^{2} g(t)$ at infinity. If $\lim _{t \rightarrow \infty} t^{2} g(t)=\infty$, then

$$
\frac{\bar{G}_{c}}{\bar{F}}(t)-C_{\alpha} \sim g(t) \sum_{i \in \mathbb{Z}} c_{i}^{\alpha} k\left(1 / c_{i}\right)
$$


while if $\lim _{t \rightarrow \infty} t^{2} g(t)=0$ then

$$
\frac{\bar{G}_{c}}{\bar{F}}(t)-C_{\alpha} \sim-\frac{\alpha(\alpha+1)}{2} C_{\alpha ; 2} \mu_{F, 2} t^{-2},
$$

with the usual convention that if the constant $C_{\alpha ; 2}$ in the right hand side vanishes, then the right hand side should be read as $o\left(t^{-2}\right)$. If $g(t) \sim a t^{-2}$, then $\rho$ is -2 and we obtain

$$
\frac{\bar{G}_{c}}{\bar{F}}(t)-C_{\alpha} \sim \frac{1}{2 t^{2}}\left(-a\left(C_{\alpha+2}-C_{\alpha}\right)-\alpha(\alpha+1) C_{\alpha ; 2} \mu_{F, 2}\right) .
$$

If the constant in the second order terms vanishes (which generically does not happen), then if we can, we need to add one more term when applying Theorem 2.3.1. But one should be careful that the rate of convergence of $t^{2} g(t)$ to its limit may cancel the extra term added. If cancellation occurs, then more terms are needed, and so on. It is therefore not clear that second order regular variation provides the right framework for studying second order expansions of $\bar{G}_{c}$. In particular, for some exceptional sequences of constants and some distributions, higher order regular variation will be needed to obtain the exact second order. We also would like to point out that smooth variation of finite order is far easier to check than second order regular variation, and that it holds for most - if not all heavy tail distributions used in practical applications.

We conclude this section with a somewhat more general result to illustrate how weights of arbitrary signs appear in the expansion. If $X$ is a random variable with distribution function $F$, we write $F_{*}$ the distribution function of $|X|$. Thus, on the nonnegative half line, $\bar{F}_{*}=\overline{M_{-1} F}+\bar{F}$. In the statistical literature dealing with regular variation for the upper and lower tails of distributions, it is customary to replace (3.3.1) by a second order regular variation assumption on $\bar{F}_{*}$, that is, with the same notation as in (3.3.1),

$$
\frac{\bar{F}_{*}(\lambda t)}{\bar{F}_{*}(t)}-\lambda^{-\alpha} \sim \lambda^{-\alpha} k(\lambda) g(t),
$$

and a tail balancing condition with remainder, that is for some nonnegative $p$ at most 1 ,

$$
\bar{F}=p \bar{F}_{*}+o\left(\bar{F}_{*} g\right)
$$


at infinity. Set $q=1-p$ and

$$
\kappa_{c}(\lambda)=\sum_{i \in \mathbb{Z}}\left(\lambda /\left|c_{i}\right|\right)^{-\alpha} k\left(\lambda /\left|c_{i}\right|\right)\left(p \mathbb{I}\left\{c_{i}>0\right\}+q \mathbb{I}\left\{c_{i}<0\right\}\right) .
$$

Define the constants

$$
C_{+, \alpha}=\sum_{\substack{i \in \mathbb{Z} \\ c_{i}>0}} c_{i}^{\alpha} \quad \text { and } C_{-, \alpha}=\sum_{\substack{i \in \mathbb{Z} \\ c_{i}<0}}\left|c_{i}\right|^{\alpha} .
$$

Furthermore, set

$$
C_{*, \alpha}=p C_{+, \alpha}+q C_{-, \alpha}
$$

and

$$
C_{*, \alpha ; 1}=p\left(C_{+, \alpha+1}-C_{1} C_{+, \alpha}\right)-q\left(C_{-, \alpha+1}+C_{1} C_{-, \alpha}\right) .
$$

Assume that $\alpha$ is larger than 1 and that $F$ satisfies the assumptions of Theorem 2.3.1with $m=1$ and $k=0$. Finally, assume also that $a=\lim _{t \rightarrow \infty} t g(t)$ exists, possibly infinite. Then,

$$
\begin{aligned}
& \frac{\bar{G}_{c}(\lambda t)}{\bar{F}_{*}(t)}-C_{*, \alpha} \lambda^{-\alpha} \\
& \sim \begin{cases}\left(a \kappa_{c}(\lambda)-\lambda^{-\alpha-1} \alpha \mu_{F, 1} C_{*, \alpha ; 1}\right) t^{-1} & \text { if } a \text { is finite, } \\
\kappa_{c}(\lambda) g(t) & \text { if } a \text { is infinite. }\end{cases}
\end{aligned}
$$

We remark that the above results extend Theorem 3.2.III in Geluk et al. (1997).

3.4. Some open questions. Formula (3.1.3) provides generically a two terms expansion when the mean $\mu_{F, 1}$ vanishes. For this expansion to be valid, we need a variance in order to define the second Laplace character. Consequently, we do not know what a two terms expansion is when the distribution pertaining to $F$ is centered and has infinite variance; that is essentially in the range $\alpha$ between 1 and 2 .

When $\alpha$ is less than 1 , the mean does not exists, and we only have the classical equivalence $\bar{G}_{c} \sim \sum_{i \in \mathbb{Z}} \overline{M_{c_{i}} F}$. Some examples in subsection 3.1 and the log-gamma distribution studied in subsection 3.2 suggest that in some instances this equivalent may still provide two terms or more. 
When $\alpha$ is less than 1 and $F$ is concentrated on the nonnegative half line with smoothly varying tail of index $\alpha$ and order more than 1, Theorems 2.5 and 2.6 of Barbe and McCormick (2005) imply a result for some finite convolutions. Specifically, define

$$
I(\alpha)=2 \int_{0}^{1 / 2}\left((1-y)^{-\alpha}-1\right) \alpha y^{-\alpha-1} \mathrm{~d} y+2^{2 \alpha}-2^{\alpha+1} .
$$

Then, if the $c_{i}$ 's are nonnegative constants,

$$
\overline{\star_{1 \leqslant i \leqslant n} M_{c_{i}} F}=\sum_{1 \leqslant i \leqslant n} \overline{M_{c_{i}} F}+\frac{I(\alpha)}{2}\left(\sum_{1 \leqslant i, j \leqslant n} c_{i}^{\alpha} c_{j}^{\alpha}-\sum_{1 \leqslant i \leqslant n} c_{i}^{2 \alpha}\right) \bar{F}^{2} .
$$

This suggests that under suitable conditions, the tail of the series $\sum_{i \in \mathbb{Z}} c_{i} X_{i}$ should behave like

$$
\sum_{i \in \mathbb{Z}} \overline{M_{c_{i}} F}+\frac{I(\alpha)}{2}\left(C_{\alpha}^{2}-C_{2 \alpha}\right) \bar{F}^{2}+o\left(\bar{F}^{2}\right) .
$$

The techniques used in Barbe and McCormick (2005) combined with those of the current paper can certainly be used to prove (3.4.1). However, even in the case of a finite number of summands, when $\alpha$ is less than 1, we do not know a good formalism to remove the restriction that the support of the $M_{c_{i}} F$ 's should be in the nonnegative half line.

We believe that the techniques and formalism developed in the proof of Theorem 2.3.1 of this paper are generally useful for extending a result from finite convolutions to infinite ones. Unfortunately, at the present time, finite convolution are still difficult to work with.

\section{Applications.}

In this section we develop some applications of Theorem 2.3.1 and the tail calculus explained in section 3.2. As the section goes, these applications leave room to more and more questions; the last subsection, on implicit renewal equation, only touches on a subject which deserves further consideration.

4.1. ARMA models. ARMA models are among the most used models in statistical analysis of time series. Yet, very few facts are known on their distributions. The purpose of this subsection is to show that in some circumstances, Theorem 2.3.1 provides some basic 
information on the marginal distribution. We refer to Brockwell and Davis (1991) for the basic probabilistic and statistical aspects of these models.

To fix notation, we define the backward shift operator $B$ on sequences as follows. A sequence $x=\left(x_{i}\right)_{i \in \mathbb{Z}}$ is mapped under $B$ to the sequence whose $i$-th element is $x_{i-1}$. As usual, $B^{0}$ is the identity, $B^{-1}$ is the inverse of $B$, and $B^{k}=B B^{k-1}$. It then makes sense to consider polynomials in $B$. Having two polynomials $\Theta$ and $\Phi$, and a sequence $\epsilon=\left(\epsilon_{i}\right)_{i \in \mathbb{Z}}$ of independent and identically distributed random variables, an ARMA process $X=\left(X_{i}\right)_{i \in \mathbb{Z}}$ is defined by the relation

$$
\Theta(B) X=\Phi(B) \epsilon
$$

For this process to be defined, we assume that $\Theta$ has all its roots outside the closed unit disk of the complex plane. We can define $\Theta(B)^{-1}$ by a series expansion. Then, under a mild integrability condition on $\epsilon$, we obtain $X=\Theta(B)^{-1} \Phi(B) \epsilon$. Having a series expansion yields a representation of $X$ as an infinite order moving average

$$
X_{i}=\sum_{j \in \mathbb{N}} c_{j} \epsilon_{i-j}
$$

In general it is unknown how to calculate the marginal distribution of the process, that is the distribution of $X_{0}$. Since the roots of $\Theta$ are outside the closed unit disk, the sequence $c_{j}$ decreases exponentially fast; see e.g. Brockwell et al. $(1991, \S 3.1)$. Therefore, this sequence has finite $N_{\alpha, \gamma, \omega}$-norm, whatever $\alpha, \gamma$ and $\omega$ are in the positive half line. Theorem 2.3.1 yields immediately an expansion for the tail of the marginal distribution of the process provided the distribution of the innovations $\epsilon_{i}$ has tail smoothly varying of sufficiently large order and index.

To discuss further, let us write $G_{c}$ the marginal distribution of the process. In order to make the expansion explicit, we need to evaluate the Laplace character $L_{G_{c} \sharp M_{c_{i}} F, m}$. This requires computing the moments of $G_{c} \downarrow M_{c_{i}} F$, or, equivalently, $E\left(X_{0}-c_{i} \epsilon_{-i}\right)^{k}$ for various integers $k$. We do not know any way to obtain a nice formula for those moments in terms of the polynomials $\Theta$ and $\Phi$. In general, one needs to rely on numerical methods.

There are however two cases for which explicit calculations may be performed, namely for $\operatorname{AR}(1)$ and $\operatorname{MA}(q)$ models, that is when either $\Theta(B)=\operatorname{Id}-a B$ and $\Phi(B)=\operatorname{Id}$, or $\Theta(B)=\operatorname{Id}$ and $\Phi$ is an 
arbitrary polynomial of degree $q$. For instance, in many examples of section 3, the expansions can be expressed with coefficients involving the quantity $C_{p}=\sum_{i \in \mathbb{Z}} c_{i}^{p}$. For an $\operatorname{AR}(1)$, with autocorrelation $a$ positive and less than 1 ,

$$
C_{p}=\sum_{i \geqslant 0} a^{i p}=\left(1-a^{p}\right)^{-1},
$$

while for a $\operatorname{MA}(q)$ with $\Phi(B)=\sum_{0 \leqslant i \leqslant q} \Phi_{i} B^{i}$,

$$
C_{p}=\sum_{0 \leqslant i \leqslant q} \Phi_{i}^{p}
$$

By applying these formulas to the expression obtained in section 3.3, we obtain two terms expansions of the marginal distribution of these processes with an assumption of second order regular variation for the distribution of the innovations $\epsilon$. This will be used in the next subsection where we develop an application to statistical inference for heavy tail data.

4.2. Tail index estimation. In heavy tail analysis, a critical parameter to estimate is the index of regular variation when the marginal distribution of the data has a regularly varying tail. In this example we are concerned with observations that follow a causal linear process which we denote $\left(Y_{i}\right)_{i \in \mathbb{N}^{*}}$. Thus, we assume that there is a sequence of real number $c_{i}$ 's and a sequence of independent and identically distributed random variables $X_{i}$ 's, so that

$$
Y_{i}=\sum_{j \geqslant 0} c_{j} X_{i-j}, \quad i \in \mathbb{N}^{*} .
$$

By the first order result on tail behavior, we have that, if the distribution function of the innovations $X_{i}$ 's has regularly varying tails with index $-\alpha$, then the same is true for the marginal distribution function of the stationary linear process. The statistical problem is to estimate and find a confidence interval for $\alpha$, based on sample data.

The usual semiparametric procedure is to use the Hill (1973) estimator, defined as follows. Let $|Y|_{i, n}$ be $i$-th largest value among $\left|Y_{1}\right|, \ldots,\left|Y_{n}\right|$, so that $|Y|_{n, n} \leqslant \cdots \leqslant|Y|_{1, n}$. Let $k_{n}$ be an integer between 1 and $n$. The Hill estimator is

$$
\alpha_{n}=k_{n}\left(\sum_{1 \leqslant i \leqslant k_{n}} \log \frac{|Y|_{i, n}}{|Y|_{k_{n}+1, n}}\right)^{-1} .
$$


For this estimate to be consistent, it is necessary to take points coming from the tail of the distribution, and of course we need enough of them. This is expressed by the conditions

$$
\lim _{n \rightarrow \infty} k_{n} / n=0 \quad \text { and } \quad \lim _{n \rightarrow \infty} k_{n}=\infty
$$

To derive a nondegenerate limiting distribution, and therefore obtain asymptotic confidence intervals and tests, further hypotheses need to be imposed. These were obtained by Resnick and Stărică (1997), and until the present paper could not be verified except maybe in some very special situations. To explain what the problem is, we need to list their conditions, and this requires further notation as well some rather technical consideration.

Let $F_{*}$ be the distribution function of $\left|X_{i}\right|$. It is assumed that $F_{*}$ is second order regularly varying; that is, with the same notation as in section $3.3, F_{*}$ belongs to $2 R V(-\alpha, g)$ for some regularly varying function $g$, i.e. (3.3.1) holds with $\bar{F}_{*}$ in place of $\bar{F}$. Next, it is assumed a tail balancing condition holds, namely that for some $p$ in the closed unit interval and $q=1-p$,

$$
\bar{F}=p \bar{F}_{*}+o\left(g \bar{F}_{*}\right)
$$

and

$$
\overline{M_{-1} F}=q \bar{F}_{*}+o\left(g \bar{F}_{*}\right)
$$

at infinity. This ensures that both $\bar{F}$ and $\overline{M_{-1} F}$ are second order regularly varying, with same index $-\alpha$ and same auxiliary rate function $g$. The next assumption made is that $F$ has a density $F^{\prime}$ which is Lipschitz in mean, that is there exists a positive $\kappa$, for which

$$
\int_{\mathbb{R}}\left|F^{\prime}(x)-F^{\prime}(x+y)\right| \mathrm{d} x \leqslant \kappa y .
$$

This condition ensures that the linear process is strong mixing.

Note that so far all the conditions are on the unknown distribution of the innovations. In statistical analysis of heavy tailed time series, one takes as one's model assumptions that the innovation distribution satisfies certain properties such as we have listed above. Model assumptions are simply assumed to hold. However, conditions on the marginal distribution $G$ may be worrisome. They may be redundant, i.e. one derivable from the assumptions on $F$, or they may even be inconsistent, i.e. the hypotheses for a theorem may 
apply only to an empty class of models. For our particular tail estimation problem, we will elucidate this issue next.

The next condition required is that the marginal distribution function $G$ of the process satisfies a Von Mises condition, that is has a density $G^{\prime}$ and

$$
\lim _{t \rightarrow \infty} \frac{t G^{\prime}(t)}{\bar{G}(t)}=\alpha .
$$

Let $G_{*}$ be the distribution function of $\left|Y_{i}\right|$. It is further assumed that $\bar{G}_{*}$ is second order regularly varying of index $-\alpha$, using the notation introduced after (3.3.1),

$$
\bar{G}_{*} \in 2 R V\left(-\alpha, g_{G_{*}}\right)
$$

for some regularly varying $g_{G_{*}}$.

Yet, an other assumption is that

$$
\lim _{n \rightarrow \infty} \sqrt{k_{n}} g_{G_{*}} \circ G_{*}^{\leftarrow}\left(1-k_{n} / n\right)=0
$$

Assumptions (4.2.4), (4.2.5) and (4.2.6) are rather problematic since they do not involve the distribution function of the innovation, and may potentially put rather stringent conditions on $F$ or $k_{n}$. We will investigate that matter after stating Resnick and Stărică's result.

The last assumption is on the sequence $k_{n}$, and presents no difficulty, since this sequence is chosen by whomever uses the estimator. It is assumed that

$$
\limsup _{n \rightarrow \infty} n^{2 / 3} / k_{n}<\infty \quad \text { or } \quad \liminf _{n \rightarrow \infty} n^{2 / 3} / k_{n}>0 .
$$

Set

$$
\lambda=\frac{1}{\alpha^{2}}\left(1+2 \frac{\sum_{j \geqslant 1} \sum_{k \geqslant 0}\left|c_{k}\right|^{\alpha} \wedge\left|c_{j+k}\right|^{\alpha}}{\sum_{k \geqslant 0}\left|c_{k}\right|^{\alpha}}\right) .
$$

Theorem 4.2.1. (Resnick, Stărică, 1997). Assume that there exists some $u$ more than 1 and some positive constant $A$ such that $\left|c_{i}\right| \leqslant A u^{-i}$ for any nonnegative $i$ and that $E\left|X_{1}\right|^{d}$ is finite for some positive d less than 1.

(i) If conditions (4.2.3), (4.2.4) and (4.2.7) hold, then

$$
\sqrt{k_{n}}\left(\alpha_{n}^{-1}-\frac{n}{k_{n}} \int_{1}^{\infty} P\left\{\left|Y_{1}\right| \geqslant x G_{*}^{\leftarrow}\left(1-k_{n} / n\right)\right\} \frac{\mathrm{d} x}{x}\right)
$$


has a centered normal limiting distribution, with variance $\lambda$.

(ii) If, furthermore, (4.2.5) and (4.2.6) hold, then $\sqrt{k_{n}}\left(\alpha_{n}^{-1}-\alpha^{-1}\right)$ has a normal limiting distribution with mean 0 and variance $\lambda$.

Let us now show that Theorem 2.3.1 yields rather simple and explicit conditions which ensure that (4.2.4), (4.2.5) and (4.2.6) hold, and therefore makes Resnick and Stărică's theorem far easier to use. Before stating the result we introduce some conditions on the moving average weights. Recall at the end of section 3.2, we noted that in general higher-order expansions are required to determine the auxiliary function for an infinite order weighted average. In order that the derived auxiliary function be determined by only the second order information for the innovation distribution, certain restrictions must be met. They are encompassed in the following conditions.

Similarly to $C_{r}$, we define the notation

$$
|C|_{r}=\sum_{i \in \mathbb{Z}}\left|c_{i}\right|^{r}
$$

Note that $|C|_{r}=|c|_{r}^{r}$. Recall that $k(\cdot)$ is the function appearing in the definition of second order regular variation for $\bar{F}_{*}$ as in (3.3.1). Furthermore, the parameter $\rho$ which appear in the function $k$ is the index of regular variation of the function $g$. We will use the conditions

$$
|C|_{\alpha}+\rho \sum_{i \in \mathbb{Z}}\left|c_{i}\right|^{\alpha} k\left(1 /\left|c_{i}\right|\right) \neq 0
$$

and, with $a$ a real number to be fixed later,

$$
\begin{aligned}
a|C|_{\alpha} & +a \rho \sum_{i \in \mathbb{Z}}\left|c_{i}\right|^{\alpha} k\left(1 /\left|c_{i}\right|\right) \\
& +\alpha \rho \mu_{F, 1}(p-q)\left(C_{1} \sum_{i \in \mathbb{Z}}\left|c_{i}\right|^{\alpha} \operatorname{sign}\left(c_{i}\right)-|C|_{\alpha+1}\right) \neq 0
\end{aligned}
$$

as well as

$$
a|C|_{\alpha}+a \rho \sum_{i \in \mathbb{Z}}\left|c_{i}\right|^{\alpha} k\left(1 /\left|c_{i}\right|\right)-\alpha(\alpha+1) \mu_{F, 2}\left(C_{2}|C|_{\alpha}-|C|_{\alpha+2}\right) \neq 0 .
$$

We comment on those conditions after the following statement.

Proposition 4.2.2. Assume that $\bar{F}$ and $\overline{M_{-1} F}$ are smoothly varying of index $-\alpha$ and order more than 1 , and that they belong to 
$2 R V(-\alpha, g)$. Assume also that $F^{\prime}$ ultimately exists and is continuous. Then,

(i) G obeys the Von Mises condition (4.2.4);

(ii) If $F^{\prime \prime}$ exists and is Lebesgue integrable, then (4.2.3) holds;

Next, let $\xi=1$ if $\mu_{F, 1}$ does not vanish, and $\xi=2$ otherwise. Assume furthermore that $\bar{F}$ and $\overline{M_{-1} F}$ are of order more than $\xi$ and that $a=\lim _{t \rightarrow \infty} t^{\xi} g(t)$ exists, possibly infinite.

(iii) The function $\bar{G}_{*}$ is second order regularly varying in any of the following three cases:

case 1. $a=+\infty$ and (4.2.8) holds;

case 2. a is finite, $\mu_{F, 1}$ does not vanish, and (4.2.9) holds;

case 3. a is finite, $\mu_{F, 1}$ vanishes and (4.2.10) holds.

(iv) Moreover,

$$
g_{G_{*}} \asymp \begin{cases}g & \text { if } a \neq 0 \\ \mathrm{Id}^{-\xi} & \text { if } a=0\end{cases}
$$

and

(v) condition (4.2.6) is equivalent to

$$
\begin{cases}\lim _{n \rightarrow \infty} \sqrt{k_{n}} g \circ F_{*}^{\leftarrow}\left(1-k_{n} / n\right)=0 & \text { if } a \neq 0 \\ \lim _{n \rightarrow \infty} \sqrt{k_{n}} F_{*}^{\leftarrow}\left(1-k_{n} / n\right)^{-\xi}=0 & \text { if } a=0 .\end{cases}
$$

Remark. When $\mu_{F, 1}$ does not vanish, the result is obtained by an application of Theorem 2.3.1 with $m=1$; otherwise it requires $m=2$. The all but intuitive conditions (4.2.8)-(4.2.10) are not there for technical reasons. If those conditions are not met as specified in the Proposition, then the last statement of the Proposition - the equivalence with (4.2.6) - does not hold. We would need to use Theorem 2.3.1 with a higher $m$ or higher order regular variation, which would lead in some cases (but not always) to a different result. Note that generically, the Proposition covers all the cases; however, there are some exceptional cases where it fails. This is the very same phenomenon as that observed in subsection 3.1, and commented further toward the end of the proof of Proposition 4.2.2. This unfortunate fact commands caution when estimating tail index in time series.

Remark. To fix the ideas, if $\bar{F}(t) \asymp t^{-\alpha}$ and $g(t) \asymp t^{-\beta}$ with $\beta$ positive and less than $\xi$. In this situation, $a$ is infinite. Then (4.2.6) 
is equivalent to $k_{n}=o\left(n^{2 \beta /(\alpha+2 \beta)}\right)$. One sees that the smaller $\beta$ is, the smaller $k_{n}$ should be. In crafting a good estimator, one needs to be mindful of such restrictions when using the Hill estimator in a time series context.

Proof. (i) We first set

$$
C_{+, \alpha}=\sum_{i \in \mathbb{Z} ; c_{i}>0} c_{i}^{\alpha} \quad \text { and } C_{-, \alpha}=\sum_{i \in \mathbb{Z} ; c_{i}<0}\left(-c_{i}\right)^{\alpha}
$$

Recall the classical first order equivalence

$$
\bar{G} \sim \sum_{i \in \mathbb{Z}} \overline{M_{c_{i}} F} \sim\left(p C_{+, \alpha}+q C_{-, \alpha}\right) \bar{F}_{*} .
$$

Applying Theorem 2.3.1 with $k=1$ and $m=0$ yields

$$
\bar{G}^{\prime} \sim \sum_{i \in \mathbb{Z}}{\overline{M_{c_{i}}}}^{\prime} \sim\left(p C_{+, \alpha}+q C_{-, \alpha}\right)(-\alpha) \mathrm{Id}^{-1} \bar{F}_{*} .
$$

We then deduce (4.2.4).

(ii) follows from the fundamental theorem of calculus as well as Fubini's theorem, writing

$$
\begin{aligned}
\int\left|F^{\prime}(x)-F^{\prime}(x+y)\right| \mathrm{d} x & \leqslant \iint\left|F^{\prime \prime}(x+u)\right| \mathrm{d} x \mathbb{I}\{0 \leqslant u \leqslant y\} \mathrm{d} u \\
& \leqslant y\left|F^{\prime \prime}\right|_{1} .
\end{aligned}
$$

To prove the other statements, we first derive a two terms expansion for $\overline{G_{*}}$. We write $G_{c}$ for $G$. Thus, $M_{-1} G=G_{-c}$.

We first assume that $\mu_{F, 1}$ does not vanish. Since $\bar{G}_{*}$ coincides with $\bar{G}+\overline{M_{-1} G}$ on the positive half line, Theorem 2.3.1 and the tail balance conditions (4.2.1), (4.2.2) imply

$$
\begin{aligned}
\bar{G}_{*}= & \sum_{i \in \mathbb{Z}} L_{G_{c} \natural M_{c_{i}} F, 1} \overline{M_{c_{i}} F}+\sum_{i \in \mathbb{Z}} L_{G_{-c} \natural M_{-c_{i}} F, 1} \overline{M_{-c_{i}} F}+o\left(\mathrm{Id}^{-1} \bar{F}_{*}\right) \\
= & \sum_{i \in \mathbb{Z}}\left(\overline{M_{c_{i}} F}+\overline{M_{-c_{i}} F}\right)-\mu_{F, 1} \sum_{i \in \mathbb{Z}}\left(C_{1}-c_{i}\right) \mathrm{D}\left(\overline{M_{c_{i}} F}-\overline{M_{-c_{i}} F}\right) \\
& \quad+o\left(\mathrm{Id}^{-1} \bar{F}_{*}\right) .
\end{aligned}
$$

We note that on the positive half line

$$
\overline{M_{c_{i}} F}+\overline{M_{-c_{i}} F}=\overline{M_{\left|c_{i}\right|} F_{*}} .
$$


Moreover, because $\bar{F}$ and $\overline{M_{-1} F}$ are smoothly varying of order more than 1 , we also have

$$
\mathrm{D} \overline{M_{c_{i}} F} \sim-\alpha \operatorname{Id}^{-1} \overline{M_{c_{i}} F} .
$$

We then obtain

$$
\begin{aligned}
\bar{G}_{*}= & \sum_{i \in \mathbb{Z}} \overline{M_{\left|c_{i}\right|} F_{*}} \\
& +\alpha \mu_{F, 1} \sum_{i \in \mathbb{Z}}\left(C_{1}-c_{i}\right)(p-q) \operatorname{sign}\left(c_{i}\right) \operatorname{Id}^{-1} \overline{M_{\left|c_{i}\right|} F_{*}}+o(g) \bar{F}_{*} .
\end{aligned}
$$

Consequently, we obtain the two terms expansion

$$
\begin{aligned}
& \frac{\bar{G}_{*}}{\bar{F}_{*}}=|C|_{\alpha}+\sum_{i \in \mathbb{Z}}\left|c_{i}\right|^{\alpha} k\left(1 /\left|c_{i}\right|\right) g \\
& +\alpha \mu_{F, 1}(p-q)\left(C_{1} \sum_{i \in \mathbb{Z}} \operatorname{sign}\left(c_{i}\right)\left|c_{i}\right|^{\alpha}-|C|_{\alpha+1}\right) \operatorname{Id}^{-1}+o\left(\operatorname{Id}^{-1} \vee g\right) .
\end{aligned}
$$

Let us now consider the case where $\mu_{F, 1}$ vanishes. Again, Theorem 2.3.1 and the tail balance conditions (4.2.1), (4.2.2) imply

$$
\begin{aligned}
\bar{G}_{*}= & \sum_{i \in \mathbb{Z}} L_{G_{c} \sharp M_{c_{i}} F, 2}\left(\overline{M_{c_{i}} F}+\overline{M_{-c_{i}} F}\right)+o\left(\mathrm{Id}^{-2} \bar{F}_{*}\right) \\
= & \sum_{i \in \mathbb{Z}} \overline{M_{c_{i}} F}+\overline{M_{-c_{i}} F}+\frac{\mu_{F, 2}}{2} \sum_{i \in \mathbb{Z}}\left(C_{2}-c_{i}^{2}\right) \mathrm{D}^{2}\left(\overline{M_{c_{i}} F}+\overline{M_{-c_{i}} F}\right) \\
& \quad+o\left(\mathrm{Id}^{-2} \bar{F}_{*}\right) .
\end{aligned}
$$

Because $\bar{F}$ and $\overline{M_{-1} F}$ are smoothly varying of order more than 2 , we also have

$$
\mathrm{D}^{2} \overline{M_{c_{i}} F} \sim \alpha(\alpha+1) \mathrm{Id}^{-2} \overline{M_{c_{i}} F} .
$$

Then, up to $o\left(\operatorname{Id}^{-2} \bar{F}_{*}\right)$, the tail $\bar{G}_{*}$ is

$$
\begin{aligned}
& \sum_{i \in \mathbb{Z}} \overline{M_{\left|c_{i}\right|} F_{*}}+\frac{\mu_{F, 2}}{2} \alpha(\alpha+1) \sum_{i \in \mathbb{Z}}\left(C_{2}-c_{i}^{2}\right) \operatorname{Id}^{-2} \overline{M_{\left|c_{i}\right|} F_{*}} \\
& =\sum_{i \in \mathbb{Z}} \overline{M_{\left|c_{i}\right|} F_{*}}+\frac{\mu_{F, 2}}{2} \alpha(\alpha+1)\left(C_{2}|C|_{\alpha}-|C|_{\alpha+2}\right) \operatorname{Id}^{-2} \bar{F}_{*} .
\end{aligned}
$$

Therefore, we have the two terms expansion

$$
\begin{aligned}
\frac{\bar{G}_{*}}{\bar{F}_{*}}=|C|_{\alpha} & +\sum_{i \in \mathbb{Z}}\left|c_{i}\right|^{\alpha} k\left(1 /\left|c_{i}\right|\right) g \\
& +\frac{\alpha(\alpha+1)}{2} \mu_{F, 2}\left(C_{2}|C|_{\alpha}-|C|_{\alpha+2}\right) \mathrm{Id}^{-2}+o\left(\operatorname{Id}^{-2} \vee g\right) .
\end{aligned}
$$


In either the case $\mu_{F, 1}$ vanishes or does not vanish, we obtained an expansion of the form

$$
\frac{\bar{G}_{*}}{\bar{F}_{*}}=U+V g+W \operatorname{Id}^{-\xi}+o\left(\operatorname{Id}^{-\xi} \vee g\right) .
$$

This implies

$$
\begin{aligned}
\frac{\bar{G}_{*}(\lambda t)}{\bar{G}_{*}(t)}=\lambda^{-\alpha}+ & \lambda^{-\alpha} g(t) k(\lambda)\left(1+\rho U^{-1} V\right) \\
& +\lambda^{-\alpha} U^{-1} W\left(\lambda^{-\xi}-1\right) t^{-\xi}+o\left(t^{-\xi} \vee g(t)\right) .
\end{aligned}
$$

We now prove the result in case 1 . Indeed, if $a=\lim _{t \rightarrow \infty} t^{\xi} g(t)$ is infinite, we have

$$
\frac{\bar{G}_{*}(\lambda t)}{\bar{G}_{*}(t)}=\lambda^{-\alpha}+\lambda^{-\alpha} g(t) k(\lambda)\left(1+\rho U^{-1} V\right)+o(g) .
$$

If $1+\rho U^{-1} V$ does not vanish, which is condition (4.2.8), this implies $\bar{G}_{*}$ is second order regularly varying with auxiliary function proportional to $g$.

The stated equivalence with (4.2.6) follows in this case from the fact that $\bar{G}_{*}^{\leftarrow}(1-u) \asymp \bar{F}_{*}^{\leftarrow}(1-u)$ as $u$ tends to 0 .

This proves statements (iii), (iv) and (v) in case 1 .

Note that as we have seen in section 3.1 , if $1+\rho U^{-1} V$ vanish, we cannot conclude anything without obtaining higher order expansions, and virtually any auxiliary function may occur, either connected with higher order regular variation of $\bar{F}_{*}$ (here we are talking of third order or even higher order in exceptional cases) or the third terms in the expansion of $\bar{G}_{*}$ (or higher order terms in exceptional cases).

Cases 2 and 3 are handled in the same way.

Example. To conclude this example we illustrate the result for a particular distribution. Consider a Student innovation density

$$
f(x)=K_{\alpha}\left(1+\frac{x^{2}}{\alpha}\right)^{-(\alpha+1) / 2}, \quad x \in \mathbb{R},
$$

where $K_{\alpha}$ is the normalizing constant and where $\alpha$ is more than 2 .

The Student distribution being symmetric, (4.2.1) and (4.2.2) are obvious. 
To check the assumptions of Resnick and Stărică's theorem we use Proposition 4.2.2.

It is plain that $\bar{F}$ and $\overline{M_{-1} F}$ are smoothly varying of order at least two, because the second order derivative of $f$ is regularly varying. Also, $f$ is continuously differentiable with integrable derivative; this establish that $F^{\prime \prime}$ exists, is continuous and integrable.

To prove that $\bar{F}$ is second order regularly varying, we derive a two terms expansion, writing

$$
\begin{aligned}
\bar{F}(t) & =K_{\alpha} \int_{t}^{\infty} x^{-\alpha-1} \alpha^{(\alpha+1) / 2}\left(1+\alpha x^{-2}\right)^{-(\alpha+1) / 2} \mathrm{~d} x \\
& =\alpha^{(\alpha+1) / 2} K_{\alpha} \int_{t}^{\infty} x^{-\alpha-1}-\frac{\alpha(\alpha+1)}{2} x^{-\alpha-3}+O\left(x^{-\alpha-5}\right) \mathrm{d} x \\
& =\alpha^{(\alpha+1) / 2} K_{\alpha}\left(\frac{1}{\alpha} t^{-\alpha}-\frac{\alpha(\alpha+1)}{2(\alpha+2)} t^{-\alpha-2}+O\left(t^{-\alpha-4}\right)\right) .
\end{aligned}
$$

Consequently,

$$
\begin{aligned}
\bar{F}(\lambda t)-\lambda^{-\alpha} \bar{F}(t) & \sim \alpha^{(\alpha+1) / 2} K_{\alpha} \frac{\alpha(\alpha+1)}{2(\alpha+2)}\left(-\lambda^{-\alpha-2}+\lambda^{-\alpha}\right) t^{-\alpha-2} \\
& \sim \lambda^{-\alpha} \frac{\alpha^{2}(\alpha+1)}{2(\alpha+2)} \bar{F}(t) t^{-2}\left(1-\lambda^{-2}\right)
\end{aligned}
$$

Therefore, $\bar{F}$ belongs to $2 R V\left(-\alpha, \mathrm{Id}^{-2}\right)$.

A first order analysis shows that $G_{*}^{\leftarrow}(1-u) \asymp u^{1 / \alpha}$. Consequently, choosing $k_{n}=n^{4 \theta /(4+\alpha)}$ with $\theta$ positive less than 1 ensures that the condition listed in Proposition 4.2.2.v is satisfied. For such choice, the second assumption in (4.2.7) holds. We conclude that the distributional assumptions in the Resnick and Stărică theorem are satisfied.

An example of a process where the theorem leads to a fully explicit result is the $\mathrm{AR}(1)$ model, $X_{n}=\sum_{j \geqslant 0} r^{j} Z_{n-j}$ with $|r|$ less than 1 . In that case

$$
\lambda=\frac{1+|r|^{\alpha}}{\alpha^{2}\left(1-|r|^{\alpha}\right)} .
$$

4.3. Randomly weighted sums. In this subsection, we consider a weighted sum $\sum_{i \in \mathbb{Z}} W_{i} X_{i}$, where the weights $W=\left(W_{i}\right)_{i \in \mathbb{Z}}$ are random, independent of the $X_{i}$ 's. We also write $\langle W, X\rangle$ this series. Clearly, under some assumptions on the weights, the uniformity of 
Theorem 2.3.1 allows one to obtain an asymptotic expansion for the tail of the weighted sum given the weights, and then decondition. This can be achieved with various integrability hypotheses on $W$, according to the arguments used in the proof. The one which we provide seems to work well for the applications which we will study. In particular, it does not add any moment requirement to the distribution of the $X_{i}$ 's. In applications, it is often assumed that the weights are nonnegative. This is not strictly necessary for deriving tail expansions, but it somewhat simplifies the statements and proofs.

We will develop some applications in the next subsections.

Before stating our main result on randomly weighted sums, recall that $|\cdot|_{p}$ is the $\ell_{p}$-norm on sequences. Hence, when the sequence $W$ is nonnegative, $|W|_{p}$ is $\left(\sum_{i \in \mathbb{Z}} W_{i}^{p}\right)^{1 / p}$. This is always defined, possibly infinite.

Theorem 4.3.1. Let $F$ be a continuous distribution function, with tail $\bar{F}$ in $S R_{-\alpha, \omega}$ and such that $\overline{M_{-1} F}=O(\bar{F})$ at infinity. Let $m$ be an integer less than $\alpha$ and $\omega$. Let $\gamma$ be a positive number less than 1 and $\omega-m$. Assume that $F^{(m)}$ is bounded. Let $X=\left(X_{i}\right)_{i \in \mathbb{Z}}$ be a sequence of independent and identically distributed random variables having distribution $F$. Consider some random weights $W=\left(W_{i}\right)_{i \in \mathbb{Z}}$, independent of $X$, and such that for any $1 \leqslant k \leqslant j \leqslant m$,

$$
E\left(|W|_{1}^{j-k}|W|_{k}^{k}\right) \mathbb{I}\left\{N_{\alpha, \gamma, \omega}(W) \geqslant t\right\}=o\left(t^{-m} \bar{F}(t)\right)
$$

as $t$ tends to infinity, and

$$
E N_{\alpha, \gamma, \omega}(W)^{m+\alpha+\epsilon}<\infty .
$$

Let $K_{W}$ be the conditional distribution function of $\langle W, X\rangle$ given $W$. Then,

$$
P\{\langle W, X\rangle \geqslant t\}=\sum_{i \in \mathbb{Z}} E L_{K_{W} \sharp M_{W_{i}} F, m} \overline{M_{W_{i}} F}(t)+o\left(t^{-m} \bar{F}(t)\right)
$$

as $t$ tends to infinity.

Remark. In the proof of Theorem 4.3.1, boundedness of $F^{(m)}$ is used only to prove that for any $0 \leqslant j \leqslant m$, the map $(t, w) \mapsto$ 
$w^{-j} \bar{F}^{(j)}(t / w)$ is bounded on $[1, \infty) \times(0, \infty)$. For the conclusion of the Theorem to hold, it is enough that there exist $t_{0}$ and $M$ such that for any $t$ more than $t_{0}$, for any integer $i$ and for any integer $j$ at most $m$,

$$
\left|W_{i}^{-j} \bar{F}^{(j)}\left(t / W_{i}\right)\right| \leqslant M \quad \text { a.s. }
$$

In particular, when the $W_{i}$ 's are less than a fixed number, this is implied by $\bar{F}$ belonging to $S R_{-\alpha, \omega}$. For example, the case of the $W_{i}$ 's being Bernoulli random variables occurs in the analysis of randomly stopped sums.

Proof. Let $R$ be $1 / N_{\alpha, \gamma, \omega}(W)$. Define the sequence $c=R W$, whose elements are $c_{i}=W_{i} / N_{\alpha, \gamma, \omega}(W)$. Since $N_{\alpha, \gamma, \omega}(\cdot)$ is homogenous of degree 1 , this new random sequence satisfies $N_{\alpha, \gamma, \omega}(c)=1$. Let $G_{c}$ be the conditional distribution function of $\langle c, X\rangle$ given $c$. Let $\epsilon$ be a positive real number. Let $t_{2}$ be as in Lemma 2.2.4 as applied to the normalized regularly varying function $\bar{F}$. Furthemore, let $t_{1}$ be at least $t_{2}$, and such that the function $\eta(\cdot)$ in Theorem 2.3.1 is at most $\epsilon$ on $\left[t_{1}, \infty\right)$. We apply Theorem 2.3 .1 conditioning on $W$. So, on the event $\left\{R t>t_{1}\right\}$,

$$
\left|\bar{G}_{c}(R t)-\sum_{i \in \mathbb{Z}} L_{G_{c} \sharp M_{c_{i}} F, m} \overline{M_{c_{i}} F}(R t)\right| \leqslant(R t)^{-m} \bar{F}(R t) \epsilon .
$$

Let $K$ be the (unconditional) distribution function of $\langle W, X\rangle$. Clearly, $K(t)$ is the expected value of $G_{c}(R t)$. Taking expectation with respect to the sequence $W$ in the previous inequality,

$$
\begin{aligned}
& \left|\bar{K}(t)-\sum_{i \in \mathbb{Z}} E L_{G_{c} \sharp M_{c_{i}} F, m} \overline{M_{c_{i}} F}(R t)\right| \\
& \leqslant \epsilon t^{-m} E R^{-m} \bar{F}(R t) \mathbb{I}\left\{R t \geqslant t_{1}\right\}+E \bar{G}_{c}(R t) \mathbb{I}\left\{R t \leqslant t_{1}\right\} \\
& \quad+\sum_{i \in \mathbb{Z}} E\left|L_{G_{c} \sharp M_{c_{i}} F, m} \overline{M_{c_{i}} F}(R t)\right| \mathbb{I}\left\{R t \leqslant t_{1}\right\} .
\end{aligned}
$$

The equality

$$
L_{G_{c} \natural M_{c_{i}} F, m} \overline{M_{c_{i}} F}=L_{M_{R}\left(K_{W} \natural M_{W_{i}} F\right), m} M_{R} \overline{M_{W_{i}} F}
$$

and Lemma 2.1.5 show that the left hand side of (4.3.3) is the absolute value of $\bar{K}(t)$ minus the asymptotic expansion given in the statement of the theorem. 
To bound the right hand side of (4.3.3), the Potter bound in Lemma 2.2.4 implies that for $R t$ and $t$ at least $t_{1}$,

$$
\bar{F}(R t) / \bar{F}(t) \leqslant R^{-\alpha}\left(R^{\epsilon} \vee R^{-\epsilon}\right) .
$$

Consequently,

$$
E R^{-m} \bar{F}(R t) \mathbb{I}\left\{R t \geqslant t_{1}\right\} \leqslant E R^{-m-\alpha}\left(R^{\epsilon} \vee R^{-\epsilon}\right) \bar{F}(t) .
$$

Next, we have the obvious inequality

$$
E G_{c}(R t) \mathbb{I}\left\{R t \leqslant t_{1}\right\} \leqslant P\left\{R t \leqslant t_{1}\right\}=P\left\{N_{\alpha, \gamma, \omega}(W) \geqslant t / t_{1}\right\} .
$$

Using our integrability assumption on the weights and Markov's inequality, this is at most $o\left(t^{-m} \bar{F}(t)\right)$.

We now bound the third term in the right hand side of (4.3.3). Let $F_{*}$ be the distribution function of $\left|X_{i}\right|$ and let $H_{c}$ be the conditional one of $\sum_{i \in \mathbb{Z}} c_{i}\left|X_{i}\right|$ given $c$. Then, $\mu_{G_{c} \sharp M_{c_{i}} F, j}$ is at most $\mu_{H_{c}, j}$. Consequently,

$$
\begin{aligned}
E\left|L_{G_{c} \sharp M_{c_{i}} F, m} \overline{M_{c_{i}} F}(R t)\right| \mathbb{I}\left\{R t \leqslant t_{1}\right\} & \\
& \leqslant \sum_{0 \leqslant j \leqslant m} E \mu_{H_{c}, j}\left|\mathrm{D}^{j} \overline{M_{c_{i}} F}(R t)\right| \mathbb{I}\left\{R t \leqslant t_{1}\right\} .
\end{aligned}
$$

We then make use of the following claim which allows us to untangle the weights and the random variables $X$. It is a special case of a lemma in Chow and Teicher $(1978, \S 10.3)$. It controls the moments of a deterministically weighted sum by that of the random variables and various $\ell_{p}$-norms of the weights. Recall the notation $C_{k}=\sum_{i \in \mathbb{Z}} c_{i}^{k}$.

Claim. Let $p_{k, j}=k-1+(j-k)(j-k+1) / 2$. For any positive integer $j$ less than $\alpha$,

$$
\mu_{H_{c}, j} \leqslant \sum_{1 \leqslant k \leqslant j} 2^{p_{k, j}} \mu_{F_{*}, k} \mu_{F_{*}, 1}^{j-k} C_{1}^{j-k} C_{k}
$$

Proof. The proof is that of Chow and Teicher $(1978, \S 10.3)$ but with the constant made explicit. For any nonnegative sequence $\left(a_{i}\right)_{i \in \mathbb{Z}}$,

$$
\begin{aligned}
\left(\sum_{i \in \mathbb{Z}} a_{i}\right)^{j} & =\sum_{i \in \mathbb{Z}} a_{i}\left(a_{i}+\sum_{k \in \mathbb{Z} \backslash\{i\}} a_{k}\right)^{j-1} \\
& \leqslant 2^{j-1}\left(\sum_{i \in \mathbb{Z}} a_{i}^{j}+\sum_{i \in \mathbb{Z}} a_{i}\left(\sum_{k \in \mathbb{Z} \backslash\{i\}} a_{k}\right)^{j-1}\right) .
\end{aligned}
$$


Substituting $c_{i}\left|X_{i}\right|$ for $a_{i}$ in this inequality and taking expectation on both sides with respect to $X$,

$$
\begin{aligned}
\mu_{H_{c}, j} & \leqslant 2^{j-1}\left(\mu_{F_{*}, j} C_{j}+\mu_{F_{*}, 1} \sum_{i \in \mathbb{Z}} c_{i} E\left(\sum_{k \in \mathbb{Z} \backslash\{i\}} c_{k} X_{k}\right)^{j-1}\right) \\
& \leqslant 2^{j-1}\left(\mu_{F_{*}, j} C_{j}+\mu_{F_{*}, 1} \sum_{i \in \mathbb{Z}} c_{i} \mu_{H_{c}, j-1}\right) \\
& =2^{j-1}\left(\mu_{F_{*}, j} C_{j}+\mu_{F_{*}, 1} C_{1} \mu_{H_{c}, j-1}\right) .
\end{aligned}
$$

The claim follows by induction.

Continuing the proof of Theorem 4.3.1, that is, to bound (4.3.4) from above, we have

$$
\mathrm{D}^{j} \overline{M_{c_{i}} F}(R t)=R^{-j} W_{i}^{-j} \bar{F}^{(j)}\left(t / W_{i}\right) .
$$

Since $\bar{F}^{(j)}$ is regularly varying with index $-\alpha-j$ and bounded, since we also can assume that $t$ is at least 1 say, $\bar{F}^{(j)}(t / w)$ is at most a constant times $(w \wedge 1)^{j+\alpha-\epsilon}$. In particular, the map $(t, w) \mapsto w^{-j} \bar{F}^{(j)}(t / w)$ is bounded on $[1, \infty) \times(0, \infty)$. This fact, combined with the claim, show that (4.3.4) is at most

$$
O(1) \sum_{0 \leqslant j \leqslant m} \sum_{1 \leqslant k \leqslant j} E C_{1}^{j-k} C_{k} R^{-j} \mathbb{I}\left\{R t \leqslant t_{1}\right\}
$$

that is

$$
O(1) \sum_{0 \leqslant j \leqslant m} \sum_{1 \leqslant k \leqslant j} E\left(\sum_{n \in \mathbb{Z}} W_{n}\right)^{j-k} \sum_{n \in \mathbb{Z}} W_{n}^{k} \mathbb{I}\left\{R t \leqslant t_{1}\right\} .
$$

But one of our integrability assumptions implies that this last expression is $o\left(t^{-m} \bar{F}(t)\right)$, which concludes the proof.

4.4. Randomly stopped sums. Theorem 4.3.1 has many applications in applied probability. In this subsection, we obtain a tail expansion for randomly stopped sums. Randomly stopped sums are a basic model in insurance mathematics, e.g. in modelling total claim size. A discussion of asymptotic behavior of random sums may be found in Embrechts et al. (1997). Some practical methods for obtaining tail area approximations for compound distributions may be found in Beirlant et al. (1996). Willmot and Lin (2000) is a good source of information for compound distributions and may be 
consulted for additional references; see also Willekens (1989). We further mention Omey and Willekens $(1986,1987)$ who obtained second-order results. We also mention Geluk $(1992,1996)$ who provides second order results for subordinated probability distributions in the heavy tail case.

Let $N$ be a nonnegative random variable, independent of the $X_{i}$ 's. Define the sum $S_{n}=X_{1}+\cdots+X_{n}$, with $S_{0}=0$. Consequently, we agree that $F^{\star 0}$ is the point mass at 0 . We write $K$ for the distribution function of $S_{N}$.

Theorem 4.4.1. Assume that $F$ is a distribution function on the nonnegative half line, such that $\bar{F}$ belongs to $S R_{-\alpha, \omega}$. Let $m$ be an integer less than $\alpha$ and $\omega$. Let $\gamma$ be a positive number less than $\omega-m-k$ and 1 . If $N$ has a moment of order more than

$$
\frac{\alpha+m}{\gamma}\left(\frac{\alpha+\omega}{\alpha} \vee 2\right)+m
$$

then,

$$
\bar{K}=E N L_{F^{\star}(N-1), m} \bar{F}+o\left(\operatorname{Id}^{-m} \bar{F}\right)
$$

at infinity.

Proof. Let $W$ be the random sequence defined by $W_{i}=\mathbb{I}\{0<i \leqslant$ $N\}$. Then $S_{N}=\langle W, X\rangle$. To apply Theorem 4.3 .1 with the remark following it, we need to check its assumptions, which is to check the integrability condition on the weights. Since

$$
\frac{1}{\gamma}\left(\frac{\alpha}{\alpha+\omega} \wedge \frac{1}{2}\right)^{-1}=\frac{1}{\gamma}\left(\frac{\alpha+\omega}{\alpha} \vee 2\right),
$$

we have

$$
N_{\alpha, \gamma, \omega}(W)=N^{\frac{1}{\gamma}\left(\frac{\alpha+\omega}{\alpha} \vee 2\right)} \vee 2^{\frac{\alpha}{\alpha+\omega}} .
$$

Moreover, $|W|_{1}=N$ and $|W|_{k}^{k}=N^{k}$. So the integrability conditions are implied by

$$
E N^{\frac{m+\alpha+\epsilon}{\gamma}\left(\frac{\alpha+\omega}{\alpha} \vee 2\right)}<\infty
$$

and

$$
E N^{m} \mathbb{I}\left\{N>t^{\gamma\left(\frac{\alpha}{\alpha+\omega} \wedge \frac{1}{2}\right)}\right\}=o\left(t^{-m} \bar{F}(t)\right) .
$$

The first expectation is finite by assumption. For the second one, we apply the standard trick to prove Markov's inequality. For any $p$, the expectation is at most

$$
E N^{m+p} t^{-p \gamma\left(\frac{\alpha}{\alpha+\omega} \wedge \frac{1}{2}\right)} .
$$


Take $p$ such that the exponent of $t$ is less than $-\alpha-m$ but such that $E N^{m+p}$ is finite. Use Proposition 1.3.6 in Bingham, Goldie and Teugels (1989) to conclude that the assumptions of Theorem 4.3.1 hold. To conclude, note that for $i$ positive and less than $N$, the equalities $M_{W_{i}} F=F$ and $K \downarrow M_{W_{i}} F=F^{\star(N-1)}$ hold.

As in Barbe and McCormick (200?), using the Laplace transforms of $X_{1}$ and $N$ allows one to derive a rather neat expression. Indeed, setting

$$
\Lambda_{X}(t)=E e^{-t X} \quad \text { and } \quad \Lambda_{N}(t)=E e^{-t N},
$$

equality (2.2.1) in Barbe and McCormick (200?) yields

$$
E N L_{F^{\star}(N-1), m}=-\left.\sum_{0 \leqslant j \leqslant m} \frac{1}{j !} \frac{\mathrm{d}^{j}}{\mathrm{~d} u^{j}} \frac{\Lambda_{N}^{\prime}\left(-\log \Lambda_{X}(u)\right)}{\Lambda_{X}(u)}\right|_{u=0} \mathrm{D}^{j} .
$$

Then, the technique explained in Barbe and McCormick (200?) allows for efficient computation using computer algebra packages.

Theorem 4.4.1 has an interesting special case.

Corollary 4.4.2. Let $F$ be a distribution function satisfying the assumptions of Theorem 4.4.1. If $N$ has a Poisson distribution with parameter $a$, then

$$
\bar{K}=a L_{K, m} \bar{F}+o\left(\operatorname{Id}^{-m} \bar{F}\right)
$$

Proof. The equality

$$
\sum_{k>0} k S_{k-1}^{j} \frac{a^{k}}{k !} e^{-a}=a \sum_{k \geqslant 1} S_{k-1}^{j} \frac{a^{k-1}}{(k-1) !} e^{-a}
$$

shows that

$$
E N L_{F^{\star(N-1)}, m}=a E L_{F^{\star N}, m}=a L_{K, m} .
$$

The result follows from Theorem 4.4.1.

4.5. Queueing theory. We consider a queue of $M / G / 1$ type. This means that customers arrive with interarrival time exponentially distributed with mean $\mu$; the service has general distribution function 
$B$ with finite mean $\beta$. We assume in this application that the system is stable, that is $\beta / \mu$ is less than 1 . Define

$$
H(t)=\beta^{-1} \int_{0}^{t} \bar{B}(s) \mathrm{d} s \quad t \geqslant 0 .
$$

Set $a=\beta / \mu$. The Pollaczek-Hincin formula shows that the waiting time has the compound geometric distribution function

$$
W=(1-a) \sum_{n \geqslant 0} a^{n} H^{\star n}
$$

We refer to Bingham, Goldie and Teugels (1987, p.387) or Cohen (1972) for this derivation. If $\bar{B}$ is regularly varying at infinity, Cohen (1972) shows that so are $\bar{H}$ and $\bar{W}$. When $\bar{B}$ statisfies the assumption of Theorem 2.3.1, higher order expansions can be obtained. We mention Abate et al. (1994), Abate et al. (1995), Abate and Whitt (1997) for recent related work. See also Willekens and Teugels (1992).

Proposition 4.5.1. Assume that $B$ satisfies the assumptions of Theorem 4.4.1. Let $\Lambda_{H}$ be the Laplace transform of $H$. Then

$$
\begin{aligned}
\bar{W}=\left.a(1-a) \sum_{0 \leqslant j \leqslant m} \frac{1}{j !} \frac{\mathrm{d}^{j}}{\mathrm{~d} u^{j}}\left(1-a \Lambda_{H}(u)\right)^{-2}\right|_{u=0} \bar{H}^{j} \\
+o\left(\operatorname{Id}^{-m} \bar{H}\right)
\end{aligned}
$$

at infinity.

Proof. Let $S_{n}$ be a sum of $n$ independent and identically distributed random variables having distribution function $H$. We set $S_{0}=0$. We see that $W$ is the distribution of $S_{N}$ where $N$ has a geometric distribution with parameter $a$. The Laplace transform of $N$ is

$$
\Lambda_{N}(u)=(1-a) /\left(1-a e^{-u}\right) .
$$

Therefore, for a random variable $X$ having distribution $H$,

$$
\frac{\Lambda_{N}^{\prime}\left(-\log \Lambda_{X}(u)\right)}{\Lambda_{X}(u)}=\frac{-a(1-a)}{\left(1-a \Lambda_{X}(u)\right)^{2}} .
$$


Applying formula (4.4.1), we obtain the result.

4.6. Branching processes Consider an age dependent branching process. Basically, this refers to a Galton-Watson branching process in which the particles have a random life time governed by a distribution $F$. The process starts at time 0 with one particle; at the end of its life, it generates an average of $\rho$ particles, which themselves at death will each generate an average of $\rho$ particle, and so on. We refer to Athreya and Ney (1972, chapter 4) for a complete description of the process. It is intuitively clear that if $\rho$ is less than 1 , the socalled subcritical case, then the process will become extinct.

Let $\nu(t)$ be the expected number of particles alive at time $t$. In the subcritical case, let also $N$ be a geometric random variable with parameter $\rho$, that is $N$ is a nonnegative integer $k$ with probability $(1-\rho) \rho^{k}$.

Proposition 4.6.1. Assume that $F$ is a continuous distribution function on the positive half line, whose tail $\bar{F}$ belongs to $S R_{-\alpha, \omega}$. Let $m$ be an integer less than $\alpha$ and $\omega$. Then

$$
\nu=\rho^{-1} E N L_{F^{\star(N-1)}, m} \mathbb{I}\{N \geqslant 1\} \bar{F}+o\left(\operatorname{Id}^{-m} \bar{F}\right)
$$

at infinity.

Proof. As before, let us agree that $F^{\star 0}$ is the distribution function of the point mass at 0. Equation (4) in section IV.5 of Athreya and Ney (1972) shows that

$$
\nu=\sum_{k \geqslant 0} \rho^{k}\left(\overline{F^{\star(k+1)}}-\overline{F^{\star k}}\right) .
$$

Applying Theorem 4.4.1, we see that

$$
\sum_{k \geqslant 0} \rho^{k} \overline{F^{\star(k+1)}}=\rho^{-1}(1-\rho)^{-1} E\left(\overline{F^{\star N}} \mathbb{I}\{N \geqslant 1\}\right)
$$

has asymptotic expansion

$$
\rho^{-1}(1-\rho)^{-1} E N L_{F^{\star}(N-1), m} \mathbb{I}\{N \geqslant 1\} \bar{F}
$$

while

$$
\sum_{k \geqslant 0} \rho^{k} \overline{F^{\star k}}
$$


has asymptotic expansion

$$
(1-\rho)^{-1} E N L_{F^{\star}(N-1), m} \mathbb{I}\{N \geqslant 1\} \bar{F} .
$$

The result follows.

Let us now present an explicit expansion with $m=2$. We write $\sigma_{F}^{2}$ the variance pertaining to the distribution $F$. Direct calculation yields

$$
\begin{aligned}
\rho^{-1} E N L_{F \star(N-1), 2}= & \frac{1}{1-\rho} \mathrm{Id}-\frac{2 \rho \mu_{F, 1}}{(1-\rho)^{2}} \mathrm{D} \\
& +\frac{\rho}{(1-\rho)^{3}}\left((1-\rho) \sigma_{F}^{2}+(1+2 \rho) \mu_{F, 1}^{2}\right) \mathrm{D}^{2},
\end{aligned}
$$

so that

$$
\begin{aligned}
\nu=\frac{1}{1-\rho} \bar{F} & +\frac{2 \rho \mu_{F}}{(1-\rho)^{2}} F^{\prime} \\
& -\frac{\rho}{(1-\rho)^{3}}\left((1-\rho) \sigma_{F}^{2}+(1+2 \rho) \mu_{F, 1}^{2}\right) F^{\prime \prime}+o\left(\operatorname{Id}^{-2} F\right) .
\end{aligned}
$$

The first order term yields the result in Chover et al. (1973, p.296) and the first two terms yield the second-order result result in Grubel (1987). We refer to Pakes (1975) for related work in a subexponential setting.

Note that if the Laplace transform of $F$ is known, one could use Proposition 4.5.1 to obtain an alternative form of the asymptotic expansion.

4.7. Infinitely divisible distributions. The infinite divisible distributions are those which for any integer $n$ can be written as $n$-th convolution power of another distribution. They are also the limiting distributions of sums of $n$ independent and identically distribution random variables when the distribution is allowed to change with $n$. We refer to Feller (1971) for an introduction to the topic. These distributions are characterized through their characteristic functions, which are of the form

$$
\zeta \in \mathbb{R} \mapsto \exp \left(i \tau \zeta-\frac{\sigma^{2}}{2} \zeta^{2}+\int\left(e^{i \zeta x}-1-i \frac{\zeta x}{1+x^{2}}\right) \mathrm{d} \nu(x)\right),
$$

where $\sigma^{2}$ is nonnegative, $\tau$ is a real number, $\nu$, the so-called Lévy measure, has no mass at the origin and satifies $\int x^{2} /\left(1+x^{2}\right) \mathrm{d} \nu(x)<$ 
$\infty$. Note that throughout this subsection, $i$ denotes the imaginary unit; this is the only part in this paper where complex numbers are used.

For what follows, it is convenient to set

$$
\bar{\nu}(t)=\nu(t, \infty)
$$

Let $G_{\nu}$ be an infinitely divisible distribution function with Lévy measure $\nu$. If $\nu$ has a regularly varying tail, then $\bar{G} \sim \bar{\nu}$; see e.g. Feller (1971); see also Pakes (2004) for a related first-order result and Embrechts et al. (1979) for work in the subexponential case. Under a slightly stronger assumption, Grübel (1987) proved the two terms expansion

$$
\bar{G}_{\nu} \sim \bar{\nu}-\mu_{G, 1} \mathrm{D} \bar{\nu}
$$

at infinity. With our notation this means $\bar{G}_{\nu} \sim L_{G_{\nu}, 1} \bar{\nu}$.

The following result is then quite natural.

Proposition 4.7.1. Let $\nu$ be a measure such that $\bar{\nu}$ is smoothly varying of index $-\alpha$ and order $\omega$ and $\nu(-\infty,-t)=O(\bar{\nu}(t))$ as $t$ tends to infinity. For any integer $m$ less than $\alpha$ and $\omega$,

$$
G_{\nu}=L_{G_{\nu}, m} \bar{\nu}+o\left(\operatorname{Id}^{-m} \bar{\nu}\right) .
$$

In this expansion, the Laplace character $L_{G_{\nu}, m}$ involves the moments of $G_{\nu}$ of order at most $m$. These are finite when $\bar{\nu}$ is regularly varying of index less than $-m$ and the right tail of $\nu$ is dominant, that is under the assumptions of the Proposition. In practice, those moments can be computed by differentiating the characteristic function of $G_{\nu}$. Again, computer algebra packages are wonderful for doing this type of work.

Proof. The basic idea underlying the proof is a classical representation of infinitely divisible distributions as convolutions of two distributions, the first one having light tail, the second one being that of a randomly stopped sum with heavy tail. This makes it possible to use Theorem 4.4.1.

Let $A$ be a positive constant, and let $a$ be the $\nu$-measure of $[-A, A]^{c}$. Let $\nu_{1}$ be the measure $\nu\left(\cdot \cap[-A, A]^{c}\right)$, and let $F$ be 
the distribution function pertaining to the probability measure $\nu_{1} / a$. Define

$$
\tau_{1}=\tau-\int_{[-A, A]^{c}} \frac{x}{1+x^{2}} \mathrm{~d} \nu(x) .
$$

The function

$$
\widehat{H}(\zeta)=\exp \left(i \zeta \tau_{1}-\frac{\sigma^{2}}{2} \zeta^{2}+\int_{-A}^{A} e^{i \zeta x}-1-\frac{i \zeta x}{1+x^{2}} \mathrm{~d} \nu(x)\right)
$$

is the characteristic function of an infinitely divisible distribution function $H$. Write $\widehat{F}$ for the characteristic function of $F$ and $\widehat{G_{\nu}}$ for that of $G_{\nu}$. One has

$$
\widehat{G_{\nu}}=\widehat{H} \exp (a(\widehat{F}-1)) .
$$

Let $X=\left(X_{i}\right)_{i \geqslant 1}$ be a sequence of independent and identically distributed random variables, all having distribution $F$. They induce the partial sums $S_{n}=X_{1}+\cdots+X_{n}$, with the usual convention $S_{0}=0$. Let $N$ be a Poisson random variable, with mean $a$. Let $K$ be the distribution function of the randomly stopped sum $S_{N}$. One can check that the characteristic function of $K$ is

$$
\widehat{K}=\exp (a(\widehat{F}-1)),
$$

so that $\widehat{G_{\nu}}=\widehat{H} \widehat{K}$. Consequently, $G_{\nu}=H \star K$.

It follows from Corollary 4.4.2 that $\bar{K} \sim a L_{K, m} \bar{F}$.

The remainder of the proof is the only place in section 4 where we rely on results which we will establish in the proof of Theorem 2.3.1. We write

$$
\bar{G}(t)=\int_{-\infty}^{t / 2} \bar{K}(t-x) \mathrm{d} H(x)+\int_{-\infty}^{t / 2} \bar{H}(t-x) \mathrm{d} K(x)+\bar{H} \bar{K}(t / 2) .
$$

As observed by Grübel (1987, proof of Theorem 7), the function $\bar{H}$ decays exponentially fast to 0 at infinity. So

$$
\bar{G}(t)=\int_{-\infty}^{t / 2} \bar{K}(t-x) \mathrm{d} H(x)+o\left(t^{-m} \bar{F}(t)\right) .
$$

If follows from Theorem 5.4.1, Lemma 6.2.1 and the asymptotic expansion of $\bar{K}$ that

$$
\begin{aligned}
\bar{G}(t) & =\sum_{0 \leqslant j \leqslant m} \frac{(-1)^{j}}{j !} a \mu_{K, j} \int_{-\infty}^{t / 2} \mathrm{D}^{j} \bar{F}(t-x) \mathrm{d} H(x)+o\left(t^{-m} \bar{F}(t)\right) \\
& =\sum_{0 \leqslant j \leqslant m} \frac{(-1)^{j}}{j !} a \mu_{K, j} L_{H, m-j} \mathrm{D}^{j} \bar{F}(t)+o\left(t^{-m} \bar{F}(t)\right) .
\end{aligned}
$$


Applying Lemma 2.1.4, we conclude that

$$
\bar{G}=a L_{K \star H, m} \bar{F}+o\left(\operatorname{Id}^{-m} \bar{F}\right) .
$$

Since $K \star H$ is $G_{\nu}$ and $a \bar{F}$ ultimately coincide with $\bar{\nu}$, this concludes the proof.

\subsection{Implicit transient renewal equation and iterative sys-}

tems. A renewal equation is an integral equation of the form

$$
F-a F \star H=K,
$$

where $H$ and $K$ are given distribution functions, $a$ is a real number and $F$ is the unknown. Such equation is transient if the absolute value of $a$ is less than 1 . These equations arise in applied probability and we refer to Feller (1979) for an introduction to renewal theory and Bingham, Goldie and Teugels $(1989, \S 8.6)$ for basic results and references on the tail behaviour of the solutions. Following Goldie (1991), implicit renewal equations are equations of the same form, except that $K$ is an integral transform of $F$. Again, these equations appear in applied probability and statistics, in connection with the stationary solution of iterative systems.

The purpose of this subsection is to show that in some cases, one can derive an asymptotic expansion of the solution by solving linear equations. This method is radically different from that of Kesten (1973) or Goldie (1991); it works under a different set of assumptions, closer to the one used by Grey (1994). The appealing feature of this approach is its simplicity of implementation. The idea is very simple: expand the known function on an asymptotic scale; on this scale, the implicit renewal equation becomes a finite dimensional linear system, which can be solved by elementary linear algebraic techniques. Consequently, we obtain an analogue of the technique to solve differential equations using formal series expansions and identifying the coefficients. Of course, this technique will only work for a limited type of renewal equation.

To explain the principle, we first look at a simple renewal equation.

Standard renewal equation. Assume that $H$ and $K$ have moments of order $m$. Then the renewal equation gives $m$ equations determining the moments of $F$, that is

$$
\mu_{F, j}-a \sum_{0 \leqslant i \leqslant j}\left(\begin{array}{l}
j \\
i
\end{array}\right) \mu_{F, i} \mu_{H, j-i}=\mu_{K, j}, \quad 1 \leqslant j \leqslant m .
$$


This can be rephrased in a nicer form with Proposition 2.1.1, which combined with the renewal equation imply

$$
L_{F, m}-a L_{F, m} \circ L_{H, m}=L_{K, m}
$$

When the absolute value of $a$ is less than 1 , the operator $\operatorname{Id}-a L_{H, m}$ is not in the ideal generated by $D$. Therefore, it is invertible in $\left(\mathbb{R}_{m}[\mathrm{D}], \circ\right)$. This implies that for $|a|$ less than 1 ,

$$
L_{F, m}=\left(\mathrm{Id}-a L_{H, m}\right)^{-1} \circ L_{K, m} .
$$

So, we can calculate the Laplace character and the moments of $F$. This calculation is done by manipulating finite dimensional matices.

Iterating the renewal equation yields

$$
F=\sum_{i \geqslant 0} a^{i} K \star H^{\star i}
$$

Hence, $F$ converges to $1 /(1-a)$ at infinity and its tail is given by

$$
(1-a)^{-1}-F=\sum_{i \geqslant 0} a^{i} \overline{K \star H^{\star i}} .
$$

Define the distribution function $G$ by $\bar{G}=1-(1-a) F$. It solves the equation

$$
\bar{G}-a \overline{G \star H}=(1-a) \bar{K} .
$$

Similarly to what we did in subsection 3.2, assume that $K$ and $H$ have an asymptotic expansion on a $\star$-asymptotic scale $e$. It is then conceivable that $\bar{G}$ has an expansion in that scale. Then (4.8.3) and Theorem 2.3.1 yield, with notation analogous to that of section 3 ,

$$
p_{\bar{G}}-a\left(\mathcal{L}_{G} p_{\bar{H}}+\mathcal{L}_{H} p_{\bar{G}}\right)=(1-a) p_{\bar{K}} .
$$

That is, $p_{\bar{G}}$ is the solution of a linear system of equations, and can be made explicit by the formula

$$
p_{\bar{G}}=\left(\operatorname{Id}-a \mathcal{L}_{H}\right)^{-1}\left((1-a) p_{\bar{K}}+a \mathcal{L}_{G} p_{\bar{H}}\right) .
$$

In this formula, $\mathcal{L}_{G}$ involves the moments of $G$ and those are $1-a$ times the moments of $F$; these can be calculated by solving the linear system (4.8.1). Having an expansion for $\bar{G}$ it is then trivial to derive one for $F$. 
The only thing missing to make this rigorous is to prove that $G$ has an asymptotic expansion in the same asymptotic scale $e$ as $H$ and $K$. This is trivial because, when $a$ is positive, representation (4.8.2) shows that $G$ is the distribution function of the sum of a random variable with distribution $K$ and a randomly stopped sum having a number of summands distributed according to a geometric distribution with parameter $a$; and then Theorem 4.4.1 applies. When $a$ is negative, one uses the same argument but split the sum in (4.8.2) into one where the index $i$ is odd and one where the index is even.

An implicit renewal equation. Following Goldie (1991), Grey (1994), Grincevičius (1975) and Kesten (1973), consider the distributional equation involving random variables, $R \stackrel{\mathrm{d}}{=} Q+M R$ where $(M, Q)$ and $R$ are independent and all random variables are nonnegative. We need one important extra assumption, namely that $M$ and $Q$ are independent. It would be desirable not to make this assumption, but this would require some interesting generalization of Theorem 2.3.1. To write the implicit renewal equation equivalent to this distributional identity, let $\stackrel{\mathrm{M}}{*}$ denote the Mellin-Stieltjes convolution. That is, if $F$ and $H$ are two distribution functions,

$$
H \stackrel{\mathrm{M}}{*} F(t)=\int F(t / x) \mathrm{d} H(x) .
$$

The connection with iterative systems is that if $\left(Q_{i}, M_{i}\right), i \geqslant 1$, are independent, all distributed as $(Q, M)$, then, under suitable conditions, the sequence $R_{0}=0, R_{n}=Q_{n}+M_{n} R_{n-1}$ has a distribution converging to that of $R$, that is to $F$ - see for instance the survey article by Diaconis and Freedman (1999). We mention that first-order asymptotics for random coefficient autoregressive models is consider in Resnick and Willekens (1991).

Let $H$ and $K$ be the distribution functions of $M$ and $Q$ respectiveley. The distributional equation is equivalent to

$$
F=K \star(H \stackrel{\mathrm{M}}{*} F) .
$$

Let us assume that $K$ has a tail expansion in a $\star$-asymptotic scale $e$ as in section 3.2. Equation (4.8.5) yields two equations: one on the Laplace characters, which allows us to identify the moments of $F$, one on the tail vectors $p_{\bar{F}}, p_{\bar{K}}$, which allows one to find the tail expansion of the solution. 
For any integer $k$ for which the $k$-th moments of $K$ and $H$ exist, the moment equations are

$$
\begin{aligned}
\mu_{F, k} & =\sum_{0 \leqslant j \leqslant k}\left(\begin{array}{l}
k \\
j
\end{array}\right) \mu_{K, j} \mu_{H^{\mathrm{M}} F, k-j} \\
& =\sum_{0 \leqslant j \leqslant k}\left(\begin{array}{l}
k \\
j
\end{array}\right) \mu_{K, j} \mu_{H, k-j} \mu_{F, k-j} .
\end{aligned}
$$

These equations allow one to find the moments $\mu_{F, k}$ by induction on $k$.

If $\bar{F}$ has an expansion in the scale $e$, then (4.8.5) and Theorem 2.3.1 suggest that

$$
p_{\bar{F}}=\mathcal{L}_{H_{*}^{M} F} p_{\bar{K}}+\mathcal{L}_{K} p_{H_{*}^{M} F}
$$

Since the variables are nonnegative,

$$
\overline{H \stackrel{\mathrm{M}}{*} F}(t)=\int \bar{F}(t / x) \mathrm{d} H(x)=\int \overline{M_{x} F}(t) \mathrm{d} H(x) .
$$

Consequently,

$$
p_{\overline{H_{*}^{M} F}}=\int \mathcal{M}_{x} \mathrm{~d} H(x) p_{\bar{F}} .
$$

Thus, we obtain the tail expansion of $F$ in the scale $e$, whose coefficients are given by

$$
p_{\bar{F}}=\left(\operatorname{Id}-\mathcal{L}_{K} \int \mathcal{M}_{x} \mathrm{~d} H(x)\right)^{-1} \mathcal{L}_{H_{*}^{M} F} p_{\bar{K}}
$$

Again, for this to be justified, we only need to prove that $\bar{F}$ has an expansion in the scale $e$. Our next result gives a sufficient condition, and a complete example of application follows its proof.

Proposition 4.8.1. Assume that $K$ satistifies the assumptions of Theorem 4.3.1. If $E M_{1}^{2(\alpha+m+1)}$ is less than 1, then formula (4.8.6) holds.

Note that by Jensen's inequality, the integrability assumption in Proposition 4.8.1 implies that $E \log M_{1}$ is negative, and therefore, that the implicit renewal equation has a well defined solution.

Proof. Let $Q_{i}$ (respectively $\left.M_{i}\right), i \geqslant 1$, be a sequence of independent random variables all having the distribution $K$ (respectively $H$ ). 
Let $W_{0}=1$ and for $k$ at least 1 , let $W_{k}=M_{1} \ldots M_{k}$. Under the assumption of the Proposition, $R$ has the same distribution as $\sum_{i \geqslant 1} Q_{i} W_{i-1}$. Therefore, we will derive the proposition from Theorem 4.3.1. So, we need to check that (4.3.1) and (4.3.2) hold. Referring to (4.3.1), we set $p=j-k$. Clearly $p+k$ is at most $m$. Moreover, define

$$
\rho=\frac{1}{2} \wedge \frac{\alpha}{\alpha+\omega}, \quad \text { and } \quad q=\left\lfloor\frac{\alpha+m}{\gamma \rho}+1\right\rfloor .
$$

Furthermore, set $s=\gamma \rho q$. Note that $t^{-s}=o\left(t^{-m} \bar{F}(t)\right)$ at infinity, for $s$ is more than $m+\alpha$.

Applying Hölder's inequality, we bound the expectation involved in (4.3.1) by

$$
\left(E|W|_{1}^{2 p}|W|_{k}^{2 k}\right)^{1 / 2}\left(E N_{\alpha, \gamma, \omega}(W)^{2 s}\right)^{1 / 2} t^{-s} .
$$

Now,

$$
|W|_{1}^{2 p}|W|_{k}^{2 k}=(2 p) ! 2 ! \sum_{n_{1}<\cdots<n_{2 p+2}} W_{n_{1}} \cdots W_{n_{2 p}} W_{n_{2 p+1}}^{k} W_{n_{2 p+2}}^{k}
$$

+ other terms where $n_{i}=n_{j}$ for some distinct $i$ and $j$.

In all the terms in the right hand side of (4.8.8), the $M_{i}$ 's are raised to a power at most $2(p+k)$, which is at most $2 m$. Concerning the first sum on the right hand side,

$$
\begin{aligned}
& E W_{n_{1}} \cdots W_{n_{2 p}} W_{n_{2 p+1}}^{k} W_{n_{2 p+2}}^{k} \\
& \quad=E W_{n_{1}}^{2 p+2 k}\left(\frac{W_{n_{2}}}{W_{n_{1}}}\right)^{2 p-1+2 k} \cdots\left(\frac{W_{n_{2 p+1}}}{W_{n_{2 p}}}\right)^{2 k}\left(\frac{W_{n_{2 p+2}}}{W_{n_{2 p+1}}}\right)^{k} \\
& =\mu_{H, 2 p+2 k}^{n_{1}} \mu_{H, 2 p-1+2 k}^{n_{2}-n_{1}} \cdots \mu_{H, 2 k}^{n_{2 p+1}-n_{2 p}} \mu_{H, k}^{n_{2 p+2}-n_{2 p+1}}
\end{aligned}
$$

By Lyapounov's inequality, for $j$ at most $2 m$, the inequality $\mu_{H, j} \leqslant$ $\mu_{H, 2 m}^{j / 2 m}$ holds. Therefore, (4.8.9) is at most $\mu_{H, 2 m}$ at the power

$$
\begin{aligned}
\frac{1}{2 m}\left((2 p+2 k) n_{1}+\right. & (2 p-1+2 k)\left(n_{2}-n_{1}\right)+\cdots \\
& \left.+2 k\left(n_{2 p+1}-n_{2 p}\right)+k\left(n_{2 p+2}-n_{2 p+1}\right)\right) .
\end{aligned}
$$

This exponent is at least

$$
\frac{1}{2 m}\left(k n_{1}+k\left(n_{2}-n_{1}\right)+\cdots+k\left(n_{2 p+2}-n_{2 p+1}\right)\right)=\frac{k n_{2 p+2}}{2 m} .
$$


Our moment assumption and Lyapounov's inequality imply that $\mu_{H, 2 m}$ is less than 1. Consequently, (4.8.9) is at most $\mu_{H, 2 m}^{k n_{2 p+2} / 2 m}$. For $n_{2 p+2}$ fixed, there are at most $n_{2 p+2}^{2 p+1}$ integers $n_{1}, \ldots, n_{2 p+1}$ less than $n_{2 p+2}$. Therefore, the first sum in the right hand side of (4.8.8) is at most

$$
\sum_{n \geqslant 1} n^{2 p+1}\left(\mu_{H, m}^{k / 2 m}\right)^{n}
$$

which is finite.

The other sums in the right hand side of (4.8.8) are similarly shown to be finite.

So, to check (4.3.1) it remains to prove that $N_{\alpha, \gamma, \omega}(W)^{2 s}$ has finite expectation. It suffices to show that $E|W|_{\gamma \rho}^{2 s}$ and $E|W|_{\infty}^{2 s}$ are finite.

We have

$$
E|W|_{\gamma \rho}^{2 s}=E\left(\sum_{i \geqslant 1} W_{i}^{\gamma \rho}\right)^{2 q}=\sum_{i_{1}, \ldots, i_{2 q}} W_{i_{1}}^{\gamma \rho} \cdots W_{i_{2 q}}^{\gamma \rho} .
$$

Again, this sum involves $M_{i}$ 's raised at power at most $2 q \gamma \rho$, that is $2 s$, which is less than $2(\alpha+m+\gamma \rho)$, which is less than $2(\alpha+m+1)$. By assumption, $\mu_{H, 2(\alpha+m+1)}$ is less than 1 , and the same argument as before shows that $E|W|_{\gamma \rho}^{2 s}$ is finite.

Finally, note that

$$
\begin{aligned}
P\left\{|W|_{\infty}^{2 s} \geqslant t\right\} & \leqslant \sum_{i \geqslant 1} P\left\{W_{i} \geqslant t^{1 / 2 s}\right\} \\
& \leqslant \sum_{i \geqslant 1} t^{-(\alpha+m+1) / s} E W_{i}^{2(\alpha+m+1)} \\
& \leqslant t^{-(\alpha+m+1) / s}\left(1-\mu_{H, 2(\alpha+m+1)}\right)^{-1}
\end{aligned}
$$

Since $(\alpha+m+1) / s$ is more than 1 , this shows that $|W|_{\infty}^{2 s}$ has finite expectation.

Finally, it is now straigtfoward to check that (4.3.2) holds.

Let us now give an explicit example. Its purpose is to show that it is now easy to obtain the tail expansion, at least when the assumptions of Proposition 4.8.1 are satisfied. So for our example, take $H$ to be the exponential distribution function of mean $\theta$,

$$
H(t)=1-e^{-t / \theta}, \quad t \geqslant 0,
$$


and $K$ to be the Pareto distribution

$$
K(t)=1-(1+t)^{-\alpha}, \quad t \geqslant 0 .
$$

To check the assumptions of Proposition 4.8.1, we calculate

$$
E M^{\lambda}=\int x^{\lambda} \theta^{-1} e^{-x / \theta} \mathrm{d} x=\theta^{\lambda} \Gamma(1+\lambda)
$$

Therefore, the condition of Proposition 4.8.1 is simply

$$
\theta \leqslant \Gamma(2(\alpha+m)+3))^{-1 / 2(\alpha+m+1)}
$$

It is natural to consider the asymptotic scale $e_{i}(t)=t^{-\alpha-i}$, with $i$ positive and less than $\alpha$. We will derive only two terms, again not because of the difficulty of getting more, but for the space of writing the coefficients. So we assume $\alpha$ larger that 1 and derive a two terms expansion for the solution of (4.8.5).

In the chosen scale, $p_{\bar{K}}=(1-\alpha)^{\mathrm{t}}$. As we have seen in section 3.2 , the matrix representing the derivative is defined by $\mathcal{D} e_{i}=$ $-(\alpha+i) e_{i+1}$, that is

$$
\mathcal{D}=\left(\begin{array}{cc}
0 & 0 \\
-\alpha & 0
\end{array}\right)
$$

The matrix representing the multiplication is

$$
\mathcal{M}_{c}=c^{\alpha}\left(\begin{array}{ll}
1 & 0 \\
0 & c
\end{array}\right)
$$

The first moment of the Pareto distribution is $\mu_{K, 1}=1 /(\alpha-1)$ while that of the exponential distribution is $\mu_{H, 1}=\theta$. So the moment equation is

$$
\mu_{F, 1}=\mu_{K, 1}+\mu_{H, 1} \mu_{F, 1}=(\alpha-1)^{-1}+\theta \mu_{F, 1} .
$$

Therefore,

$$
\mu_{F, 1}=1 /(1-\theta)(\alpha-1) .
$$

We then evaluate the matrices involved in (4.8.6). So,

$$
\mathcal{L}_{K}=\left(\begin{array}{cc}
1 & 0 \\
\alpha /(\alpha-1) & 1
\end{array}\right)
$$


and

$$
\begin{aligned}
\int \mathcal{M}_{x} \mathrm{~d} H(x) & =\int \operatorname{diag}\left(x^{\alpha}, x^{\alpha+1}\right) \theta^{-1} e^{-x / \theta} \mathrm{d} x \\
& =\theta^{\alpha} \Gamma(\alpha+1)\left(\begin{array}{cc}
1 & 0 \\
0 & \theta(\alpha+1)
\end{array}\right)
\end{aligned}
$$

After some elementary calculation,

$$
\mathcal{L}_{H * F}^{\mathrm{M}}=\left(\begin{array}{cc}
1 & 0 \\
\theta \alpha /(1-\theta)(\alpha-1) & 1
\end{array}\right)
$$

Now, applying formula (4.8.6), we proved that $\bar{F}$ has an asymptotic expansion $\bar{F} \sim p_{\bar{F}, 0} e_{0}+p_{\bar{F}, 1} e_{1}$ with

$$
p_{\bar{F}}=\left(\begin{array}{c}
\frac{1}{1-\theta^{\alpha} \Gamma(\alpha+1)} \\
\alpha \frac{\theta^{\alpha} \Gamma(\alpha+1)(\theta-\alpha+\theta \alpha)+\alpha-1-\theta \alpha}{(1-\alpha)(1-\theta)\left(1-\theta^{\alpha} \Gamma(\alpha+1)\right)\left(1-\theta^{\alpha+1} \Gamma(\alpha+2)\right)}
\end{array}\right) .
$$

To conclude this section, we mention that there are other parts of mathematics where regular variation plays an important role is differential equations; see for instance Marić and Tomić (1977), Omey (1981) and Marić (2000). Since the asymptotically smooth functions of fixed order can be differentiated a certain number of times, they form a natural class to use in differential equations. The general philosophy of this paper could be applied to some of these equations. Moreover, because of Theorem 2.3.1 it is possible to obtain asymptotic expansion for some integro-differential equations involving convolutions, Mellin transforms, multiplication by a function, nonlinearity due to taking powers of the unknown function and similar features for which we can find a stable asymptotic scale.

\section{Proof in the positive case.}

In this section, we prove Theorem 2.3.1 under some extra assumptions. Throughout this section, we suppose that $F^{(k)}$ is bounded and Lebesgue integrable over the positive half line. More importantly, we also suppose that both the $c_{i}$ 's and the $X_{i}$ 's are nonnegative. Since $\mathbb{Z}$ and $\mathbb{N}^{*}$ are in bijection, there is no loss of generality to index our sequence $\left(c_{i}\right)$ by $\mathbb{N}^{*}$, which we will do throughout the proof. In the first subsection, we derive an expression for convolutions which will be suitable for our analysis. In the second subsection, we outline the 
proof. The third subsection contains some basic facts about regularly varying functions and tail estimates. Subsection 4 contains a key estimate. Then we prove some basic lemmas (subsection 5) and finally conclude the proof by induction.

There will be many results during the proof which state that some term $A$ say is at most some term $B$. In some cases, nothing prevents a priori $B$ to be infinite. However, at the end, we will see that all the upper bounds are indeed finite and prove the theorem.

During the proof, $M$ denotes a generic constant which may change from place to place. This constant 'depends' only on $F$ and $\omega$. Also several of our lemmas below have conclusions which hold for all sufficiently large reals. The bounds above which these conclusions hold are denoted by $t_{i}$. These $t_{i}$ 's only depend on $F$ and $\omega$.

Since we consider nonnegative $c_{i}$ 's thoughout this section, we assume without any loss of generality that the sequence $\left(c_{n}\right)_{n \geqslant 1}$ is nonincreasing. It is also understood that except in subsection 5.1, all distributions in the current section are supported by the nonnegative half line. Recall the notation $C_{s}$ for the sum of the $c_{i}^{s}$, that is, in this section, $C_{s}=\sum_{i \geqslant 1} c_{i}^{s}$.

Note also that since $\omega$ is at least 1 , the function $\bar{F}$ is normalized regularly varying.

5.1. Decomposition of the convolution into integral and multiplication operators. Let $K$ be a distribution function and $h$ be a function integrable with respect to the measure $\mathrm{d} K$. For any $\eta$ between 0 and 1 , define the operator

$$
T_{K, \eta} h(t)=\int_{-\infty}^{\eta t} h(t-x) \mathrm{d} K(x) .
$$

Recall that for any positive real number $c$, we defined the multiplication operator on functions

$$
M_{c} h(t)=h(t / c) .
$$

It is then natural to define, when possible,

$$
M_{0} h(t)= \begin{cases}\lim _{s \rightarrow+\infty} h(s) & \text { if } t \geqslant 0 \\ \lim _{s \rightarrow-\infty} h(s) & \text { if } t<0 .\end{cases}
$$

Note that if a random variable $X$ has distribution function $F$, then $c X$ has distribution function $M_{c} F$. Moreover, for any $c$ nonnegative, $\overline{M_{c} F}=M_{c} \bar{F}$. 
These operators allow us to write convolutions and their derivative in a way that will be suitable for our analysis.

Proposition 5.1.1. Let $F$ and $G$ be two distribution functions. For any $\eta$ between 0 and 1 ,

$$
\overline{F \star G}=T_{G, 1-\eta} \bar{F}+T_{F, \eta} \bar{G}+M_{1 / \eta} \bar{F} M_{1 /(1-\eta)} \bar{G} .
$$

Let $k$ be a positive integer. If $F$ and $G$ are $k$ times differentiable,

$\overline{F \star G}^{(k)}=T_{G, 1-\eta} \bar{F}^{(k)}+T_{F, \eta} \bar{G}^{(k)}-\sum_{1 \leqslant i \leqslant k-1} M_{1 / \eta} \bar{F}^{(i)} M_{1 /(1-\eta)} \bar{G}^{(k-i)}$.

When $k=1$, the sum $\sum_{1 \leqslant i \leqslant 0}$ in the proposition must be read as 0. Equivalently,

$$
(F \star G)^{\prime}=T_{G, 1-\eta} F^{\prime}+T_{F, \eta} G^{\prime} .
$$

The proof of the proposition will be based on a lemma describing the behavior of the operator $T_{K, \eta}$ and $M_{c}$ under differentiation.

Lemma 5.1.2. If $K$ is a differentiable distribution function and if $h$ is a differentiable function, then

$$
\left(T_{K, \eta} h\right)^{\prime}=T_{K, \eta} h^{\prime}+\eta M_{1 /(1-\eta)} h M_{1 / \eta} K^{\prime}
$$

and

$$
\left(M_{c} h\right)^{\prime}=c^{-1} M_{c} h^{\prime}
$$

Proof. If $K$ is differentiable, then

$$
T_{K, \eta} h(t)=\int_{-\infty}^{\eta t} h(t-x) K^{\prime}(x) \mathrm{d} x
$$

The chain rule yields

$$
\frac{\mathrm{d}}{\mathrm{d} t} T_{K, \eta} h(t)=\int_{-\infty}^{\eta t} h^{\prime}(t-x) K^{\prime}(x) \mathrm{d} x+\eta h(t(1-\eta)) K^{\prime}(\eta t)
$$

which is the first statement. The second one is immediate. 
Proof (of the Proposition). The tail of the convolution $F * G$ is

$$
\begin{aligned}
\overline{F \star G}(t) & =\int_{-\infty}^{\infty} \bar{F}(t-y) \mathrm{d} G(y) \\
& =\int_{-\infty}^{t(1-\eta)} \bar{F}(t-y) \mathrm{d} G(y)+\int_{t(1-\eta)}^{\infty} \bar{F}(t-y) \mathrm{d} G(y)
\end{aligned}
$$

We integrate by parts and make a change of variable to obtain

$$
\begin{aligned}
\int_{t(1-\eta)}^{\infty} & \bar{F}(t-y) \mathrm{d} G(y) \\
= & {[\bar{F}(t-y)(G(y)-1)]_{t(1-\eta)}^{\infty}+\int_{t(1-\eta)}^{\infty} \bar{G}(y) \mathrm{d} \bar{F}(t-y) } \\
= & \bar{F}(t \eta) \bar{G}(t(1-\eta))+\int_{-\infty}^{t \eta} \bar{G}(t-y) \mathrm{d} F(y) .
\end{aligned}
$$

Combined with (5.1.1), we obtain the first assertion of the proposition.

To prove the second assertion, we proceed by induction, starting to prove the result for $k=1$. Using the lemma and differentiating the expression for $\overline{F \star G}$, we see that

$$
\begin{aligned}
\overline{F \star G^{\prime}=} T_{G, 1-\eta} \bar{F}^{\prime}+T_{F, \eta} \bar{G}^{\prime}+(1-\eta) M_{1 / \eta} \bar{F} M_{1 /(1-\eta)} G^{\prime} \\
+\eta M_{1 /(1-\eta)} \bar{G} M_{1 / \eta} F^{\prime}+\eta M_{1 / \eta} \bar{F}^{\prime} M_{1 /(1-\eta)} \bar{G} \\
+(1-\eta) M_{1 / \eta} \bar{F} M_{1 /(1-\eta)} \bar{G}^{\prime}
\end{aligned}
$$

Since $\bar{F}^{\prime}=-F^{\prime}$, we obtain $\overline{F \star G^{\prime}}=T_{G, 1-\eta} \bar{F}^{\prime}+T_{F, \eta} \bar{G}^{\prime}$.

Assume now that the relation holds for any integer up to $k-1$. Using the lemma,

$$
\begin{aligned}
\left(\overline{F \star G}^{(k-1)}\right)^{\prime}= & T_{G, 1-\eta} \bar{F}^{(k)}+T_{F, \eta} \bar{G}^{(k)} \\
& +(1-\eta) M_{1 / \eta} \bar{F}^{(k-1)} M_{1 /(1-\eta)} G^{\prime} \\
& +\eta M_{1 /(1-\eta)} \bar{G}^{(k-1)} M_{1 / \eta} F^{\prime} \\
& -\sum_{1 \leqslant i \leqslant k-2} \eta M_{1 / \eta} \bar{F}^{(i+1)} M_{1 /(1-\eta)} \bar{G}^{(k-1-i)} \\
& -\sum_{1 \leqslant i \leqslant k-2}(1-\eta) M_{1 / \eta} \bar{F}^{(i)} M_{1 /(1-\eta)} \bar{G}^{(k-i)},
\end{aligned}
$$


which after collecting the terms give the proper expression for $\overline{F \star G}^{(k)}$.

5.2. Organizing the proof. The proof goes essentially by induction on $k$, and for fixed $k$ by induction on $n$. We write $G_{n}$ the distribution funciton of $\sum_{1 \leqslant i \leqslant n} c_{i} X_{i}$ and $G$ that of $\langle c, X\rangle$. We also write $F_{i}$ for $M_{c_{i}} F$, that is for the distribution function of $c_{i} X_{i}$. Hence, $G_{n}=F_{1} \star \cdots \star F_{n}$.

We also define a paramater $\rho$ in $(0,1)$, which will be chosen at the end of the proof. We set $d_{n}=c_{n}^{\rho}$.

Applying Proposition 5.1.1, we see that

$$
\begin{aligned}
\bar{G}_{n}^{(k)}=T_{G_{n-1}, 1-d_{n}} \bar{F}_{n}^{(k)} & +T_{F_{n}, d_{n}} \bar{G}_{n-1}^{(k)} \\
& -\sum_{1 \leqslant i \leqslant k-1} M_{1 / d_{n}} \bar{F}_{n}^{(i)} M_{1 /\left(1-d_{n}\right)} \bar{G}_{n-1}^{(k-i)} .
\end{aligned}
$$

Let us write $\mathcal{A}_{m} \bar{G}_{n}^{(k)}$ the $m$ terms asymptotic expansion of $\bar{G}_{n}^{(k)}$ - or, more precisely, for the time being, the candidate for this asymptotic expansion -

$$
\mathcal{A}_{m} \bar{G}_{n}^{(k)}=\sum_{1 \leqslant i \leqslant n} L_{G_{n} \mapsto F_{i}, m} \bar{F}_{i}^{(k)} .
$$

The ' $\mathcal{A}_{m}$ ' in this formula is not an operator; it is aimed as a short hand for ' $m$-terms approximation of' and merely to help the memory. Sometimes we will omit the subscript $m$, writing simply $\mathcal{A}$.

We will approximate $T_{G_{n-1}, 1-d_{n}}$ by $L_{G_{n-1}}$ (Theorem 5.4.1) and $T_{F_{n}, d_{n}}$ by $L_{F_{n}}$. Thus, we expect an approximation of $\bar{G}_{n}^{(k)}$ by $L_{G_{n-1}} \bar{F}_{n}^{(k)}+L_{F_{n}} \bar{G}_{n-1}^{(k)}$. Since $\bar{G}_{n-1}^{(k)}$ is close to its asymptotic expansion, we also hope to approximate $L_{F_{n}} \bar{G}_{n-1}^{(k)}$ by $L_{F_{n}} \mathcal{A} \bar{G}_{n-1}^{(k)}$. But

$$
L_{F_{n}} \mathcal{A} \bar{G}_{n-1}^{(k)}=\sum_{1 \leqslant i \leqslant n-1} L_{F_{n}} L_{G_{n-1} \natural F_{i}} \bar{F}_{i}^{(k)} .
$$

Now, $L_{F_{n}} L_{G_{n-1} \natural F_{i}}$ is not $L_{F_{n} \star\left(G_{n-1} \natural F_{i}\right)}$ (the latter being $L_{G_{n} \sharp F_{i}}$ ); however, these two operators are equal up to differential operators involving $\mathrm{D}^{m+1+i}$ 's for $i$ nonnegative. Since $\bar{F}_{i}^{(k+l)} \asymp \mathrm{Id}^{-l} \bar{F}_{i}^{(k)}$ when $\bar{F}_{i}^{(k+l)}$ is regularly varying, we expect that

$$
L_{F_{n}} \mathcal{A} \bar{G}_{n-1}^{(k)} \sim \sum_{1 \leqslant i \leqslant n-1} L_{G_{n} \natural F_{i}} \bar{F}_{i}^{(k)} .
$$


Hence, we should obtain

$$
\bar{G}_{n}^{(k)} \sim L_{G_{n-1}} \bar{F}_{n}^{(k)}+\sum_{1 \leqslant i \leqslant n-1} L_{G_{n} \sharp F_{i}} \bar{F}_{i}^{(k)},
$$

which is equal to $\mathcal{A} \bar{G}_{n}^{(k)}$. Thus, assuming that $\bar{G}_{n-1}^{(k)} \sim \mathcal{A} \bar{G}_{n-1}^{(k)}$, we can expect to prove $\bar{G}_{n}^{(k)} \sim \mathcal{A} \bar{G}_{n}^{(k)}$, and build this way a proof by induction on $n$. In any case, this derivation shows that

$$
\begin{aligned}
\bar{G}_{n}^{(k)}-\mathcal{A} \bar{G}_{n}^{(k)}= & \left(T_{G_{n-1}, 1-d_{n}}-L_{G_{n-1}}\right) \bar{F}_{n}^{(k)} \\
& +T_{F_{n}, d_{n}}\left(\bar{G}_{n-1}^{(k)}-\mathcal{A} \bar{G}_{n-1}^{(k)}\right) \\
& +\left(T_{F_{n}, d_{n}} \mathcal{A} \bar{G}_{n-1}^{(k)}-\sum_{1 \leqslant i \leqslant n-1} L_{G_{n} \natural F_{i}} \bar{F}_{i}^{(k)}\right) \\
& -\sum_{1 \leqslant j \leqslant k-1} M_{1 / d_{n}} \bar{F}_{n}^{(j)} M_{1 / 1-d_{n}} \bar{G}_{n-1}^{(k-j)} .
\end{aligned}
$$

In the right hand side of this equality, we control $\bar{F}_{n}^{(k)}$ by assumptions on $\bar{F}$ and the difference $T_{G_{n-1}, 1-d_{n}}-L_{G_{n-1}}$ by Theorem 5.4.1. Since $\mathcal{A} \bar{G}_{n-1}^{(k)}$ is expressed in terms of functions $\bar{F}_{i}$ 's, hence, ultimately in terms of $\bar{F}$, it is an explicit term on which we can work. The only term on which we do not have a rather direct control is $T_{F_{n}, d_{n}}\left(\bar{G}_{n-1}^{(k)}-\mathcal{A} \bar{G}_{n-1}^{(k)}\right)$. Up to a shift of index $n$, it is related to the left hand side of (5.2.1), provided we can control the operator norm of $T_{F_{n}, d_{n}}$. This will allow us to prove the asymptotic expansion $\bar{G}_{n}^{(k)} \sim \mathcal{A}_{m} \bar{G}_{n}^{(k)}$ is valid uniformly in $n$.

For the induction and the estimates to be written in a manageable form, we use a pseudo-semi-norm to control the remainder terms. For any function $h$ on the nonnegative half line, we define

$$
|h|_{m, \tau}=\sup _{t \geqslant \tau} t^{m}|h(t)| / \bar{F}(t) .
$$

The choice of this pseudo-semi-norm is motivated by the fact that $\lim _{\tau \rightarrow \infty}|h|_{m, \tau}=0$ is equivalent to $h(t)=o\left(t^{-m} \bar{F}(t)\right)$ as $t$ tends to infinity. Therefore, proving an asymptotic equivalence up to a $t^{-m} \bar{F}(t)$ term amounts to prove that the pseudo-semi-norm $|\cdot|_{m, \tau}$ of some function tends to 0 as $\tau$ tends to infinity.

5.3. Regular variation and basic tail estimates. In this subsection, we collect some facts on regular variation and on the distribution of $\langle c, X\rangle$. Our first lemma sandwiches the tail of $\langle c, X\rangle$. 
Lemma 5.3.1. If $C_{\rho} \leqslant 1$, then

$$
M_{c_{1}} \bar{F} \leqslant \bar{G}_{n} \leqslant \bar{G} \leqslant \sum_{n \geqslant 1} M_{c_{n}^{1-\rho}} \bar{F}
$$

Moreover, let $\epsilon$ be a positive real number. There exists $t_{3}$ such that for any $t$ larger than $t_{3}$,

$$
\bar{G}(t) \leqslant C_{\alpha(1-\rho)-\epsilon} \bar{F}(t) .
$$

Proof. The first two bounds follow from the inequalities

$$
c_{1} X_{1} \leqslant c_{1} X_{1}+\cdots+c_{n} X_{n} \leqslant\langle c, X\rangle \text {. }
$$

Next, if $c_{n}^{1-\rho} X_{n} \leqslant t$ for all $n$, then

$$
\sum_{n \geqslant 1} c_{n} X_{n} \leqslant t \sum_{n \geqslant 1} c_{n}^{\rho}=t C_{\rho} \leqslant t
$$

Therefore,

$$
P\{\langle c, X\rangle>t\} \leqslant P\left\{\cup_{n \geqslant 1}\left\{c_{n}^{1-\rho} X_{n}>t\right\}\right\}=\sum_{n \geqslant 1} P\left\{c_{n}^{1-\rho} X_{n}>t\right\},
$$

which is the last inequality in the first statement. This inequality asserts that $\bar{G}(t)$ is at most $\sum_{n \geqslant 1} \bar{F}\left(t / c_{n}^{1-\rho}\right)$. Since $C_{\rho}$ is at most 1 , so are all the $c_{i}$ 's. Apply Lemma 2.2.4 with $g=\bar{F}, \delta=\epsilon /(1-\rho)$ and $\lambda=c_{n}^{\rho-1}$ to obtain the second statement.

This lemma will be useful when combined with the next analytical result.

Lemma 5.3.2. Let $g, h$ be two nonincreasing functions of bounded variations, such that $g \leqslant h$ and

$$
\lim _{t \rightarrow \infty} g(t)=\lim _{t \rightarrow \infty} h(t)=0 .
$$

Let $f$ be a nonnegative nondecreasing and right continuous function on $\mathbb{R}^{+}$. Then

$$
-\int f \mathrm{~d} g \leqslant-\int f \mathrm{~d} h
$$


Proof. Define the left continuous inverse of $f$ by

$$
f^{\leftarrow}(u)=\inf \{x: f(x) \geqslant u\}
$$

Observe that $f(x) \geqslant u$ if and only if $f^{\leftarrow}(u) \leqslant x$. We use the FubiniTonnelli theorem to write

$$
\begin{aligned}
\int_{0}^{\infty} f \mathrm{~d} g & =\int_{0}^{\infty} \int_{0}^{\infty} \mathbb{I}\{u \leqslant f(x)\} \mathrm{d} u \mathrm{~d} g(x) \\
& =\int_{0}^{\infty} \int_{0}^{\infty} \mathbb{I}\left\{x \geqslant f^{\leftarrow}(u)\right\} \mathrm{d} g(x) \mathrm{d} u \\
& =-\int_{0}^{\infty} g \circ f^{\leftarrow}(u) \mathrm{d} u .
\end{aligned}
$$

The result follows from the inequality $g \circ f^{\leftarrow} \leqslant h \circ f^{\leftarrow}$.

Since $\bar{G}, \bar{G}_{n}, \bar{F}_{i}$ and $\bar{F}$ are of the same order, so are their truncated means $\int_{t}^{\infty} x^{k} \mathrm{~d} K(x)$ for $K=\bar{G}, \bar{G}_{n}, \bar{F}_{i}$ and $\bar{F}$. Order is related to asymptotic behavior, and because we will need to have estimates that are uniform over $n$, we need a quantitative statement comparing truncated means. The following will do for our purpose and may be useful in other contexts.

Lemma 5.3.3. Let $\bar{F}$ be a normalized regularly varying function of index $-\alpha$. Let $\delta$ be a positive number. There exists $t_{4}$ such that for any $0<u \leqslant v$, any $t \geqslant t_{4}$, any integer $k<\alpha$,

$$
\int_{t v}^{\infty} x^{k} \mathrm{~d} M_{u} F(x) \leqslant \frac{2 \alpha}{\alpha-k} v^{k}\left(\frac{u}{v}\right)^{\alpha-\delta} t^{k} \bar{F}(t) .
$$

Proof. A change of variable yields

$$
\int_{t v}^{\infty} x^{k} \mathrm{~d} M_{u} F(x)=u^{k} \int_{t v / u}^{\infty} x^{k} \mathrm{~d} F(x) .
$$

By Karamata's theorem for Stieltjes integrals (Bingham, Goldie, Teugels, 1989, Theorem 1.6.5), there exists a $t_{4}^{\prime}$ such that for $t$ more than $t_{4}^{\prime}$

$$
\int_{t}^{\infty} x^{k} \mathrm{~d} F(x) \leqslant \frac{2 \alpha}{\alpha-k} t^{k} \bar{F}(t)
$$


This $t_{4}^{\prime}$ may be taken independent of $k$ since there is only a finite number of integers less than $\alpha$. Since $v / u \geqslant 1$, this implies that for $t$ at least $t_{4}^{\prime}$,

$$
\int_{t v}^{\infty} x^{k} \mathrm{~d} M_{u} F(x) \leqslant \frac{2 \alpha}{\alpha-k} v^{k} t^{k} \bar{F}(t v / u)
$$

By Lemma 2.2.4, if $t \geqslant t_{2}$, we have $\bar{F}(t v / u) \leqslant(u / v)^{\alpha-\delta} \bar{F}(t)$. Take $t_{4}=t_{2} \vee t_{4}^{\prime}$ to conclude.

Lemma 5.3.4. Let $\bar{F}$ be a normalized regularly varying function with index $-\alpha$. Let $\epsilon$ be a positive number. There exists $t_{5}$ such that for any $t \geqslant t_{5}$, any $n \geqslant 1$, any sequence $\left(c_{n}\right)_{n \geqslant 1}$ with $C_{\rho} \leqslant 1$, and any $k<\alpha$,

$$
\int_{t}^{\infty} x^{k} \mathrm{~d} G_{n}(x) \leqslant \int_{t}^{\infty} x^{k} \mathrm{~d} G(x) \leqslant \frac{2 \alpha}{\alpha-k} C_{\alpha(1-\rho)-\epsilon} t^{k} \bar{F}(t) .
$$

Proof. Combining Lemmas 5.3.1 and 5.3.2, we have

$$
\int_{t}^{\infty} x^{k} \mathrm{~d} G_{n}(x) \leqslant \int_{t}^{\infty} x^{k} \mathrm{~d} G(x) \leqslant \sum_{n \geqslant 1} \int_{t}^{\infty} x^{k} \mathrm{~d} M_{c_{n}^{1-\rho}} F(x),
$$

where we used that $C_{\rho} \leqslant 1$ in the last inequality. Since $C_{\rho}$ is at most 1 , so are all the $c_{n}$ 's. We apply Lemma 5.3 .3 with $\delta=\epsilon /(1-\rho)$ to obtain

$$
\int_{t}^{\infty} x^{k} \mathrm{~d} M_{c_{n}^{1-\rho}} F(x) \leqslant \frac{2 \alpha}{\alpha-k} c_{n}^{(1-\rho)(\alpha-\delta)} t^{k} \bar{F}(t)
$$

for $t$ at least $t_{4}$

We will also use the following bound on the moment of $G$.

Lemma 5.3.5. Assume $C_{\rho}$ is at most 1 . For any $s$ at least 1 ,

$$
\mu_{G, s} \leqslant C_{s(1-\rho)} \mu_{F, s}
$$

Proof. Write

$$
\mu_{G, s}=s \int_{0}^{\infty} x^{s-1} \bar{G}(x) \mathrm{d} x
$$


Apply Lemma 5.3.1 to obtain that the moment of order $s$ of $G$ is at most

$$
\begin{aligned}
\sum_{n \geqslant 1} \int_{0}^{\infty} s x^{s-1} M_{c_{n}^{1-\rho}} \bar{F}(x) \mathrm{d} x & =\sum_{n \geqslant 1} \int_{0}^{\infty}\left(c_{n}^{1-\rho}\right)^{s} x^{s} \mathrm{~d} F(x) \\
& =C_{s(1-\rho)} \mu_{F, s} .
\end{aligned}
$$

5.4. The fundamental estimate. The origin of our asymptotic expansions is the following estimate, whose proof is almost deceptively simple.

Theorem 5.4.1. Let $m$ be a positive integer and let $r$ be in $[0,1)$. Furthermore, let $K$ be a distribution function on the nonnegative half line, whose $m$-th moment is finite. If $h$ is smoothly varying of order $m+r$, then

$$
\begin{aligned}
\left|\left(T_{K, \eta}-L_{K, m}\right) h\right|(t) \leqslant & \sum_{0 \leqslant j \leqslant m} \frac{\left|h^{(j)}(t)\right|}{j !} \int_{\eta t}^{\infty} x^{j} \mathrm{~d} K(x) \\
& +\frac{\left|h^{(m)}(t)\right|}{t^{r} m !} \int_{0}^{\eta t} \bar{\Delta}_{t, x / t}^{r}\left(h^{(m)}\right) x^{m+r} \mathrm{~d} K(x) .
\end{aligned}
$$

Proof. The expression of $T_{K, \eta}$ and Proposition 2.2.5 show that

$$
\begin{aligned}
\mid T_{K, \eta} h(t) & -\int_{0}^{\eta t} \sum_{0 \leqslant j \leqslant m}(-1)^{j} \frac{x^{j}}{j !} h^{(j)}(t) \mathrm{d} K(x) \mid \\
& \leqslant \frac{\left|h^{(m)}(t)\right|}{t^{r} m !} \int_{0}^{\eta t} \bar{\Delta}_{t, x / t}^{r}\left(h^{(m)}\right) x^{m+r} \mathrm{~d} K(x) .
\end{aligned}
$$

Since

$$
\int_{0}^{\eta t}(-1)^{j} \frac{x^{j}}{j !} h^{(j)}(t) \mathrm{d} K(x)=\frac{(-1)^{j}}{j !}\left(\mu_{K, j}-\int_{\eta t}^{\infty} x^{j} \mathrm{~d} K(x)\right) \mathrm{D}^{j} h(t),
$$

the result follows from the triangle inequality.

We could also bound (5.4.1) by the simpler estimate

$$
t^{-r}\left|h^{(m)}(t)\right| \bar{\Delta}_{t, \eta}^{r}\left(h^{(m)}\right) \mu_{K, m+r} .
$$


Unfortunately, this is not good enough for our purpose.

Let us now explain why the estimate in the previous Theorem is useful. When $h$ is smoothly varying and has index $-\beta$, the term of smallest order in $L_{K, m} h$ is given by $h^{(m)}$, which is in $R V_{-\beta-m}$. If $\bar{K}$ is regularly varying with index $-\alpha$, Karamata's theorem for Stieltjes integrals (Bingham, Goldie and Teugels, 1989, Theorem 1.6.5) shows that the function $t \mapsto \int_{\eta t}^{\infty} x^{i} \mathrm{~d} K(x)$ is in $R V_{-\alpha+i}$. Thus, $h^{(i)}(t) \int_{\eta t}^{\infty} x^{i} \mathrm{~d} K(x)$ is regularly varying with index $-\alpha-\beta$; this index is smaller than $-\beta-m$ if $m<\alpha$. Thus, Theorem 5.4.1 proves that when $h$ is smoothly varying, $T_{K, \eta} h \sim L_{K, m} h$, and, moreover, gives an estimate of the error term of this asymptotic expansion.

5.5. Basic lemmas. The goal of this subsection is to give estimates for the first three terms of the right hand side of (5.2.1).

Our first lemma takes care of the term $\left(T_{G_{n-1}, 1-d_{n}}-L_{G_{n-1}, m}\right) \bar{F}_{n}^{(k)}$. It asserts that it is of smaller order than $t^{-m-k} \bar{F}(t)$, uniformly in $n$ and in some sequences $\left(c_{n}\right)_{n \geqslant 1}$. We write $v(t)$ a function such that $0 \leqslant v(t) \leqslant t$ for all nonnegative $t$, and $\lim _{t \rightarrow \infty} v(t)=+\infty$ while $\lim _{t \rightarrow \infty} v(t) / t=0$. We assume moreover that $v(t) / t$ is ultimately nonincreasing. For instance, $v(t)=t^{\kappa}$ with $0<\kappa<1$ will do.

Lemma 5.5.1. Let $\epsilon$ be a positive number. Assume that $\bar{F}$ is in $S R_{-\alpha, m+r+k}$ with $m$ an integer, $r$ in $[0,1)$ and $m+r$ smaller than $\alpha$. There exist $M$ and $t_{6}$ depending only on $F, m, k$ and $\epsilon$ such that whenever $C_{\rho} \vee C_{(1-\rho)(m+r)} \leqslant 1$, and whenever $d_{n} \leqslant 1 / 2$, for any $t$ at least $t_{6}$,

$$
\begin{aligned}
& \left|\left(T_{G_{n-1}, 1-d_{n}}-L_{G_{n-1}, m}\right) \bar{F}_{n}^{(k)}\right|_{k+m, t} \\
& \quad \leqslant M c_{n}^{\alpha-(\alpha+k+m) \rho-\epsilon}\left(\bar{\Delta}_{t, v(t) / t}^{r}\left(\bar{F}^{(k+m)}\right)+v(t)^{m+r} \bar{F} \circ v(t)\right) .
\end{aligned}
$$

Proof. Applying Theorem 5.4.1, we obtain for $t$ at least 1,

$$
\begin{aligned}
& \left|\left(T_{G_{n-1}, 1-d_{n}}-L_{G_{n-1}, m}\right) \bar{F}_{n}^{(k)}\right|(t) \\
& \quad \leqslant \sum_{0 \leqslant j \leqslant m}\left|\bar{F}_{n}^{(k+j)}(t)\right| \int_{t\left(1-d_{n}\right)}^{\infty} x^{j} \mathrm{~d} G_{n-1}(x) \\
& \quad+\left|\bar{F}_{n}^{(k+m)}(t)\right| \int_{0}^{t\left(1-d_{n}\right)} \bar{\Delta}_{t, x / t}^{r}\left(\bar{F}_{n}^{(k+m)}\right) x^{m+r} \mathrm{~d} G_{n-1}(x) .
\end{aligned}
$$


Since the $c_{i}$ 's are all at most 1 , it suffices to prove the result for $\epsilon$ small enough. Thus, we can assume that $m+r$ is at most $\alpha-\epsilon$. Let $\delta=\epsilon /(2-\rho)$. We can assume that $\epsilon$ is small enough so that $(1-\rho) \alpha-\delta$ is at least $(1-\rho)(m+r)$. Then, the sum $C_{\alpha(1-\rho)-\delta}$ is at most $C_{(1-\rho)(m+r)}$, hence at most 1. Combining Lemmas 2.2.3 and 5.3.4, we see that for $t\left(1-d_{n}\right) \geqslant t_{1} \vee t_{5}$,

$$
\begin{aligned}
\left|\bar{F}_{n}^{(k+j)}(t)\right| \int_{t\left(1-d_{n}\right)}^{\infty} x^{j} \mathrm{~d} G_{n-1} & \leqslant M c_{n}^{\alpha-\delta} t^{-k-j} \bar{F}(t) t^{j} \bar{F}(t) \\
& \leqslant M c_{n}^{\alpha-\delta} t^{-k} \bar{F}(t)^{2} .
\end{aligned}
$$

Applying Lemmas 2.2.3, 2.2.6, 5.3.1 and 5.3.2, we also have, for $t$ at least $t_{1}$ and $t_{2}$,

$$
\begin{aligned}
& \left|\bar{F}_{n}^{(k+m)}(t)\right| \int_{0}^{t\left(1-d_{n}\right)} \bar{\Delta}_{t, x / t}^{r}\left(\bar{F}_{n}^{(k+m)}\right) x^{m+r} \mathrm{~d} G_{n-1}(x) \\
& \quad \leqslant M c_{n}^{\alpha-\delta} t^{-k-m} \bar{F}(t) \int_{0}^{t\left(1-d_{n}\right)} \bar{\Delta}_{t / c_{n}, x / t}^{r}\left(\bar{F}^{(k+m)}\right) x^{m+r} \mathrm{~d} G(x) .
\end{aligned}
$$

We split the integral in this upper bound as one for $x$ between 0 and $v(t)$, and one between $v(t)$ and $t\left(1-d_{n}\right)$. We then have the easy bound

$$
\int_{0}^{v(t)} \bar{\Delta}_{t / c_{n}, x / t}^{r}\left(\bar{F}^{(k+m)}\right) x^{r+m} \mathrm{~d} G(x) \leqslant \bar{\Delta}_{t, v(t) / t}^{r}\left(\bar{F}^{(k+m)}\right) \mu_{G, m+r} .
$$

We use Lemma 5.3 .5 to bound $\mu_{G, m+r}$ by $C_{(m+r)(1-\rho)} \mu_{F, m+r}$, which is then at most $\mu_{F, m+r}$ under the assumptions of the lemma.

To bound the integral for $x$ between $v(t)$ and $t\left(1-d_{n}\right)$, we first bound $\bar{\Delta}_{t / c_{n}, x / t}^{r}\left(\bar{F}^{(k+m)}\right)$ by $\bar{\Delta}_{t / c_{n}, 1-d_{n}}^{r}\left(\bar{F}^{(k+m)}\right)$. The latter is at most

$\bar{\Delta}_{t / c_{n}, 1 / 2}^{r}\left(\bar{F}^{(k+m)}\right)+\sup _{s \geqslant t / c_{n}} \sup _{1 / 2<|y| \leqslant 1-d_{n}} y^{-r}\left(\left|\frac{\bar{F}^{(k+m)}(s(1-y))}{\bar{F}^{(k+m)}(s)}\right|+1\right)$.

If $s$ is at least $t / c_{n}$ and $y$ at most $1-d_{n}$, then $s(1-y)$ is at least $t d_{n} / c_{n}$, and therefore at least $t$. Thus, by the standard Potter bounds, there exists $t_{6}^{\prime}$ such that for $s$ at least $t_{6}^{\prime}$,

$$
\begin{aligned}
\left|\frac{\bar{F}^{(k+m)}(s(1-y))}{\bar{F}^{(k+m)}(s)}\right| & \leqslant 2\left((1-y)^{-\alpha-k-m-\delta} \vee(1-y)^{-\alpha-k-m+\delta}\right) \\
& \leqslant 2 d_{n}^{-\alpha-k-m-\delta} .
\end{aligned}
$$


Consequently,

$$
\begin{aligned}
& \int_{v(t)}^{t\left(1-d_{n}\right)} \bar{\Delta}_{t / c_{n}, x / t}^{r}\left(\bar{F}^{(k+m)}\right) x^{m+r} \mathrm{~d} G(x) \\
& \quad \leqslant M\left(\bar{\Delta}_{t / c_{n}, 1 / 2}^{r}\left(\bar{F}^{(k+m)}\right)+d_{n}^{-\alpha-k-m-\delta}+1\right) \int_{v(t)}^{\infty} x^{m+r} \mathrm{~d} G(x) .
\end{aligned}
$$

Since $d_{n}$ is smaller than $1 / 2$, we can simplify this upper bound into

$$
M d_{n}^{-\alpha-k-m-\epsilon} \int_{v(t)}^{\infty} x^{m+r} \mathrm{~d} G(x) .
$$

Applying Lemma 5.3.4, for $v(t)$ at least $t_{5}$, this last quantity is at most

$$
M d_{n}^{-\alpha-k-m-\delta} v(t)^{m+r} \bar{F} \circ v(t) .
$$

It follows that

$$
\begin{aligned}
& \int_{0}^{t\left(1-d_{n}\right)} \bar{\Delta}_{t / c_{n}, x / t}^{r}\left(\bar{F}^{(k+m)}\right) x^{m+r} \mathrm{~d} G(x) \\
& \quad \leqslant M \bar{\Delta}_{t, v(t) / t}^{r}\left(\bar{F}^{(k+m)}\right)+M d_{n}^{-\alpha-k-m-\delta} v(t)^{m+r} \bar{F} \circ v(t) .
\end{aligned}
$$

Combined with the other bounds, we obtain that the right hand side of (5.5.1) is at most

$$
\begin{aligned}
M c_{n}^{\alpha-\delta} t^{-k-m} \bar{F}(t)\left(t^{m} \bar{F}(t)\right. & +\bar{\Delta}_{t, v(t) / t}^{r}\left(\bar{F}^{(k+m)}\right) \\
& \left.+d_{n}^{-\alpha-k-m-\delta} v(t)^{m+r} \bar{F} \circ v(t)\right) .
\end{aligned}
$$

Since $m+r$ is smaller than $\alpha$, the function $s \mapsto s^{m+r} \bar{F}(s)$ is ultimately nonincreasing (Bingham, Goldie and Teugels, 1989, Theorem 1.5.3). Thus, for $t$ large enough, $t^{m} \bar{F}(t)$ is smaller than $2 v(t)^{m+r} \bar{F} \circ v(t)$. The proof is completed in noting that $d_{n}^{-\alpha-k-m-\delta}$ is at least 1.

Our next lemma shows that when $d_{n}$ is small, that is $n$ is large, then $T_{F_{n}, d_{n}}$ is very close to be a contraction.

Lemma 5.5.2. There exists some positive numbers $M$ and $t_{7}$ such that for any $t$ at least $t_{7}$, any positive $n$, any $k$, any $d_{n}$ smaller than $1 / 2$, any function $h$,

$$
\left|T_{F_{n}, d_{n}} h\right|_{k, t} \leqslant|h|_{k, t\left(1-d_{n}\right)}\left(1+M d_{n}\right) .
$$


Proof. A change of variable shows that

$$
T_{F_{n}, d_{n}} h(t)=\int_{0}^{t d_{n} / c_{n}} h\left(t-c_{n} x\right) \mathrm{d} F(x) .
$$

If $x$ is at most $t d_{n} / c_{n}$, then $t-c_{n} x$ is at least $t\left(1-d_{n}\right)$. Consequently,

$$
\left|h\left(t-c_{n} x\right)\right| \leqslant|h|_{k, t\left(1-d_{n}\right)} \frac{\bar{F}\left(t-c_{n} x\right)}{\left(t-c_{n} x\right)^{k}} \leqslant|h|_{k, t\left(1-d_{n}\right)} \frac{\bar{F}\left(t\left(1-d_{n}\right)\right)}{t^{k}\left(1-d_{n}\right)^{k}} .
$$

This implies

$$
\frac{t^{k}\left|T_{F_{n}, d_{n}} h(t)\right|}{\bar{F}(t)} \leqslant|h|_{k, t\left(1-d_{n}\right)} \frac{\bar{F}\left(t\left(1-d_{n}\right)\right)}{\bar{F}(t)}\left(1-d_{n}\right)^{-k} .
$$

Since $d_{n}$ is at most $1 / 2$, we can apply Lemma 2.2 .4 , and then bound $\left(1-d_{n}\right)^{-\alpha-k-\delta}$ by $1+M d_{n}$.

Remark. In proving Lemma 5.5.2, our improved Potter bound is crucial. With the usual one we could only get $A\left(1+M d_{n}\right)$ for some $A$ greater than 1 . This is not good enough for the inductions to come. Extensive attempts to prove our theorem under weaker assumptions suggest that the behavior of the function $c(\cdot)$ in the Karamta representation (see Bingham, Goldie and Teugels, 1989, Theorem 1.3.1) of $\bar{F}$ cannot be arbitrary for our asymptotic expansion to hold. In particular, the asymptotic behavior of $c(\cdot)$ plays a key role in the asymptotic expansion. This is already the case for a two terms expansion where the second term involves derivative; one should indeed remark that the absolute continuity of the function $c(\cdot)$ is equivalent to that of $\bar{F}$.

In particular, in connection with the second term in (5.2.1), we obtain the following estimate, considering $h=\bar{G}_{n-1}^{(k)}-\mathcal{A}_{m} \bar{G}_{n-1}^{(k)}$ in Lemma 5.5.2.

Corollary 5.5.3. There exist positive numbers $M$ and $t_{8}$ such that for any $t$ at least $t_{8}$ and any sequence $\left(c_{n}\right)_{n \geqslant 1}$ with $\left(d_{n}\right)_{n \geqslant 1}$ uniformly bounded by $1 / 2$, any $k$ and any $n$ at least 2 ,

$$
\begin{aligned}
\mid T_{F_{n}, d_{n}}\left(\bar{G}_{n-1}^{(k)}\right. & \left.-\mathcal{A}_{m} \bar{G}_{n-1}^{(k)}\right)\left.\right|_{k+m, t} \\
& \leqslant\left|\bar{G}_{n-1}^{(k)}-\mathcal{A}_{m} \bar{G}_{n-1}^{(k)}\right|_{k+m, t\left(1-d_{n}\right)}\left(1+M d_{n}\right) .
\end{aligned}
$$


This estimate shows the need to introduce the family of operators $T_{K, \eta}$ and not use $T_{K, 1 / 2}$ as in Barbe and McCormick (2005). Indeed, when doing the induction later, we see that the value of $t$ in the norm of the right hand side drops by a factor $1-d_{n}$. When applying this bound inductively, we will obtain something like

$$
\left|\bar{G}_{2}^{(k)}-\mathcal{A} \bar{G}_{2}^{(k)}\right|_{k+m, t \Pi_{1 \leqslant i \leqslant n}\left(1-d_{i}\right)} .
$$

If $\prod_{i \geqslant 1}\left(1-d_{i}\right)=0$, we will not be able to permute the limits as $n$ tends to infinity and $t$ tends to infinity. It is therefore essential that the series $\sum_{n \geqslant 1} d_{n}$ converges.

Our last lemma in this section will handle the third term in the right hand side of (5.2.1).

Lemma 5.5.4. Let $\epsilon$ be a positive number at most $\alpha-1$. Assume that $F$ is in $S R_{-\alpha, m+k+r}$. There exist positive $M$ and $t_{9}$ such that for any sequence $\left(c_{n}\right)_{n \geqslant 1}$ with $C_{\rho}$ at most 1 , any $t$ at least $t_{9}$ and any $n$ at least 2 ,

$$
\begin{aligned}
& \left|T_{F_{n}, d_{n}} \mathcal{A}_{m} \bar{G}_{n-1}^{(k)}-\sum_{1 \leqslant i \leqslant n-1} L_{G_{n} \natural F_{i}} \bar{F}_{i}^{(k)}\right|_{k+m, t} \\
& \leqslant M\left(c_{n}^{\alpha(1-\rho)-\epsilon}+c_{n}^{r} \bar{\Delta}_{t, d_{n}}^{r}\left(\bar{F}^{(k+m)}\right)\right) v(t)^{m+r} \bar{F} \circ v(t) \\
& \quad+M c_{n}^{r} \bar{\Delta}_{t, c_{n} v(t) / t}^{r}\left(\bar{F}^{(k+m)}\right) .
\end{aligned}
$$

The proof of this lemma requires an auxilliary result which we state as a claim. It compares $T_{K, \eta} L_{H}$ with $L_{K \star H}$ (cf. subsection $5.2)$.

Claim. For any nonnegative $t$,

$$
\begin{aligned}
& \left|T_{K, \eta} L_{H} h-L_{K \star H} h\right|(t) \\
& \leqslant \sum_{0 \leqslant s \leqslant m} \frac{\left|h^{(s)}(t)\right|}{s !} \sum_{0 \leqslant j \leqslant s}\left(\begin{array}{l}
s \\
j
\end{array}\right) \mu_{H, j} \int_{\eta t}^{\infty} x^{s-j} \mathrm{~d} K(x) \\
& \quad+\frac{\left|h^{(m)}(t)\right|}{t^{r} m !} \sum_{0 \leqslant j \leqslant m}\left(\begin{array}{c}
m \\
j
\end{array}\right) \mu_{H, j} \int_{0}^{\eta t} \bar{\Delta}_{t, x / t}^{r}\left(h^{(m)}\right) x^{m+r-j} \mathrm{~d} K(x)
\end{aligned}
$$


Proof. By linearity of $T_{K, \eta}$ we have

$$
T_{K, \eta} L_{H}=\sum_{0 \leqslant j \leqslant m} \frac{(-1)^{j}}{j !} \mu_{H, j} T_{K, \eta} \mathrm{D}^{j} .
$$

Applying Theorem 5.4.1 to $h^{(j)}$ in the form of a bound for $\mid T_{K, \eta} h^{(j)}-$ $L_{K, m-j} h^{(j)} \mid$, we obtain

$$
\begin{aligned}
\mid T_{K, \eta} L_{H, m} h- & \sum_{0 \leqslant j \leqslant m} \frac{(-1)^{j}}{j !} \mu_{H, j} L_{K, m-j} h^{(j)} \mid(t) \\
\leqslant \sum_{0 \leqslant j \leqslant m} \frac{\mu_{H, j}}{j !} & \left(\sum_{0 \leqslant l \leqslant m-j} \frac{\left|h^{(j+l)}(t)\right|}{l !} \int_{\eta t}^{\infty} x^{l} \mathrm{~d} K(x)\right. \\
& \left.+\frac{\left|h^{(m)}(t)\right|}{t^{r}(m-j) !} \int_{0}^{\eta t} \bar{\Delta}_{t, x / t}^{r}\left(h^{(m)}\right) x^{m-j+r} \mathrm{~d} K(x)\right) .
\end{aligned}
$$

Using Lemma 2.1.4, the left hand side is the absolute value of $\left(T_{K, \eta} L_{H}-L_{K \star H}\right) h$ evaluated at $t$. Setting $s=l+j$, the right hand side is at most

$$
\begin{aligned}
\sum_{s, j} \mathbb{I}\{j \leqslant s \leqslant m\} \frac{\left|h^{(s)}(t)\right|}{s !}\left(\begin{array}{c}
s \\
j
\end{array}\right) \mu_{H, j} \int_{\eta t}^{\infty} x^{s-j} \mathrm{~d} K(x) \\
+\frac{\left|h^{(m)}(t)\right|}{t^{r} m !}\left(\sum_{0 \leqslant j \leqslant m}\left(\begin{array}{c}
m \\
j
\end{array}\right) \mu_{H, j} \int_{0}^{\eta t} \bar{\Delta}_{t, x / t}^{r}\left(h^{(m)}\right) x^{m-j+r} \mathrm{~d} K(x)\right) .
\end{aligned}
$$

This is our claim.

Proof (of Lemma 5.5.4). First the triangle inequality implies the pointwise inequality

$$
\begin{aligned}
& \left|T_{F_{n}, d_{n}} \mathcal{A} \bar{G}_{n-1}^{(k)}-\sum_{1 \leqslant i \leqslant n-1} L_{G_{n} \natural F_{i}} \bar{F}_{i}^{(k)}\right| \\
& \quad \leqslant \sum_{1 \leqslant i \leqslant n-1}\left|T_{F_{n}, d_{n}} L_{G_{n-1} \natural F_{i}} \bar{F}_{i}^{(k)}-L_{G_{n} \natural F_{i}} \bar{F}_{i}^{(k)}\right| .
\end{aligned}
$$

Since $F_{n} \star\left(G_{n-1} \natural F_{i}\right)=G_{n} \natural F_{i}$, the claim yields for $t$ at least 1,

$$
\begin{aligned}
& \left|T_{F_{n}, d_{n}} L_{G_{n-1} \natural F_{i}} \bar{F}_{i}^{(k)}-L_{G_{n} \natural F_{i}} \bar{F}_{i}^{(k)}\right|(t) \\
& \quad \leqslant \sum_{0 \leqslant s \leqslant m} \frac{\left|\bar{F}_{i}^{(k+s)}(t)\right|}{s !} \sum_{0 \leqslant j \leqslant s}\left(\begin{array}{l}
s \\
j
\end{array}\right) \mu_{G_{n-1} \dashv F_{i}, j} \int_{t d_{n}}^{\infty} x^{s-j} \mathrm{~d} F_{n}(x)
\end{aligned}
$$




$$
\begin{aligned}
+\frac{\left|\bar{F}_{i}^{(k+m)}(t)\right|}{m !} & \sum_{0 \leqslant j \leqslant m}\left(\begin{array}{c}
m \\
j
\end{array}\right) \mu_{G_{n-1} \boxminus F_{i}, j} \\
& \times \int_{0}^{d_{n} t} \bar{\Delta}_{t, x / t}^{r}\left(\bar{F}_{i}^{(k+m)}\right) x^{m+r-j} \mathrm{~d} F_{n}(x) .
\end{aligned}
$$

Since we assume $C_{\rho}$ to be at most 1 , all the $c_{n}$ 's are at most 1 . From Lemma 2.2.3, we deduce that for $t$ at least $t_{1}$,

$$
\left|\bar{F}_{i}^{(k+s)}(t)\right| \leqslant M c_{i}^{\alpha-\epsilon} t^{-k-s} \bar{F}(t) .
$$

Moreover, $\mu_{G_{n-1} \downarrow F_{i}, j} \leqslant \mu_{G, j}$. But $\mu_{G, j}$ is a fixed polynomial in the $C_{l}$ 's and $\mu_{F, l}$ 's, $1 \leqslant l \leqslant j$. Since all these $C_{l}$ 's are at most $C_{\rho}$, we conclude that $\mu_{G_{n-1} \sharp F_{i}, j}$ is at most some fixed constant $M$; this constant does not depend on $n$ and sequences $\left(c_{n}\right)_{n \geqslant 1}$ with $C_{\rho} \leqslant 1$.

Lemma 5.3.3 implies that for any $t$ at least $t_{4}$,

$$
\int_{t d_{n}}^{\infty} x^{s-j} \mathrm{~d} F_{n}(x) \leqslant M d_{n}^{s-j} c_{n}^{\alpha(1-\rho)-\epsilon} t^{s-j} \bar{F}(t) .
$$

It follows that the sum in the first term of (5.5.3) is at most

$$
\begin{aligned}
\sum_{0 \leqslant s \leqslant m} M c_{i}^{\alpha-\epsilon} t^{-k-s} \bar{F}(t) \sum_{0 \leqslant j \leqslant s} d_{n}^{s-j} c_{n}^{\alpha(1-\rho)-\epsilon} t^{s-j} \bar{F}(t) & \\
& \leqslant M t^{-k} \bar{F}(t)^{2} c_{i}^{\alpha-\epsilon} c_{n}^{\alpha(1-\rho)-\epsilon} .
\end{aligned}
$$

Similarly, for the second term in (5.5.3), we use the same bounds and Lemma 2.2.6 to obtain that it is at most

$$
M c_{i}^{\alpha-\epsilon} t^{-k-m} \bar{F}(t) \max _{0 \leqslant j \leqslant m} \int_{0}^{d_{n} t} \bar{\Delta}_{t / c_{i}, x / t}^{r}\left(\bar{F}^{(k+m)}\right) x^{m+r-j} \mathrm{~d} F_{n}(x) .
$$

Using a change of variables, we rewrite the integral terms in this bound as

$$
c_{n}^{m+r-j} \int_{0}^{t d_{n} / c_{n}} \bar{\Delta}_{t / c_{i}, c_{n} x / t}^{r}\left(\bar{F}^{(k+m)}\right) x^{m+r-j} \mathrm{~d} F(x) .
$$

As in the proof of Lemma 5.5.1, we split the integral in this bound as one for $x$ between 0 and $v(t)$, and one between $v(t)$ and $t d_{n} / c_{n}$. We have

$$
\begin{aligned}
\int_{0}^{v(t)} \bar{\Delta}_{t / c_{i}, c_{n} x / t}^{r}\left(\bar{F}^{(k+m)}\right) & x^{m+r-j} \mathrm{~d} F(x) \\
& \leqslant \bar{\Delta}_{t, c_{n} v(t) / t}^{r}\left(\bar{F}^{(k+m)}\right) \mu_{F, m+r-j}
\end{aligned}
$$


and using Lemma 5.3.3 (with $u=v=1$ in it), for $v(t)$ at least $t_{1}$,

$$
\begin{aligned}
& \int_{v(t)}^{t d_{n} / c_{n}} \bar{\Delta}_{t / c_{i}, c_{n} x / t}^{r}\left(\bar{F}^{(k+m)}\right) x^{m+r-j} \mathrm{~d} F(x) \\
& \leqslant M \bar{\Delta}_{t / c_{i}, d_{n}}^{r}\left(\bar{F}^{(k+m)}\right) v(t)^{m+r-j} \bar{F} \circ v(t) .
\end{aligned}
$$

We conclude that for $t$ large enough (5.5.3) is at most

$$
\begin{aligned}
M t^{-k-m} \bar{F}(t) c_{i}^{\alpha-\epsilon}\left(t^{m} \bar{F}(t)\right. & c_{n}^{\alpha(1-\rho)-\epsilon}+c_{n}^{r} \bar{\Delta}_{t, c_{n} v(t) / t}^{r}\left(\bar{F}^{(k+m)}\right) \\
& \left.+c_{n}^{r} \bar{\Delta}_{t, d_{n}}^{r}\left(\bar{F}^{(k+m)}\right) v(t)^{m+r} \bar{F} \circ v(t)\right) .
\end{aligned}
$$

Since $m+r$ is smaller than $\alpha$, the function $s \mapsto s^{m+r} \bar{F}(s)$ is asymptotically equivalent to a nonincreasing function (Bingham, Goldie and Teugels, Theorem 1.5.3). Hence, for $v(t)$ large enough, $t^{m} \bar{F}(t)$ is at most $2 v(t)^{m+r} \bar{F} \circ v(t)$. Therefore, (5.5.3) is at most

$$
\begin{aligned}
M t^{-k-m} \bar{F}(t) c_{i}^{\alpha-\epsilon}\left(\left(c_{n}^{\alpha(1-\rho)-\epsilon}+c_{n}^{r} \bar{\Delta}_{t, d_{n}}^{r}\left(\bar{F}^{(k+m)}\right)\right) v(t)^{m+r} \bar{F} \circ v(t)\right. & \\
& \left.+c_{n}^{r} \bar{\Delta}_{t, c_{n} v(t) / t}^{r}\left(\bar{F}^{(k+m)}\right)\right) .
\end{aligned}
$$

We then use (5.5.2) to finish.

\subsection{Inductions. Define}

$$
\gamma_{m, n}^{(k)}(t)=\left|\bar{G}_{n}^{(k)}-\mathcal{A}_{m} \bar{G}_{n}^{(k)}\right|_{k+m, t}
$$

Our proof of Theorem 2.3.1 consists in bounding $\gamma_{m, n}^{(k)}(t)$ uniformly in $n$. This is done by induction on $n$, and we first need to settle the case $n=2$.

Lemma 5.6.1. Take $\rho$ less than $\alpha /(\alpha+k)$. There exists a function $\eta_{0}$ with limit 0 at infinity, such that whenever $d_{1}$ and $d_{2}$ are at most $1 / 2$ and $k+m<\omega$,

$$
\gamma_{m, 2}^{(k)}(t) \leqslant \eta_{0}(t)
$$

Proof. Proposition 5.1.1 shows that

$$
\bar{G}_{2}=T_{F_{1}, 1-d_{2}} \bar{F}_{2}+T_{F_{2}, d_{2}} \bar{F}_{1}+M_{1 /\left(1-d_{2}\right)} \bar{F}_{1} M_{1 / d_{2}} \bar{F}_{2}
$$


while for $k$ at least 1 ,

$$
\begin{aligned}
\bar{G}_{2}^{(k)}=T_{F_{1}, 1-d_{2}} & \bar{F}_{2}^{(k)}+T_{F_{2}, d_{2}} \bar{F}_{1}^{(k)} \\
& -\sum_{1 \leqslant j \leqslant k-1} M_{1 /\left(1-d_{2}\right)} \bar{F}_{1}^{(j)} M_{1 / d_{2}} \bar{F}_{2}^{(k-j)} .
\end{aligned}
$$

Let $r$ be a nonnegative number less than 1 and $\omega-k-m$. We use a slight modification of Lemma 5.5.1 with $n$ equal 2 to obtain

$$
\begin{aligned}
& \left|\left(T_{F_{1}, 1-d_{2}}-L_{F_{1}, m}\right) \bar{F}_{2}^{(k)}\right|_{k+m, t} \\
& \quad \leqslant M c_{2}^{\alpha-\rho(\alpha+k+m)-\epsilon}\left(\bar{\Delta}_{t, v(t) / t}^{r}\left(\bar{F}^{(k+m)}\right)+v(t)^{m+r} \bar{F} \circ v(t)\right) .
\end{aligned}
$$

Permuting $F_{1}$ and $F_{2}$ yields an upper bound for the error committed in approximating $T_{F_{2}, d_{2}} \bar{F}_{1}^{(k)}$ by $L_{F_{2}, m} \bar{F}_{1}^{(k)}$.

Combined with (5.6.2), this proves the lemma when $k$ is 1 .

When $k$ vanishes, since $m$ is smaller than $\alpha$,

$$
M_{1 / d_{2}} \bar{F}_{2}(t) M_{1 /\left(1-d_{2}\right)} \bar{F}_{1}(t)=o\left(t^{-m} \bar{F}(t)\right)
$$

as $t$ tends to infinity. Since $M_{1 / d_{2}} \bar{F}_{2}=M_{c_{2}^{1-\rho}} \bar{F}$ this is uniform in $d_{1}$ and $d_{2}$ at most $1 / 2$. Combined with (5.6.1), this proves the result for $k$ vanishing.

When $k$ is larger than 1 , let $\epsilon$ be less than $\alpha(1-\rho)-k \rho$. We use Lemma 2.2.3 to obtain

$$
\begin{aligned}
& \left|M_{1 / d_{2}} \bar{F}_{2}^{(i)} M_{1 /\left(1-d_{2}\right)} \bar{F}_{1}^{(k-i)}\right|(t) \\
& \quad=\left|c_{2}^{-i} \bar{F}^{(i)}\left(t d_{2} / c_{2}\right) c_{1}^{i-k} \bar{F}^{(k-i)}\left(t\left(1-d_{2}\right) / c_{1}\right)\right| \\
& \leqslant M t^{-i} c_{2}^{\alpha(1-\rho)-i \rho-\epsilon} \bar{F}(t) t^{-k+i} c_{1}^{\alpha-\epsilon} \bar{F}(t)
\end{aligned}
$$

which is at most $M t^{-k} \bar{F}(t)^{2}$. We conclude as before.

The next step is to show that $\gamma_{m, n}^{(k)}$ can be bounded by induction. For this purpose, we need another bound that will control the sum involving the multiplication operators in (5.2.1).

Lemma 5.6.2. Let $k$ be at least 2. Let $\epsilon$ be a positive real number. Assume that all $d_{i}$ 's are smaller than $1 / 2$. There exist $t_{9}$ and $M$ such that for any $t$ at least $t_{9}$, any positive integers $j, k$ with $j \leqslant k-1$,

$$
\begin{aligned}
& \sum_{i \geqslant 2}\left|M_{1 / d_{i}} \bar{F}_{i}^{(j)} M_{1 /\left(1-d_{i}\right)} \bar{G}_{i-1}^{(k-j)}\right|_{k+m, t} \\
& \leqslant M t^{m} \bar{F}(t)\left(C_{\alpha-\epsilon /(1-\rho)}+\sup _{i \geqslant 2} \gamma_{0, i}^{(k-j)}(t / 2)\right) C_{\alpha-\rho(\alpha+j)-\epsilon} .
\end{aligned}
$$


Proof. Define $\delta=\epsilon /(1-\rho)$. Under our assumptions, all the $c_{i}$ 's are at most 1. Consequently, $d_{i} / c_{i}$ is at least 1 and Lemma 2.2.3 (taking $c$ to be 1 in that lemma) implies that for $s$ at least $t_{1}$,

$$
\left|M_{1 / d_{i}} \bar{F}_{i}^{(j)}(s)\right|=c_{i}^{-j}\left|\bar{F}^{(j)}\left(s d_{i} / c_{i}\right)\right| \leqslant c_{i}^{-j} M\left(s d_{i} / c_{i}\right)^{-j} \bar{F}\left(s d_{i} / c_{i}\right) .
$$

Applying Lemma 2.2.4, using again the fact that $d_{i} / c_{i}$ is at least 1 , we see that for $s$ at least $t_{1}$ and $t_{2}$,

$$
\left|M_{1 / d_{i}} \bar{F}_{i}^{(j)}(s)\right| \leqslant M c_{i}^{-j+(j+\alpha-\delta)(1-\rho)} s^{-j} \bar{F}(s) .
$$

Since $\mathcal{A}_{0} \bar{G}_{i}^{(k-j)}=\sum_{1 \leqslant l \leqslant i} \bar{F}_{l}^{(k-j)}$, the triangle inequality yields

$$
\left|\bar{G}_{i}^{(k-j)}(s)\right| \leqslant \sum_{1 \leqslant l \leqslant i}\left|\bar{F}_{l}^{(k-j)}(s)\right|+\gamma_{0, i}^{(k-j)}(s) s^{-k+j} \bar{F}(s) .
$$

Using again Lemma 2.2.3 and the fact that the $c_{i}$ 's are at most 1, we see that for $s$ at least $t_{1}$,

$$
\begin{aligned}
\left|\bar{G}_{i}^{(k-j)}(s)\right| & \leqslant M \sum_{1 \leqslant l \leqslant i} c_{l}^{\alpha-\delta} s^{-k+j} \bar{F}(s)+\gamma_{0, i}^{(k-j)}(s) s^{-k+j} \bar{F}(s) \\
& \leqslant M s^{-k+j} \bar{F}(s)\left(C_{\alpha-\delta}+\sup _{n \geqslant 2} \gamma_{0, n}^{(k-j)}(s)\right) .
\end{aligned}
$$

Consequently, since $d_{i}$ is at most $1 / 2$, we obtain that for $t$ large enough,

$$
\begin{aligned}
& \sum_{i \geqslant 2} \sup _{s \geqslant t} s^{k+m}\left|M_{1 / d_{i}} \bar{F}_{i}^{(j)}(s) M_{1 /\left(1-d_{i}\right)} \bar{G}_{i-1}^{(k-j)}(s)\right| / \bar{F}(s) \\
& \leqslant M \sup _{s \geqslant t} s^{m} \bar{F}(s / 2)\left(C_{\alpha-\delta}+\sup _{n \geqslant 2} \gamma_{0, n}^{(k-j)}(s / 2)\right) C_{(j+\alpha-\delta)(1-\rho)-j},
\end{aligned}
$$

the factor $1 / 2$ in $s / 2$ coming from the multiplication operator acting on $\bar{G}_{i-1}^{(k-1)}$ and our assumption that $d_{i}$ is at most $1 / 2$. One more application of Potter's bound allows us to replace $\bar{F}(s / 2)$ by $\bar{F}(s)$ in the upper bound. Since $\gamma_{0, i}^{(k-j)}(\cdot)$ is nonincreasing and $s \mapsto s^{m} \bar{F}(s)$ is asymptotically equivalent to an ultimately nonincreasing function (Bingham, Goldie and Teugels, 1989, Theorem 1.5.3), we can remove the supremum in $s$ in the above bound (and, of course, we increase $M$ in doing that). 
Our next lemma shows that $\gamma_{m, n}^{(k)}(\cdot)$ can be bounded by induction on $k$, whenever $k$ is at least 2 . We define

$$
\eta_{\sigma}(t)=(v(t) / t)^{\gamma}+v(t)^{\sigma} \bar{F} \circ v(t) .
$$

Lemma 5.6.3. Let $\epsilon$ be a positive real number, and let $k$ be a positive integer. There exist positive numbers $M$ and $t_{10}$ such that for any integer $n$ at least 2 and any sequence $\left(c_{n}\right)_{n \geqslant 1}$ with $C_{\gamma \rho} \vee C_{\alpha-(\alpha+k+m) \rho-\epsilon} \leqslant 1$ and $d_{n} \leqslant 1 / 2$,

$$
\begin{aligned}
\gamma_{m, n}^{(k)}(t) \leqslant & M\left(1+\max _{1 \leqslant j \leqslant k-1} \sup _{i \geqslant 2} \gamma_{0, i}^{(k-j)}(t / M)\right) \eta_{m}(t / M) \\
& +M \gamma_{m, 2}^{(k)}(t / M) .
\end{aligned}
$$

In the statement of this lemma, we agree that $\max _{1 \leqslant j \leqslant 0}$ is 0 .

Proof. For $u$ positive, the inequality $\bar{\Delta}_{t, u}^{r}(h) \leqslant u^{\gamma-r} \bar{\Delta}_{t, u}^{\gamma}(h)$ holds. We will take $r$ to be 0 in this inequality and apply it in the bounds of the previous subsection. Under the assumption of Theorem 2.3.1, we also have $\lim \sup _{t \rightarrow \infty} \bar{\Delta}_{t, 1 / 2}^{0}\left(\bar{F}^{(k+m)}\right)$ finite. Using (5.2.1), Lemma 5.5.1, Corollary 5.5.3 and 5.5.4, we obtain that there exists $M$ and $t_{10}^{\prime}$ such that for any $t$ at least $t_{10}^{\prime}$ and any $n$ at least 3,

$$
\begin{aligned}
\gamma_{m, n}^{(k)}(t) \leqslant & M c_{n}^{\alpha-(\alpha+k+m) \rho-\epsilon}\left((v(t) / t)^{\gamma}+v(t)^{m} \bar{F} \circ v(t)\right) \\
& +\gamma_{m, n-1}^{(k)}\left(t\left(1-d_{n}\right)\right)\left(1+M d_{n}\right) \\
& +M\left(c_{n}^{\alpha(1-\rho)-\epsilon}+d_{n}^{\gamma}\right) v(t)^{m} \bar{F} \circ v(t)+M c_{n}^{\gamma}(v(t) / t)^{\gamma} \\
& +\sum_{1 \leqslant j \leqslant k-1}\left|M_{1 / d_{n}} \bar{F}_{n}^{(j)} M_{1 /\left(1-d_{n}\right)} \bar{G}_{n-1}^{(k-j)}\right|_{k+m, t} .
\end{aligned}
$$

Collecting the terms and using that all the $c_{n}$ 's are at most 1 ,

$$
\begin{aligned}
\gamma_{m, n}^{(k)}(t) \leqslant & M\left(c_{n}^{\alpha-(\alpha+k+m) \rho-\epsilon}+c_{n}^{\gamma \rho}\right) \eta_{m}(t) \\
& +\gamma_{m, n-1}^{(k)}\left(t\left(1-d_{n}\right)\right)\left(1+M d_{n}\right) \\
& +\sum_{1 \leqslant j \leqslant k-1}\left|M_{1 / d_{n}} \bar{F}_{n}^{(j)} M_{1 /\left(1-d_{n}\right)} \bar{G}_{n-1}^{(k-j)}\right|_{k+m, t}
\end{aligned}
$$

Taking $t_{10}^{\prime}$ large enough, we can assume that $\eta_{m}(\cdot)$ is nonincreasing on $\left[t_{10}^{\prime}, \infty\right)$. Dropping the subscript $m$ and superscript $k$ temporarily, inequality (5.6.3) has the form

$$
\gamma_{n}(t) \leqslant a_{n}(t)+\gamma_{n-1}\left(t\left(1-d_{n}\right)\right)\left(1+M d_{n}\right),
$$


where $a_{n}(\cdot)$ is the nonnegative and nonincreasing function on $\left[t_{10}^{\prime}, \infty\right)$ given by

$$
\begin{aligned}
& a_{n}(t)=M\left(c_{n}^{\alpha-(\alpha+k+m) \rho-\epsilon}+c_{n}^{\rho \gamma}\right) \eta_{m}(t) \\
& \quad+\sum_{1 \leqslant j \leqslant k-1}\left|M_{1 / d_{n}} \bar{F}_{n}^{(j)} M_{1 /\left(1-d_{n}\right)} \bar{G}_{n-1}^{(k-j)}\right|_{k+m, t} .
\end{aligned}
$$

For $i+1$ at most $n$, define $A_{i, n}=\prod_{i+1 \leqslant j \leqslant n}\left(1-d_{j}\right)$ and $B_{i, n}=\prod_{i+1 \leqslant j \leqslant n}\left(1+M d_{j}\right)$. We also set $A_{n, n} \stackrel{=}{=} B_{n, n}=1$. By induction, inequality (5.6.3) implies

$$
\gamma_{n}(t) \leqslant \sum_{3 \leqslant i \leqslant n} B_{i, n} a_{i}\left(t A_{i, n}\right)+B_{2, n} \gamma_{2}\left(t A_{2, n}\right) .
$$

Set $A=\prod_{j \geqslant 1}\left(1-d_{j}\right)$ and $B=\prod_{j \geqslant 1}\left(1+M d_{j}\right)$. Since all the $a_{n}(\cdot)$ 's are nonnegative and nonincreasing, we have

$$
\gamma_{n}(t) \leqslant \sum_{i \geqslant 3} B a_{i}(t A)+B \gamma_{2}(t A)
$$

The inequality $\log (1-x) \geqslant-2 x$ for $x$ nonnegative and at most $1 / 2$ implies

$$
A \geqslant \exp \left(-2 \sum_{j \geqslant 1} d_{j}\right)=\exp \left(-2 C_{\rho}\right)
$$

while the the inequality $\log (1+x) \leqslant x$ implies

$$
B \leqslant \exp \left(\sum_{j \geqslant 1} M d_{j}\right)=\exp \left(M C_{\rho}\right)
$$

Consequently,

$$
\gamma_{n}(t) \leqslant e^{M C_{\rho}}\left(\sum_{i \geqslant 3} a_{i}\left(t e^{-2 C_{\rho}}\right)+\gamma_{2}\left(t e^{-2 C_{\rho}}\right)\right) .
$$

We also have

$$
\begin{aligned}
\sum_{i \geqslant 3} a_{i}(s) \leqslant & M\left(C_{\alpha-(\alpha+k+m) \rho-\epsilon}+C_{\rho \gamma}\right) \eta_{m}(s) \\
& +\sum_{1 \leqslant j \leqslant k-1} \sum_{i \geqslant 3}\left|M_{1 / d_{i}} \bar{F}_{i}^{(j)} M_{1 /\left(1-d_{i}\right)} \bar{G}_{i-1}^{(j-k)}\right|_{k+m, s} .
\end{aligned}
$$


Applying Lemma 5.6.2, this bound is at most

$$
\begin{aligned}
& M\left(C_{\alpha-(\alpha+k+m) \rho-\epsilon}+C_{\rho \gamma}\right) \eta_{m}(s) \\
& \quad+M s^{m} \bar{F}(s)\left(C_{\alpha-\epsilon /(1-\rho)}+\max _{1 \leqslant j \leqslant k-1} \sup _{i \geqslant 2} \gamma_{0, i}^{(k-j)}(s / 2)\right) C_{\alpha-\rho(\alpha+k)-\epsilon} .
\end{aligned}
$$

Since $s^{m} \bar{F}(s)$ is at most $\eta_{m}(s)$ for $s$ at least 1 , this bound yields

$$
\begin{gathered}
\gamma_{n}(t) \leqslant M\left(C_{\alpha-(\alpha+k+m) \rho-\epsilon}+C_{\gamma \rho}+1+\max _{1 \leqslant j \leqslant k-1} \sup _{i \geqslant 2} \gamma_{0, i}^{(k-j)}\left(t e^{-2 C_{\rho}} / 2\right)\right) \\
\times \eta_{m}\left(t e^{-2 C_{\rho}}\right)+e^{M C_{\rho}} \gamma_{2}\left(t e^{-2 C_{\rho}}\right) .
\end{gathered}
$$

To conclude the proof, if $C_{\rho \gamma}$ is at most 1 , so are all the $c_{i}$ 's and $C_{\rho}$ is at most $C_{\rho \gamma}$.

We need an analogue of Lemma 5.6.3 when $k$ vanishes. For this purpose, we state the analogue of Lemma 5.6.2 in this case.

Lemma 5.6.4. Assume that $C_{\rho}$ is at most 1 and that all $d_{i}$ 's are at most $1 / 2$. For $t$ larger than some $t_{11}$, the following inequality holds:

$$
\sum_{i \geqslant 1}\left|M_{1 / d_{i}} \bar{F}_{i} M_{1 /\left(1-d_{i}\right)} \bar{G}_{i-1}\right|_{k+m, t} \leqslant C_{\alpha(1-\rho)-\epsilon}^{2} t^{m} \bar{F}(t) .
$$

Proof. Set $\delta=\epsilon /(1-\rho)$. Apply Lemma 2.2.4 to obtain that for $s$ at least $t_{2}$,

$$
M_{1 / d_{i}} \bar{F}_{i}(s)=\bar{F}\left(s d_{i} / c_{i}\right) \leqslant c_{i}^{(1-\rho)(\alpha-\delta)} \bar{F}(s) .
$$

Since $d_{i}$ is at most 1/2, Lemma 5.3.1 implies

$$
M_{1 /\left(1-\mathrm{d}_{i}\right)} \bar{G}_{i-1}(s) \leqslant \sum_{n \geqslant 1} \bar{F}\left(s / 2 c_{n}^{1-\rho}\right),
$$

which, by Lemma 2.2.4 is at most $M \sum_{n \geqslant 1} c_{n}^{(1-\rho)(\alpha-\delta)} \bar{F}(s)$ for $s$ large enough. This implies the conclusion.

The next lemma is Theorem 2.3.1 stated in an other way. 
Lemma 5.6.5. Let $\epsilon$ be a positive number at most 1 . There exists a function $\eta(\cdot)$ with limit 0 at infinity, such that, for any $k$ and $m$ with $k+m$ smaller than $\omega$ and $m$ smaller than $\alpha$, any $n$ at least 2 , and any $t$ at least 1 say, any nonnegative sequence $\left(c_{i}\right)_{i \geqslant 1}$ with $\sup _{i \geqslant 1} d_{i} \leqslant 1 / 2$ and both $C_{\gamma \rho}$ and $C_{\alpha-(\alpha+k+m) \rho-\epsilon}$ at most 1 ,

$$
\gamma_{m, n}^{(k)}(t) \leqslant \eta(t)
$$

Proof. For $k=1$, Lemma 5.6.3 implies

$$
\gamma_{m, n}^{(1)}(t) \leqslant M \eta_{m}(t / M)+M \gamma_{m, 2}^{(1)}(t / M)
$$

The result follows then from the assumptions and Lemma 5.6.1.

By induction on $k$, Lemmas 5.6.3 and 5.6.2 imply the result for $k$ at least 1.

When $k$ vanishes, Lemma 5.6.4 and the same arguments as those in the proof of Lemma 5.6.3 show that

$\gamma_{m, n}^{(0)}(t) \leqslant M\left(C_{(1-\rho)(\alpha-\epsilon)}+1\right) \eta_{m}(t / M)+M \gamma_{m, 2}^{(0)}+M C_{\alpha(1-\rho)-\epsilon}^{2} t^{m} \bar{F}(t)$

(compare with (5.6.3)). The result follows.

5.7. Conclusion. To obtain Theorem 2.3.1, it mostly remains to show that as $n$ tends to infinity, $\gamma_{m, n}(t)$ converges to $\bar{G}^{(k)}-$ $\left.\mathcal{A}_{m} \bar{G}^{(k)}\right|_{k+m, t}$. This is achieved in two steps, one consisting in proving that the sequence of approximations converges, the other one in proving that the sequence of functions $G_{n}^{(k)}$ converges to $G^{(k)}$.

Lemma 5.7.1. Assume that $C_{1-\rho}$ and $|c|_{\infty}$ are at most 1 . Then there exists $t_{12}$ and $M$ such that for any $t$ at least $t_{12}$,

$$
\left|\mathcal{A}_{m} \bar{G}_{n}^{(k)}-\mathcal{A}_{m} \bar{G}^{(k)}\right|_{k, t} \leqslant M \sum_{i \geqslant n+1} c_{i}^{1-\rho}
$$

Remark. For our purpose, it is enough that $\mathcal{A}_{m} \bar{G}_{n}^{(k)}$ converges pointwise to $\mathcal{A}_{m} \bar{G}^{(k)}$. In order to simulate properly tail behavior, it would be desirable to have convergence in $|\cdot|_{k+m, t}$ norm. 
Proof. From the definition of the approximation and since both $\mu_{G_{n} \sharp F_{i}, 0}$ and $\mu_{G \natural F_{i}, 0}$ equal 1 for $i$ at most $n$,

$$
\begin{aligned}
\mathcal{A}_{m} \bar{G}_{n}^{(k)}-\mathcal{A}_{m} \bar{G}^{(k)}= & \sum_{0 \leqslant j \leqslant m} \frac{(-1)^{j}}{j !} \sum_{1 \leqslant i \leqslant n}\left(\mu_{G_{n} \natural F_{i}, j}-\mu_{G \natural F_{i}, j}\right) \mathrm{D}^{j+k} \bar{F}_{i} \\
& -\sum_{0 \leqslant j \leqslant m} \frac{(-1)^{j}}{j !} \sum_{i \geqslant n+1} \mu_{G \natural F_{i}, j} \mathrm{D}^{j+k} \bar{F}_{i} \cdot(5.7 .1)
\end{aligned}
$$

Write $Y=\sum_{\substack{1 \leqslant j \leqslant n \\ j \neq i}} c_{j} X_{j}$ and $R=\sum_{\substack{j \geqslant n+1 \\ j \neq i}} c_{j} X_{j}$. Then, for $j$ at least 1 ,

$$
\mu_{G \natural F_{i}, j}-\mu_{G_{n} \natural F_{i}, j}=E\left((Y+R)^{j}-Y^{j}\right)=\sum_{1 \leqslant l \leqslant j}\left(\begin{array}{l}
j \\
l
\end{array}\right) E R^{l} E Y^{j-l} .
$$

Applying Lemma 5.3.5, we see that for any $l$ at least 1,

$$
E R^{l} \leqslant \sum_{i \geqslant n+1} c_{i}^{l(1-\rho)} \mu_{F, l} \leqslant \sum_{i \geqslant n+1} c_{i}^{1-\rho} \mu_{F, l},
$$

while for $l$ less than $j$, by the same token and under the assumption of the lemma,

$$
E Y^{j-l} \leqslant \mu_{G, j-l} \leqslant C_{(j-l)(1-\rho)} \mu_{F, j-l} \leqslant \mu_{F, j-l} .
$$

Consequently, for $j$ at least 1 ,

$$
\begin{aligned}
0 \leqslant \mu_{G \natural F_{i}, j}-\mu_{G_{n} \natural F_{i}, j} & \leqslant \sum_{1 \leqslant l \leqslant j}\left(\begin{array}{l}
j \\
l
\end{array}\right) \mu_{F, l} \mu_{F, j-l} \sum_{i \geqslant n+1} c_{i}^{1-\rho} \\
& \leqslant \mu_{F \star F, j} \sum_{i \geqslant n+1} c_{i}^{1-\rho} .
\end{aligned}
$$

For $t$ at least $t_{1}$, Lemma 2.2.3 implies

$$
\left|\mathrm{D}^{j+k} \bar{F}_{i}(t)\right| \leqslant M c_{i}^{\alpha-\epsilon} t^{-k-j} \bar{F}(t) .
$$

So, the first double sum in (5.7.1) is at most

$$
M t^{-k} \bar{F}(t) \sum_{i \geqslant n+1} c_{i}^{1-\rho}
$$


To bound the second double sum, we use Lemma 5.3.5 to obtain, for $j$ at least 1 .

$$
\mu_{G \sharp F_{i}, j} \leqslant \mu_{G, j} \leqslant C_{1-\rho} \mu_{F, j} .
$$

Hence, the second double sum in (5.7.1) is at most

$$
M \sum_{i \geqslant n+1} c_{i}^{\alpha-\epsilon} t^{-k} \bar{F}(t)
$$

This concludes the proof.

The next lemma is the only place where the boundedness and continuity of $F^{(k)}$ is used. Its proof is an adaptation of that of Proposition 9.1.6 in Dudley (1989).

Lemma 5.7.2. Assume that $F$ is $k$-times continuously differentiable on $(0, \infty)$, and that $F^{(k)}$ is bounded and in $L^{1}(\mathrm{~d} x)$. Then, $\lim _{n \rightarrow \infty} G_{n}^{(k)}=G^{(k)}$ pointwise.

\section{Proof. Write}

$$
G_{n}(t)=\int_{0}^{t} F_{1}(t-y) \mathrm{d} G_{n} \curvearrowleft F_{1}(y) .
$$

Since $F^{(k)}$ exists and is in $L^{1}(\mathrm{~d} x)$, so is $F_{1}$. Then

$$
G_{n}^{(k)}(t)=\int_{0}^{t} F_{1}^{(k)}(t-y) \mathrm{d} G_{n} \llbracket F_{1}(y) .
$$

But the sequence $\left(G_{n} \natural F_{1}\right)_{n \geqslant 1}$ converges weakly* to the continuous distribution function $G \natural F_{1}$ and since $F_{1}^{(k)}$ is continuous and bounded, $G_{n}^{(k)}(t)$ converges to

$$
\int_{0}^{t} F_{1}^{(k)}(t-y) \mathrm{d} G \natural F_{1}(y)=\left(F_{1} \star\left(G \bigsqcup F_{1}\right)\right)^{(k)}(t)=G^{(k)}(t) .
$$

To obtain Theorem 2.3.1 when the $c_{i}$ 's are nonnegative, it remains to do some rewriting of Lemma 5.6.5. For this purpose, define the sets of nonnegative sequences

$$
\mathcal{C}_{\alpha, \omega, \gamma}=\left\{c \in[0, \infty)^{\mathbb{N}^{*}}: N_{\alpha, \gamma, \omega}(c) \leqslant 1\right\} .
$$


The following is exactly Theorem 2.3.1 but for the positive case and everywhere smooth distribution function.

Proposition 5.7.3. Let $\bar{F}$ in $S R_{-\alpha, \omega}$ and let $k, m$ be two nonnegative integers with $k+m$ smaller than $\omega$ and $m$ smaller than $\alpha$. Let $\gamma$ be a positive real number smaller than both 1 and $\omega-k-m$. Assume that $F^{(k)}$ is countinuous and bounded. There exists a function $\eta(\cdot)$ which tends to 0 at infinity and a real number $t_{13}$ such that for any $t$ at least $t_{13}$ and any sequence $c$ in $\mathcal{C}_{\alpha, \omega, \gamma}$

$$
\left|\bar{G}_{c}^{(k)}-\mathcal{A}_{m} \bar{G}_{c}^{(k)}\right|_{k+m, t} \leqslant \eta(t) .
$$

Proof. For $\epsilon$ positive, define $\rho=(1 / 2) \wedge(\alpha /(\alpha+\omega))$. Let $c$ be a sequence in $\mathcal{C}_{\alpha, \omega, \gamma}$. By definition of $\mathcal{C}_{\alpha, \omega, \gamma}$ the series $C_{\rho \gamma}$ is at most 1. Moreover, by taking $\epsilon$ small enough, $C_{\alpha-\epsilon}$ is at most $C_{\rho \gamma}$, hence at most 1 .

Since $k+m+\gamma<\omega$, we also have $\rho<\alpha /(\alpha+\gamma+k+m)$, that is $\alpha-\rho(\alpha+k+m)>\rho \gamma$. Hence, for $\epsilon$ small enough $C_{\alpha-(\alpha+k+m) \rho-\epsilon}$ is at most 1 .

To conclude the proof, lemmas 5.7.1 and 5.7.2 show that

$$
\bar{G}^{(k)}-\mathcal{A}_{m} \bar{G}^{(k)}=\lim _{n \rightarrow \infty} \bar{G}_{n}^{(k)}-\mathcal{A}_{m} \bar{G}_{n}^{(k)}
$$

pointwise. But for any $n$ at least 2 and any $t$ at least $t_{1}$, Lemma 5.6.5 yields

$$
\left|\bar{G}_{n}^{(k)}(t)-\mathcal{A}_{m} \bar{G}_{n}^{(k)}(t)\right| \leqslant \eta(t) t^{-m-k} \bar{F}(t)
$$

Hence the same inequality holds with $\bar{G}^{(k)}$.

\section{Removing the sign restriction on the random variables.}

In section 5, we assumed that the random variables are positive. Our goal in this section is to remove this assumption. But we will keep the assumption that $F^{(k)}$ and $M_{-1} F^{(k)}$ exist, are bounded and continuous on the whole positive half line. The basic argument consists in conditioning. To be specific, define the random set of all indices corresponding to a nonpositive random variable, that is

$$
I=\left\{i \in \mathbb{N}^{*}: X_{i} \leqslant 0\right\}
$$


Write $H_{I}$ for the distribution function of $\sum_{i \in I} c_{i} X_{i}$ and $G_{I}$ for that of $\sum_{i \in \mathbb{N}^{*} \backslash I} c_{i} X_{i}$. Let $F_{+}$(resp. $F_{-}$) be the distribution function of $X_{1}$ say, given that $X_{1}$ is positive (resp. nonpositive), that is

$$
\overline{F_{+}}=\bar{F} / \bar{F}(0) \quad \text { on } \quad(0, \infty)
$$

and

$$
F_{-}=F / F(0) \quad \text { on } \quad(-\infty, 0]
$$

We write $F_{+, i}\left(\operatorname{resp} . F_{-, i}\right)$ for $M_{c_{i}} F_{+}$(resp. $\left.M_{c_{i}} F_{-}\right)$. Note that there is no ambiguity in this notation for when $c$ is nonnegative, $\left(M_{c} F\right)_{+}=M_{c}\left(F_{+}\right)$and $\left(M_{c} F\right)_{-}=M_{c}\left(F_{-}\right)$. Given $I$, the results of section 5 can be applied to both $M_{-1} H_{I}$ and $G_{I}$, using respectively the distribution functions $M_{-1} F_{-}$and $F_{+}$instead of $F$. The distribution function of the whole series $\langle c, X\rangle$ is $E H_{I} \star G_{I}$. The identity

$$
\overline{H_{I} \star G_{I}}(t)=\int_{-\infty}^{0} \bar{G}_{I}(t-x) \mathrm{d} H_{I}(x),
$$

suggests the relevance to the operator

$$
U_{H} h(t)=\int_{-\infty}^{0} h(t-x) \mathrm{d} H(x)
$$

in term of which $\overline{H_{I} \star G_{I}}=U_{H_{I}} \bar{G}_{I}$. We then need to prove the analogue of some of the results of section 5 on $T_{F, \eta}$, but now for the operator $U_{H}$. The first one which we will prove implies $\mathrm{D}^{k} U_{H} \bar{G}=U_{H} \bar{G}^{(k)}$. It is then clear how the proof of the asymptotic expansion for the distribution function $K$ of $\langle c, X\rangle$ and its derivatives will go. Indeed, we will have

$$
K^{(k)}=E U_{H_{I}} \bar{G}_{I}^{(k)}=E U_{H_{I}} \mathcal{A}_{m} \bar{G}_{I}^{(k)}+E U_{H_{I}}\left(\bar{G}_{I}^{(k)}-\mathcal{A}_{m} \bar{G}_{I}^{(k)}\right) .
$$

We will see that $U_{H}$ is a contraction for the right norm. Hence, equality (6.0.1) combined with the results of section 5 gives the asymptotic expansion

$$
K^{(k)} \sim E U_{H_{I}} \mathcal{A}_{m} \bar{G}_{I}^{(k)}
$$

We will be able to approximate $U_{H_{I}}$ by $L_{H_{I}}$. Because the error term is bounded uniformly with respect to the sequence $\left(c_{i}\right)_{i \geqslant 1}$, we will be able to permute expectation and asymptotic expansions. 
6.1. Elementary properties of $\boldsymbol{U}_{\boldsymbol{H}}$. Since we are interested in expansions of derivatives, our first elementary result deals with the composition of the derivative and $U_{H}$. It asserts that those two operators commute whenever acting on sufficiently regular functions.

Lemma 6.1.1. Let $h$ be a function on the nonnegative half line with Lebesgue integrable $k$-th derivative, and such that for any nonnegative $l$ at most $k$,

$$
\lim _{t \rightarrow \infty} h^{(l)}(t)=0 .
$$

Then, $\mathrm{D}^{k} U_{H} h=U_{H} \mathrm{D}^{k} h$ almost everywhere.

Proof. By induction it suffices to prove the result for $k$ equal 1 . We write

$$
U_{H} h(t)=-\iint h^{\prime}(y-x) \mathbb{I}\{y>t\} \mathrm{d} y \mathrm{~d} H(x) .
$$

The function $U_{H} h^{\prime}$ is Lebesgue integrable for

$$
\int\left|\int h^{\prime}(y-x) \mathrm{d} H(x)\right| \mathrm{d} y \leqslant \iint\left|h^{\prime}(y-x)\right| \mathrm{d} y \mathrm{~d} H(x)=\left|h^{\prime}\right|_{L^{1}(\mathrm{~d} x)} .
$$

Applying Fubini's theorem,

$$
U_{H} h(t)=-\int_{t}^{\infty} \int_{-\infty}^{0} h^{\prime}(y-x) \mathrm{d} H(x) \mathrm{d} y=-\int_{t}^{\infty} U_{H} h^{\prime}(y) \mathrm{d} y .
$$

Since we proved that $U_{H} h^{\prime}$ is Lebesgue integrable, it is almost everywhere the derivative of $U_{H} h$.

In particular, if $F^{(k)}$ is continuous and bounded and $i$ is not in $I$, then the analogue to the expression obtained in the proof of Lemma 5.7 .2 , is

$$
\bar{G}_{I}^{(k)}=\int_{0}^{t} \bar{F}_{+, i}^{(k)}(t-y) \mathrm{d} G_{I} \natural F_{+, i}(y) .
$$

Thus, $\bar{G}_{I}^{(k)}$ is bounded and integrable with respect to the Lebesgue measure. Moreover, since $\bar{F}$ is smoothly varying with negative exponent $-\alpha$, its $k$-th derivative tends to 0 at infinity. Then (6.1.1) shows that $\bar{G}_{I}^{(l)}$ tends to 0 at infinity for any nonnegative $l$ at most $k$. Consequently,

$$
{\overline{H_{I} \star G_{I}}}^{(k)}=U_{H_{I}} \bar{G}_{I}^{(k)} .
$$


Our next result allows us to replace $\bar{G}_{I}^{(k)}$ by its asymptotic expansion when looking for an expansion for $U_{H_{I}} \bar{G}_{I}^{(k)}$.

Lemma 6.1.2. For any nonnegative $t$, the operator $U_{H}$ is a contraction with respect to the norms $|\cdot|_{p, t}$.

Proof. Let $h$ be a function whose $|\cdot|_{p, t}$-norm is finite. Since $t \mapsto t^{p} / \bar{F}(t)$ is nondecreasing on the nonnegative half line

$$
\begin{aligned}
\left|\frac{t^{p}}{\bar{F}(t)} U_{H} h(t)\right| & \leqslant \int_{-\infty}^{0} \frac{t^{p}}{\bar{F}(t)}|h(t-x)| \mathrm{d} H(x) \\
& \leqslant \int_{-\infty}^{0} \frac{(t-x)^{p}}{\bar{F}(t-x)}|h(t-x)| \mathrm{d} H(x) .
\end{aligned}
$$

The integrand is at most $|h|_{p, t}$; so is the integral, for $H$ is a distribution function.

6.2. Basic expansion of $\boldsymbol{U}_{\boldsymbol{H}}$. We now show that $U_{H}$ has an expansion $L_{H, m}$, and therefore behaves similarly to $T_{F, \eta}$. Indeed, the next lemma should be compared to Theorem 5.4.1.

Lemma 6.2.1. Let $m$ be a positive integer and let $r$ be in $[0,1)$. Let $H$ be a distribution funciton on the nonpositive half line with finite $m$-th moment. If $h$ is smoothly varying of order $m+r$, then

$$
\begin{aligned}
\left|\left(U_{H}-L_{H, m}\right) h\right|(t) \leqslant & \sum_{0 \leqslant j \leqslant m} \frac{\left|h^{(j)}(t)\right|}{j !} \int_{-\infty}^{-t / 2}|x|^{j} \mathrm{~d} H(x) \\
& +\frac{\left|h^{(m)}(t)\right|}{t^{r} m !} \int_{-t / 2}^{0} \bar{\Delta}_{t,|x| / t}^{r}\left(h^{(m)}\right)|x|^{m+r} \mathrm{~d} H(x) \\
& +H(-t / 2) \sup _{s \geqslant t}|h(s)| .
\end{aligned}
$$

Proof. We first have the inequality

$$
\begin{aligned}
\left|U_{H} h(t)-\int_{-t / 2}^{0} h(t-x) \mathrm{d} H(x)\right| & \leqslant \int_{-\infty}^{-t / 2}|h(t-x)| \mathrm{d} H(x) \\
& \leqslant H(-t / 2) \sup _{s \geqslant t}|h(s)| .
\end{aligned}
$$


Applying Proposition 2.2.5, we have

$$
\begin{aligned}
& \left|\int_{-t / 2}^{0}\left(h(t-x)-\sum_{0 \leqslant j \leqslant m} \frac{(-1)^{j}}{j !} x^{j} h^{(j)}(t)\right) \mathrm{d} H(x)\right| \\
& \leqslant \int_{-t / 2}^{0} \frac{|x|^{m+r}}{t^{r}} \frac{\left|h^{(m)}(t)\right|}{m !} \bar{\Delta}_{t,|x| / t}^{r}\left(h^{(m)}\right) \mathrm{d} H(x) .
\end{aligned}
$$

The result follows.

6.3. A technical lemma. Looking at equality (6.0.1), we need to approximate $U_{H_{I}} \mathcal{A}_{m} \bar{G}_{I}^{(k)}$. The following result, which is the analogue to Lemma 5.5.4 will do. Since $M_{-1} H_{I}$ has the same properties as the distribution function $G$ studied in section 5 , it satisfies the assumptions of the next lemma. Note that even if $M_{-1} F$ is not regularly varying, the assumption $\overline{M_{-1} F}=O(\bar{F})$ and Lemma 5.3.1 - for its part which does not require regular variation - ensure that $H_{I}$ still satisfies the assumption of the following Lemma.

Lemma 6.3.1. Assume that there exist constants $M_{0}$ and $t_{14}$ such that for any integer $k$ less than $\alpha$ and any $t$ at least $t_{14}$,

$$
\int_{-\infty}^{-t}|x|^{k} \mathrm{~d} H(x) \leqslant M_{0} t^{k} F(-t)
$$

and for any nonnegative integer $j$ at most $m$, the moments $\mu_{M_{-1} H, j+r}$ are at most $M_{0}$. Then, there exist a positive $M$ and a $t_{15}$ such that for any $G=\star_{i \geqslant 1} F_{i}$ as in section 5 (that is the distribution function of an infinite weighted sum of nonnegative random variables with nonnegative weights with the assumption of section 5 satisfied), for any $t$ at least $t_{15}$,

$$
\begin{aligned}
& \left|U_{H} \mathcal{A}_{m} \bar{G}^{(k)}-\sum_{i \geqslant 1} L_{H \star G \natural F_{i}, m} \bar{F}_{i}^{(k)}\right|_{k+m, t} \\
& \leqslant M M_{0} C_{\alpha-\epsilon}\left(\bar{\Delta}_{t, v(t) / t}^{r}\left(\bar{F}^{(k+m)}\right)+\bar{\Delta}_{t, 1 / 2}^{r}\left(\bar{F}^{(k+m)}\right) v(t)^{m+r} F(-v(t))\right. \\
& \left.+t^{m} H(-t / 2)\right) .
\end{aligned}
$$

The proof uses an auxiliary result which we state as a claim, very much as we did in the proof of Lemma 5.5.4. 
Claim. For any nonnegative $t$, any distribution function $H$ supported on the nonpositive half line and $K$ supported on the nonnegative half line,

$$
\begin{aligned}
& \left|U_{H} L_{K} h-L_{H \star K} h\right|(t) \\
& \leqslant \sum_{0 \leqslant s \leqslant m} \frac{\left|h^{(s)}(t)\right|}{s !} \sum_{0 \leqslant j \leqslant s}\left(\begin{array}{c}
s \\
j
\end{array}\right) \mu_{K, j} \int_{-\infty}^{-t / 2}|x|^{s-j} \mathrm{~d} H(x) \\
& \quad+\frac{\left|h^{(m)}(t)\right|}{t^{r} m !} \sum_{0 \leqslant j \leqslant m}\left(\begin{array}{c}
m \\
j
\end{array}\right) \mu_{K, j} \int_{-t / 2}^{0} \bar{\Delta}_{t,|x| / t}^{r}\left(h^{(m)}\right)|x|^{m-j+r} \mathrm{~d} H(x) \\
& \quad+H(-t / 2) \sum_{0 \leqslant j \leqslant m} \frac{\mu_{K, j}}{j !} \sup _{s \geqslant t}\left|h^{(j)}(s)\right| .
\end{aligned}
$$

Proof. By linearity of $U_{H}$,

$$
U_{H} L_{K}=\sum_{0 \leqslant j \leqslant m} \frac{(-1)^{j}}{j !} \mu_{K, j} U_{H} \mathrm{D}^{j} .
$$

Applying Lemma 6.2.1 to bound $\left(U_{H}-L_{H, m-j}\right) \mathrm{D}^{j} h$, we obtain

$$
\begin{aligned}
& \left|U_{H} L_{K} h-\sum_{0 \leqslant j \leqslant m} \frac{(-1)^{j}}{j !} \mu_{K, j} L_{H, m-j} h^{(j)}\right|(t) \\
& \leqslant \sum_{0 \leqslant j \leqslant m} \frac{\mu_{K, j}}{j !} \sum_{0 \leqslant l \leqslant m-j} \frac{\left|h^{(l+j)}(t)\right|}{l !} \int_{-\infty}^{-t / 2}|x|^{l} \mathrm{~d} H(x) \\
& \quad+\sum_{0 \leqslant j \leqslant m} \frac{\mu_{K, j}}{j !} \frac{\left|h^{(m)}(t)\right|}{t^{r}(m-j) !} \int_{-t / 2}^{0} \bar{\Delta}_{t,|x| / t}^{r}\left(h^{(m)}\right)|x|^{m-j+r} \mathrm{~d} H(x) \\
& \quad+\sum_{0 \leqslant j \leqslant m} \frac{\mu_{K, j}}{j !} H(-t / 2) \sup _{s \geqslant t}\left|h^{(j)}(s)\right| .
\end{aligned}
$$

Again, Lemma 2.1.4 shows that the left hand side of this inequality is the absolute value of $\left(U_{H} L_{K}-L_{H \star K}\right) h$ evaluated at $t$. Setting $s=l+j$, the right hand side is exactly the upper bound given in the claim.

Proof (of Lemma 6.3.1). The triangle inequality implies the pointwise inequality

$$
\left|U_{H} \mathcal{A}_{m} \bar{G}^{(k)}-\sum_{i \geqslant 1} L_{H \star G \natural F_{i}, m} \bar{F}_{i}^{(k)}\right|
$$




$$
\leqslant \sum_{i \geqslant 1}\left|U_{H} L_{G \natural F_{i}, m} \bar{F}_{i}^{(k)}-L_{H \star G \sharp F_{i}, m} \bar{F}_{i}^{(k)}\right| .
$$

The claim yields for any positive $t$,

$$
\begin{aligned}
& \left|U_{H} L_{G \natural F_{i}} \bar{F}_{i}^{(k)}-L_{H \star G \natural F_{i}} \bar{F}_{i}^{(k)}\right|(t) \\
& \leqslant \sum_{0 \leqslant s \leqslant m} \frac{\left|\bar{F}_{i}^{(k+s)}(t)\right|}{s !} \sum_{0 \leqslant j \leqslant s}\left(\begin{array}{c}
s \\
j
\end{array}\right) \mu_{G \natural F_{i}, j} \int_{-\infty}^{-t / 2}|x|^{s-j} \mathrm{~d} H(x) \\
& \quad+\frac{\left|\bar{F}_{i}^{(k+m)}(t)\right|}{t^{r} m !} \sum_{0 \leqslant j \leqslant m}\left(\begin{array}{c}
m \\
j
\end{array}\right) \mu_{G \natural F_{i}, j} \\
& \quad \times \int_{-t / 2}^{0} \bar{\Delta}_{t,|x| / t}^{r}\left(\bar{F}_{i}^{(k+m)}\right)|x|^{m-j+r} \mathrm{~d} H(x) \\
& +H(-t / 2) \sum_{0 \leqslant j \leqslant m} \frac{\mu_{G \natural F_{i}, j}}{j !} \sup _{s \geqslant t}\left|\bar{F}_{i}^{(k+j)}(s)\right| .
\end{aligned}
$$

We use the same estimates as in the proof of Lemma 5.5.4. So, Lemma 2.2.3 yields

$$
\left|\bar{F}_{i}^{(k+s)}(t)\right| \leqslant M c_{i}^{\alpha-\epsilon} t^{-k-s} \bar{F}(t),
$$

and we also have the moment bound

$$
\mu_{G \natural F_{i}, j} \leqslant \mu_{G, j} \leqslant M .
$$

In (6.3.2), the term

$$
\int_{-t / 2}^{0} \bar{\Delta}_{t,|x| / t}^{r}\left(\bar{F}_{i}^{(k+m)}\right)|x|^{m-j+r} \mathrm{~d} H(x)
$$

is rewritten as a sum of an integral over $[-t / 2,-v(t))$ plus an integral over $[-v(t), 0]$. In this decomposition, the second integral is at most

$$
\bar{\Delta}_{t, v(t) / t}^{r}\left(\bar{F}^{(k+m)}\right) \mu_{M_{-1} H, m-j+r},
$$

while the first one is at most

$$
\bar{\Delta}_{t, 1 / 2}^{r}\left(\bar{F}^{(k+m)}\right) \int_{-\infty}^{-v(t)}|x|^{m-j+r} \mathrm{~d} H(x) .
$$


By assumption, for $t$ at least $2 t_{14}$, the inequality

$$
\int_{-\infty}^{-t / 2}|x|^{s-j} \mathrm{~d} H(x) \leqslant M_{0}(t / 2)^{s-j} F(-t / 2)
$$

holds true. It follows that for $t$ more than some $t_{14}^{\prime}$, the right hand side of (6.3.2) is at most

$$
\begin{aligned}
M c_{i}^{\alpha-\epsilon} t^{-k} \bar{F}(t) F(-t / 2)+ & M c_{i}^{\alpha-\epsilon} t^{-k-m-r} \bar{F}(t)\left(\bar{\Delta}_{t, v(t) / t}^{r}\left(\bar{F}^{(k+m)}\right)\right. \\
& \left.+\bar{\Delta}_{t, 1 / 2}^{r}\left(\bar{F}^{(k+m)}\right) v(t)^{m+r} F(-v(t))\right) \\
+ & M H(-t / 2) c_{i}^{\alpha-\epsilon} t^{-k} \bar{F}(t) .
\end{aligned}
$$

Consequently, for $t$ at least $2 t_{14} \wedge t_{14}^{\prime},(6.3 .1)$ is at most

$$
\begin{aligned}
M C_{\alpha-\epsilon} t^{-k-m} & \bar{F}(t)\left(t^{m} F(-t / 2)+\bar{\Delta}_{t, v(t) / t}^{r}\left(\bar{F}^{(k+m)}\right)\right. \\
& \left.+\bar{\Delta}_{t, 1 / 2}^{r}\left(\bar{F}^{(k+m)}\right) v(t)^{m+r} F(-v(t))+t^{m} H(-t / 2)\right) .
\end{aligned}
$$

For $t$ large enough, $t^{m} F(-t / 2)$ is at most $v(t)^{m+r} F(-v(t))$. This concludes the proof of the lemma.

\subsection{Conditional expansion and removing conditioning. For} any $I$, the distribution functions $M_{-1} H_{I}$ and $G_{I}$ have asymptotic expansions given by the results of section 5 . In particular, $H_{I}$ satisfies the assumptions of Lemma 6.3.1 with a constant $M_{0}$ independent of both $I$ and the sequence $c=\left(c_{i}\right)_{i \geqslant 1}$ which $N_{\alpha, \omega, \gamma}(c)$ at most 1 .

The result of section 5 shows that for some function $\eta(\cdot)$ which tends to 0 at infinity, for any $t$ at least some $t_{13}$, for any set $I$ and any sequence $c$ with $N_{\alpha, \omega, \gamma}(c)$ at most 1 ,

$$
\left|\bar{G}_{I}^{(k)}-\mathcal{A}_{m} \bar{G}_{I}^{(k)}\right|_{k+m, t} \leqslant \eta(t)
$$

where

$$
\mathcal{A}_{m} \bar{G}_{I}^{(k)}=\sum_{i \in \mathbb{N}^{*} \backslash I} L_{G_{I} \sharp F_{+, i}} \bar{F}_{+, i}^{(k)} .
$$

Next, Lemma 6.1.1 shows that ${\overline{H_{I} \star G_{I}}}^{(k)}=U_{H_{I}} \bar{G}_{I}^{(k)}$, which justifies equality (6.0.1). Then, Lemma 6.1.2 and the result of section 5 yield

$$
\left|K^{(k)}-E U_{H_{I}} \mathcal{A}_{m} \bar{G}_{I}^{(k)}\right|_{k+m, t} \leqslant \eta(t) .
$$


Using Lemma 6.3.1, we conclude that there exists a function $\eta^{*}(\cdot)$ with limit 0 at infinity such that

$$
\left|K^{(k)}-E \sum_{i \in \mathbb{N}^{*} \backslash I} L_{H_{I} \star G_{I} \sharp F_{+, i}} \bar{F}_{+, i}^{(k)}\right|_{k+m, t} \leqslant \eta^{*}(t) .
$$

To calculate the expectation involved in the inequality, we rewrite it as

$$
\sum_{i \geqslant 1} E\left(\mathbb{I}\left\{X_{i}>0\right\} L_{H_{I} \star G_{I} \star F_{+, i}, m}\right) \bar{F}_{+, i},
$$

or, equivalently, since the $X_{i}$ 's are independent and identically distributed, as

$$
\sum_{i \geqslant 1} E\left(L_{H_{I} \star G_{I} \dashv F_{+, i}, m} \mid X_{i}>0\right) \bar{F}(0) \bar{F}_{+, i}^{(k)} .
$$

Conditionally upon $X_{i}$ being positive, $H_{I} \star G_{I} \natural F_{i,+}$ is the distribution function of $\sum_{\substack{j \geqslant 1 \\ j \neq i}} c_{i} X_{i}$ given $I$. Thus, the $X_{i}$ 's being indpendent,

$$
E\left(L_{H_{I} \star G_{I} \natural F_{+, i}} \mid X_{i}>0\right)=L_{K \natural F_{i}} .
$$

Next, $\bar{F}(0) F_{+, i}^{(k)}=\bar{F}_{i}^{(k)}$ on the positive half line, and so the expectation that we wanted to calculate is simply $\sum_{i \geqslant 1} L_{K \downarrow F_{i}} \bar{F}_{i}^{(k)}$. This proves Theorem 2.3.1 when the $X_{i}$ 's may assume arbitrary sign and the constants $c_{i}$ 's are nonnegative and $F$ is smooth.

\section{Removing the sign restriction on the constants.}

So far, we proved Theorem 2.3.1 assuming that the $c_{i}$ 's are nonnegative. To drop this requirement is now rather easy, but unfortunately requires some checking which is very much in the flavor of either section 5 or 6 . So we will give the details only for part of the proof. In this section, we keep assuming that $\bar{F}^{(k)}$ and $\bar{M}-1_{-1}(k)$ exist, are bounded and continuous on the positive half line.

We define the set $J$ of all indices pertaining to a positive constant $c_{i}$, that is

$$
J=\left\{i \in \mathbb{N}^{*}: c_{i}>0\right\} .
$$

Define $G$ (resp. $H$ ) to be the distribution function of $\sum_{i \in J} c_{i} X_{i}$ (resp. $\sum_{i \in \mathbb{N}^{*} \backslash J} c_{i} X_{i}$ ). The tail expansion of $\bar{G}^{(k)}$ is given by the result of section 6 . That of $\bar{H}^{(k)}$ follows by the same token, since

$$
\sum_{i \in \mathbb{N}^{*} \backslash J} c_{i} X_{i}=\sum_{i \in \mathbb{N}^{*} \backslash J}\left(-c_{i}\right)\left(-X_{i}\right) .
$$


Now, the distribution function $K$ of $\langle c, X\rangle$ is the convolution $G \star H$.

To obtain an asymptotic expansion of $\overline{G \star H}(k)$, we have two strategies. One is to decompose the distribution functions by conditioning with respect to signs, as we did in section 6; another one is to go more along the line of section 5. Both routes are about equal length, and we go for the latter.

Our starting point is Proposition 5.1.1 which asserts that

$$
\bar{K}=T_{G, 1 / 2} \bar{H}+T_{H, 1 / 2} \bar{G}+M_{2} \bar{G} M_{2} \bar{H}
$$

and for a positive integer $k$,

$$
\bar{K}^{(k)}=T_{G, 1 / 2} \bar{H}^{(k)}+T_{H, 1 / 2} \bar{G}^{(k)}+\sum_{1 \leqslant i \leqslant k-1} M_{2} \bar{G}^{(i)} M_{2} \bar{H}^{(k-i)} .
$$

7.1. Neglecting terms involving the multiplication operators. Starting with (7.0.1), the purpose of this subsection is to show that an expansion of $K^{(k)}$ can be obtained from the ones for $T_{G, 1 / 2} \bar{H}^{(k)}$ and $T_{H, 1 / 2} \bar{G}^{(k)}$, or, equivalently, that the terms $M_{2} \bar{G}^{(i)} M_{2} \bar{H}^{(k-i)}$ can be neglected.

Our first lemma is an analogue of Lemma 6.1.2. The operators $M_{c}$ are not contraction, but for our problems, they behave very much as if they were bounded, which is good enough.

Lemma 7.1.1. There exists $t_{16}$ such that for any positive real number $c$ and any positive $t$ with $(t / c) \wedge t$ at least $t_{16}$,

$$
\left|M_{c} h\right|_{p, t} \leqslant\left(c^{p+\alpha+1} \vee c^{p+\alpha-1}\right)|h|_{p, t / 2} .
$$

Proof. We simply write

$$
\frac{s^{p}}{\bar{F}(s)} h(s / c)=\frac{(s / c)^{p} h(s / c)}{\bar{F}(s / c)} c^{p} \frac{\bar{F}(s / c)}{\bar{F}(s)},
$$

from which we deduce

$$
\left|M_{c} h\right|_{p, t} \leqslant c^{p}|h|_{p, t / c} \sup _{s \geqslant t} \bar{F}(s / c) / \bar{F}(s) .
$$

We conclude in using Lemma 2.2.4. 
Our next lemma states a rather obvious property of the norm $|\cdot|_{p, t}$.

Lemma 7.1.2. For any positive real $p, q$ and $t$,

$$
|f g|_{p+q+m, t} \leqslant|f|_{p, t}|g|_{q, t} \sup _{s \geqslant t} s^{m} \bar{F}(s) .
$$

Proof. It follows from the identity

$$
\frac{s^{p+q+m}}{\bar{F}(s)} f(s) g(s)=\frac{s^{p}}{\bar{F}(s)} f(s) \frac{s^{q}}{\bar{F}(s)} g(s) s^{m} \bar{F}(s) .
$$

Our first two lemmas in this subsection imply the following bound.

Lemma 7.1.3. Assume that the hypotheses of Theorem 2.3.1 hold. Suppose also that $F^{(k)}$ exists, is continuous and bounded. Then, there exists a function $\eta^{*}(\cdot)$ with limit 0 at infinity and a positive number $t_{17}$ such that for any $t$ at least $t_{17}$ and any sequence $c$ with $N_{\alpha, \omega, \gamma}(c)$ at most 1 ,

$$
\left|\sum_{1 \leqslant i \leqslant k-1} M_{2} \bar{G}^{(i)} M_{2} \bar{H}^{(k-i)}\right|_{k+m, t} \leqslant \eta^{*}(t)
$$

Proof. The triangle inequality, Lemmas 7.1.1 and 7.1.2 yield for $t$ at least 1 ,

$$
\begin{aligned}
& \left|\sum_{1 \leqslant i \leqslant k-1} M_{2} \bar{G}^{(i)} M_{2} \bar{H}^{(k-i)}\right|_{k+m, t} \\
& \quad \leqslant \sum_{1 \leqslant i \leqslant k-1} 2^{k+m+\alpha+1}\left|\bar{G}^{(i)}\right|_{i, t / 2}\left|\bar{H}^{(k-i)}\right|_{k-i, t / 2} \sup _{s \geqslant t / 2} s^{m} \bar{F}(s) .
\end{aligned}
$$

From the result of section 6 ,

$$
\left|\bar{G}^{(i)}-\mathcal{A}_{0} \bar{G}^{(i)}\right|_{i, t / 2} \leqslant \eta(t / 2) .
$$

Since

$$
\mathcal{A}_{0} \bar{G}^{(i)}=\sum_{n \in J} \bar{F}_{n}^{(i)}
$$


the triangle inequality and lemma 2.2.3 imply that for $t$ at least $t_{1}$,

$$
\left|\mathcal{A}_{0} \bar{G}^{(i)}(t)\right| \leqslant M \sum_{n \in J} c_{n}^{\alpha-\epsilon} t^{-i} \bar{F}(t) .
$$

Consequently,

$$
\left|\bar{G}^{(i)}\right|_{i, t / 2} \leqslant \eta(t / 2)+M C_{\alpha-\epsilon} .
$$

We have a similar bound for $\left|H^{(k-i)}\right|_{k+m, t / 2}$. Since $s^{m} \bar{F}(s)=o(1)$ as $s$ tends to infinity, the result follows.

7.2. Substituting $\bar{H}^{(k)}$ and $\bar{G}^{(k)}$ by their expansions. Looking at equality (7.0.1), we would like to use Theorem 5.4.1 to conclude that $T_{G, 1 / 2} \bar{H}^{(k)}$ has asymptotic expansion $L_{G_{m}} \bar{H}^{(k)}$. This is not possible since we do not know if $\bar{H}^{(k)}$ is smoothly varying - the problem is not so much about the regular variation part of the smoothly varying condition, but more about the continuity of $\bar{H}^{(k+m)}$. The trick is to replace first $\bar{H}^{(k)}$ by its asymptotic expansion. Since the expansion involves explicitely $\bar{F}$ and its derivatives, we will be able to study the action of $T_{G, 1 / 2}$ on the expansion. The first step is to show that $T_{G, 1 / 2}$ behaves like a bounded operator in our problem (though it is not.)

Lemma 7.2.1. For $t$ at least $t_{1}$,

$$
\left|T_{G, 1 / 2} h\right|_{p, t} \leqslant 2^{p+\alpha+1}|h|_{p, t / 2} .
$$

Proof. It follows the proof of Lemma 5.5.2.

This lemma and the result of section 6 imply

$$
\left|T_{G, 1 / 2} \bar{H}^{(k)}-T_{G, 1 / 2} \mathcal{A}_{m} \bar{H}^{(k)}\right|_{k+m, t} \leqslant 2^{k+m+\alpha+1} \eta(t / 2) .
$$

Of course a similar inequality holds if we permute $G$ and $H$.

To conclude the proof of Theorem 2.3.1 when $F$ is smooth, we write

$$
T_{G, 1 / 2} \mathcal{A}_{m} \bar{H}^{(k)}=\sum_{n \in \mathbb{N} * \backslash J} T_{G, 1 / 2} L_{H \natural F_{n}, m} \bar{F}_{n}^{(k)} .
$$


Since $L_{H \natural F_{n}, m} \bar{F}_{n}^{(k)}$ involves derivatives of $\bar{F}_{n}$, it then suffices to study the expansion of $T_{G, 1 / 2} \bar{F}_{n}^{(k)}$ and have a good error bound in this expansion. This is now straightfoward. Indeed, write

$$
T_{G, 1 / 2} \bar{F}_{n}^{(k)}=\int_{-\infty}^{-t / 2} \bar{F}_{n}(t-x) \mathrm{d} G(x)+\int_{-t / 2}^{t / 2} \bar{F}_{n}^{(k)}(t-x) \mathrm{d} G(x) .
$$

We then bound

$$
\begin{aligned}
\left|\int_{-\infty}^{-t / 2} \bar{F}_{n}^{(k)}(t-x) \mathrm{d} G(x)\right| & \leqslant G(-t / 2) \sup _{s \geqslant t / 2}\left|\bar{F}_{n}^{(k)}(s)\right| \\
& \leqslant M\left|c_{n}\right|^{k} G(-t / 2) t^{-k} F(-t / 2),
\end{aligned}
$$

and we expand

$$
\int_{-t / 2}^{t / 2} \bar{F}_{n}^{(k)}(t-x) \mathrm{d} G(x)
$$

into $L_{G, m} \bar{F}^{(k)}$ as we did in sections 5 and 6 .

This completes the proof of Theorem 2.3.1 when $F^{(k)}$ is continuous and bounded.

\section{Removing the smoothness assumption.}

We now want to remove the assumption that $\bar{F}^{(k)}$ exist, is continuous and bounded on the whole real line.

In Theorem 2.3.1, the membership of $\bar{F}$ and $\overline{M_{-1} F}$ to $S R_{-\alpha, \omega}$ ensures that there exists some $A$ more than 2 say such that $\bar{F}^{(k)}$, $\bar{M}-1 F^{(k)}$ exist, are continuous and bounded on $(A, \infty)$. Thus, we can write $F=(1-p) F_{0}+p F_{1}$ where $F_{0}$ is concentrated on $(-A-1, A+1)$ and $F_{1}$ is concentrated on $(-A, A)^{c}$, and such that $F_{1}^{(k)}$ and $\overline{M_{-1} F_{1}}{ }^{(k)}$ exist, are continuous and bounded on the whole real line. Let $\epsilon_{i}$ be a sequence of independent Bernoulli random variable with mean $p$. Let $I$ be the random set of integer $i$ for which $\epsilon_{1}$ is 1 . Given $I$, let $G_{I}$ be $\star_{i \in \mathbb{Z} \backslash I} M_{c_{i}} F$ and $H_{I}$ be $\star_{i \in I} M_{c_{i}} F_{0}$. Then $\star_{i \in \mathbb{Z}} M_{c_{i}} F_{1}$ is $E G_{I} \star H_{I}$. Since the $c_{i}$ 's are summable, the support of the distributions pertaining to $G_{I}$ is included in $|c|_{1}[-A-1, A+1]$ no matter what $I$ is. it follows that on $2|c|_{1}(A+1, \infty)$,

$$
\overline{G_{I} \star H_{I}}=T_{G_{I}} \overline{H_{I}} .
$$

The expansion for $\overline{G_{I} \star H_{I}}$ follows from that of $H_{I}$ using the same arguments that we used in sections 6 and 7 . This concludes the proof of Theorem 2.3.1. 
Appendix. Maple code. The goal in writing the following Maple code was to see to which extend the tail calculus described in section 3.2 could be automatized. With this code, the user enters a distribution function having an expansion on some power of $t^{-1}$ and the integer $m$ occuring in Theorem 2.3.1. The program then generates the coefficients of the asymptotic expansion of $G_{c}$ (with the notation of Theorem 2.3.1), assuming that the $c_{i}$ 's are positive.

The example which we ran here is that of the Burr distribution in subsection 3.2.

The tail $\bar{F}$ is of (for 'overlined $F^{\prime}$ ). One needs to specify the parameter $\alpha$, which is named palpha and $m$.

restart; with(LinearAlgebra):

pgamma: $=10:$ ptau $:=3 / 2$ :

oF $:=\left(1+\left(t^{\wedge}(\right.\right.$ ptau $\left.)\right) /$ beta $) \wedge(-$ pgamma $)$;

palpha:=ptau*pgamma; $\mathrm{m}:=4$;

We then expand the tail of $F$. We write the tail expansion as a polynomial of $x=1 / t$.

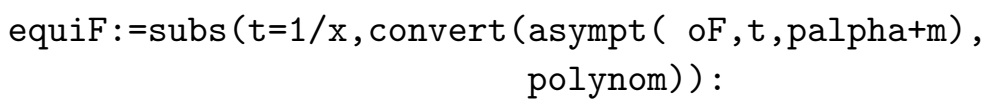

We build the set of powers of $x$ involved in this expansion.

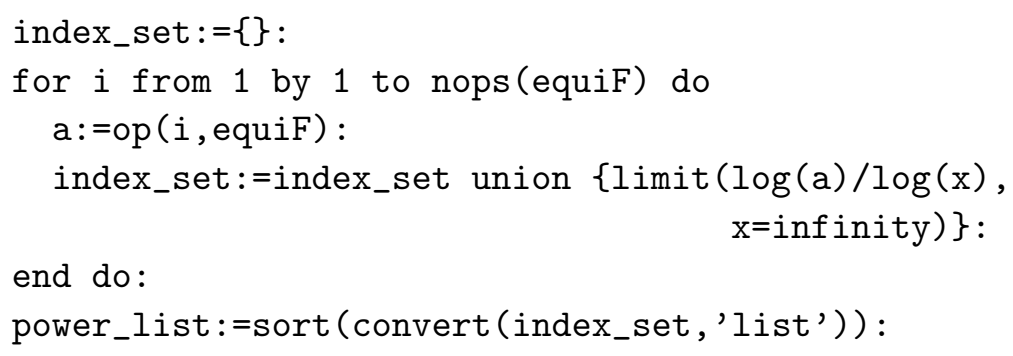

We complete this list by the augmentation procedure described in section 3.2.

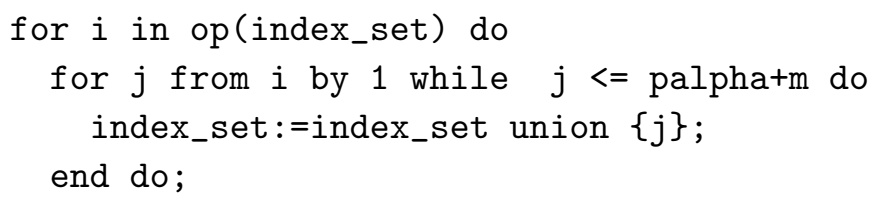


end do;

index_list: $=$ sort $($ convert (index_set, 'list')) :

Then we calculate the dimension of the vector space we will work with.

vdim:=nops (index_list) :

We calculate the vector $p_{\bar{F}}$. Unfortunately, with Maple, it is easy to work with polynomials, but it has no useful command to work with monomials of noninteger degree. That induces the following code where we obtained the monomials one by one.

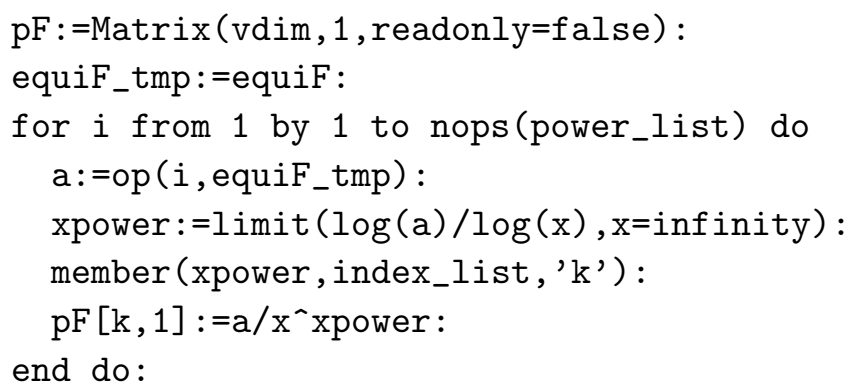

The next step is to build the matrices $\mathcal{D}$ and $\mathcal{M}_{c}$, which we call Dmat and Mcmat in the code.

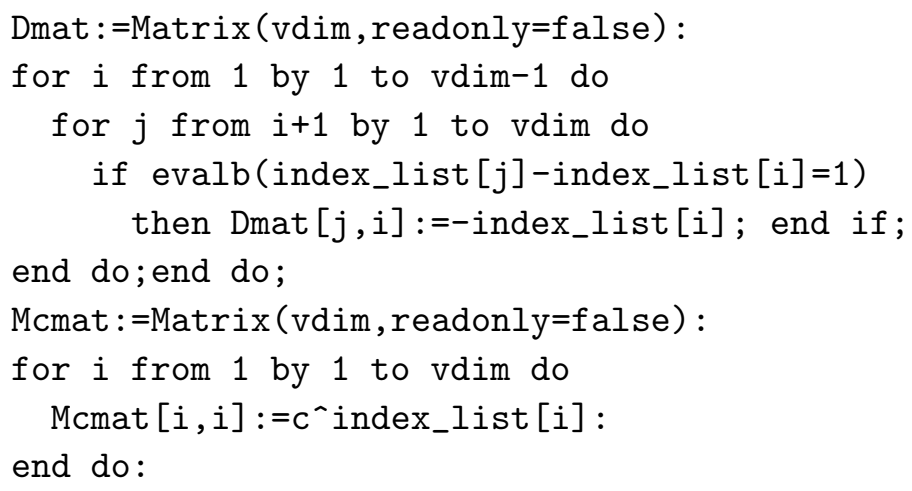

We construct the Laplace character of $F$ in its matrix form $\mathcal{L}_{F}$. The matrix is Lmat.

Lmat : =Matrix (vdim, readonly=false $)$ : 


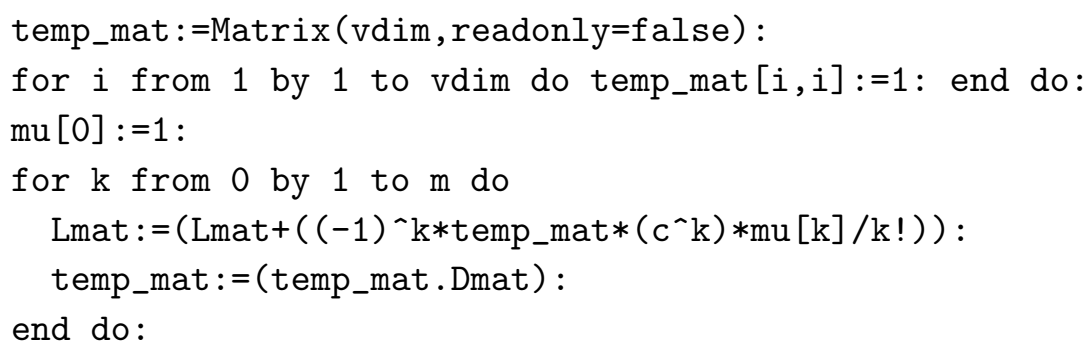

Its inverse is

Lmat_inv:=MatrixInverse (Lmat, method='subs') :

We write the expansion for $G$ as $\mathcal{L}_{G} \sum_{i \in \mathbb{Z}} \mathcal{L}_{F}^{-1} p_{\overline{M_{c_{i}} F}}$. We calculate the generic summands $\mathcal{L}_{F}^{-1} p_{\overline{M_{c} F}}$.

Msum : =Lmat_inv $\cdot$ Mcmat $\cdot \mathrm{pF}$ :

To sum these summands amounts to substitute $c^{p}$ by $C_{p}$ in this sum, we we do now.

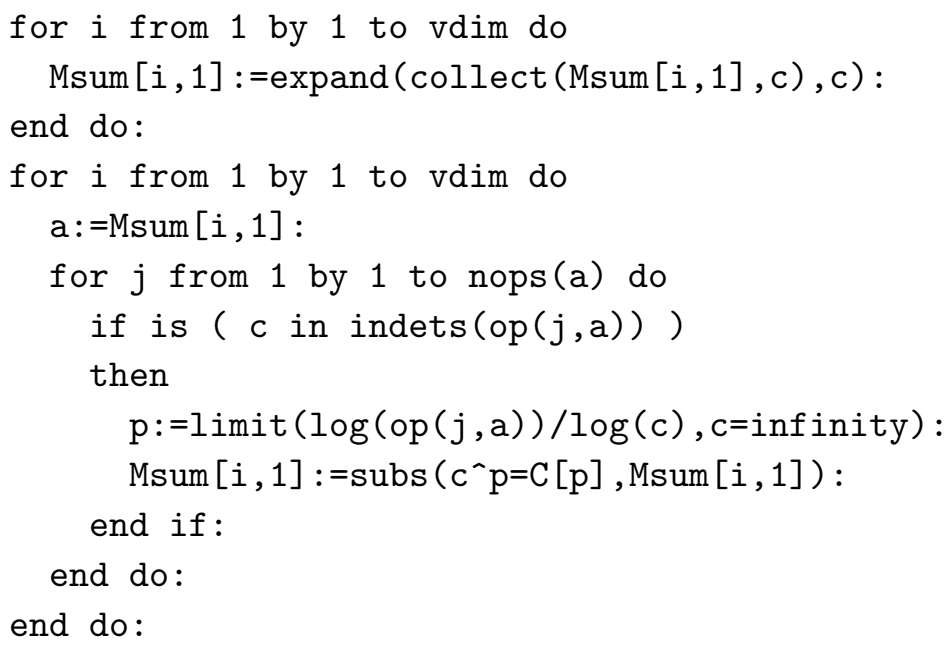

To calculate the Laplace character of $G_{c}$, we first obtain its moments. The algorithm is that described in Barbe and McCormick (2005). These moments are coded as muG $[\mathrm{k}]$.

for $k$ from 0 by 1 to $m$ do 


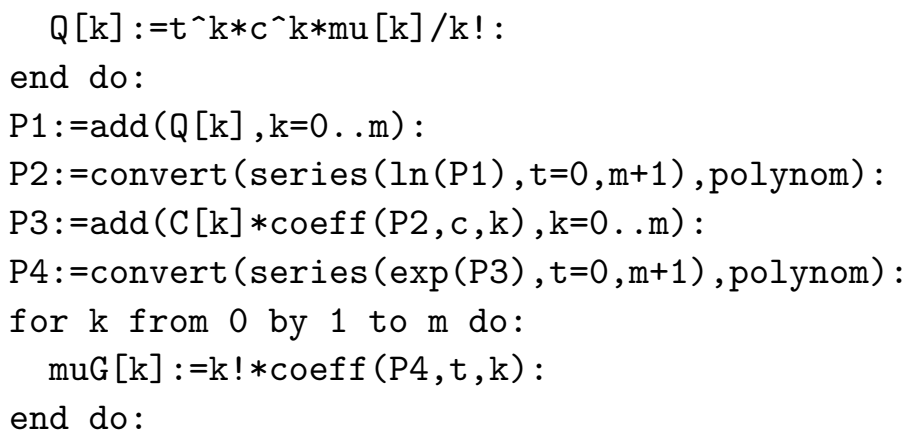

We construct the Laplace character of $G$.

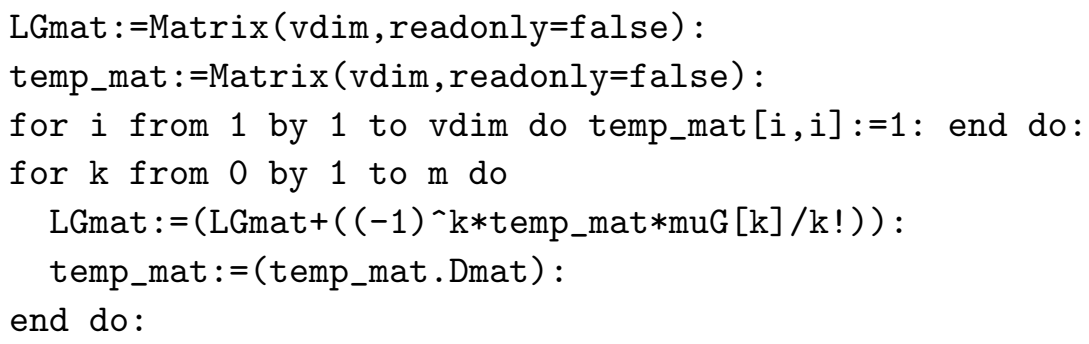

And finally obtain the coefficient of the tail, that is $p_{\bar{G}}$.

tail :=LGmat . Msum:

In our example for the Burr distribution, these coefficients are messy. The remaining code makes them nicer looking; at least looking good enough so that they can be written as we did.

The first step in the simplification is to substitute the centered moments for the noncentered ones. In the code, we write kappa [k] the $k$-the centered moment. We express it as a function of the noncentered ones.

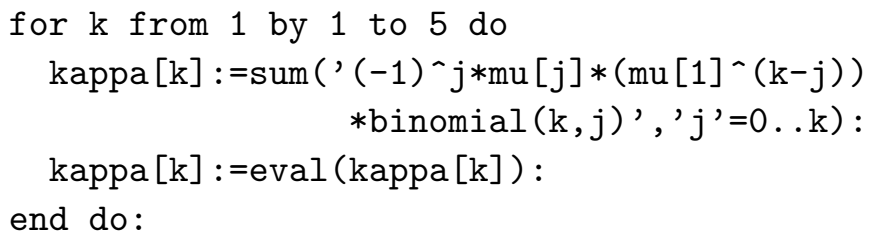

Then we solve inductively for the noncentered moments, thereby expressing them as functions of the centered ones. 


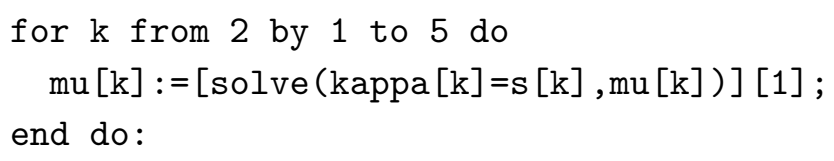

Finally we do the substitution and arrange the terms by powers of $\beta$.

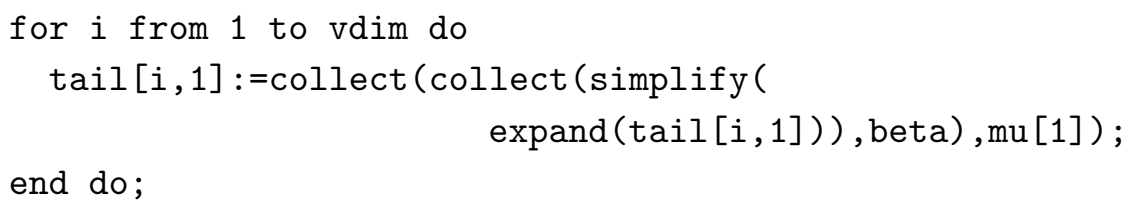

Acknowledgements. Philippe Barbe thanks Peter Haskell and Konstantin Mischaikow for changing his views on algebraic constructions through their marvelous teaching.

\section{References}

J. Abate, G.L. Choudhury, D.M. Lucantoni, W. Whitt (1995). Asymptotic analysis of tail probabilities based on computation of moments, Ann. Appl. Probab., 5, 983-1007.

J. Abate, G.L. Choudhury, W. Whitt (1994). Waiting-time tail probabilities in queues with long-tail service-time distribution, Queueing Systems Theory Appl., 16, 311-338.

J. Abate, W. Whitt (1997). Asymptotics for M/G/1 low-priority waiting-time tail probability, Queueing Systems Theory Appl., 25, 173-233.

K.B. Athreya, P. Ney (1972). Branching Processes, Springer.

Ph. Barbe, W.P. McCormick (2005). Asymptotic expansions of convolutions of regularly varying distributions, J. Austr. Math. Soc., to appear.

Ph. Barbe, W.P. McCormick (200?). Tail calculus with remainder, applications to tail expansions for infinite order moving averages, randomly stopped sums, and related topics, submitted.

J. Beirlant, J.L. Teugels, P. Vynckier (1996). Practical Analysis of Extreme Values, Leuven University Press, Leuven, Belgium.

N.H. Bingham, C.M. Goldie, J.L. Teugels (1989) Regular Variation, 2nd ed., Cambridge 
R. Bojanic, J. Karamata (1963). On slowly varying functions and asymptotic relations, Math. Research Center Tech. Report, 432, Madison, Wisconsin.

A.A. Borovkov, K.A. Borovkov (2003). On large deviation probabilities for random walks, I, regularly varying distribution tails, Theory Probab. Appl., 46, 193-213.

L. Breiman (1968). Probability, Addison-Wesley.

P.J. Brockwell, R.A. Davis (1991). Time Series: Theory and Methods, 2nd ed., Springer.

M. Broniatowski, A. Fuchs (1995). Tauberian theorems, Chernoff inequality, and the tail behavior of finite convolutions of distribution functions, Adv. Math., 116, 12-33.

J. Chover, P. Ney, S. Wainger (1973). Functions of probability measures, J. Analyse Math., 26, 255-302.

Y.S. Chow, H. Teicher (1978). Probability Theory, Independence, Interchangeability, Martingales, Springer.

J.W. Cohen (1972). On the tail of the stationary waiting-time distribution and limit theorem for $\mathrm{M} / \mathrm{G} / 1$ queue, Ann. Inst. $H$. Poincaré, B, 8, 255-263.

R.A. Davis, M. Rosenblatt (1991). Parameter estimation for some time series models without contiguity, Statist. Prob. Lett., 11, $515-521$.

J. Delsarte (1938). Sur une extension de la formule de Taylor, Journ. Math. Pures et Appl., 28(3), 213-231.

P. Diaconis, D. Freedman (1999). Iterated random functions, SIAM Review, 41, 45-70.

R.M. Dudley (1989). Real Analysis and Probability, Chapman \& Hall.

P. Embrechts, C.M. Goldie, N. Veraverbeke (1979). Subexponentiality and infinite divisibility, Z. Wahrsch. verw. Geb., 49, 335-347.

P. Embrechts, C. Klüppelberg, T. Mikosch (1997). Modelling Extremal Events, Springer.

W. Feller (1971). An Introduction to Probability Theory and Its Applications, vol. 2, Wiley.

J.L. Geluk (1992). Second order tail behaviour of a subordinated probability distribution, Stoch. Proc. Appl., 40, 325-337.

J.L. Geluk (1994). Asymptotic behaviour of the convolution tail of distributions each having a first or second order regularly varying tail, Analysis, 14, 163-183. 
J.L. Geluk (1996). Tails of subordinated laws: the regular varying case, Stoch. Proc. Appl., 61, 147-161.

J.L. Geluk, L. de Haan, S. Resnick, C. Starica (1997). Secondorder regular variation, convolution and the central limit theorem, Stoch. Proc. Appl., 69, 139-159.

J. Geluk, L. Peng, C.G. De Vries (2000). Convolutions of heavytailed random variables and applications to portfolio diversification and MA(1) time series, Adv. Appl. Prob., 32, 1011-1026.

C.M. Goldie (1991). Implicit renewal theory and tails of solutions of random equations, Ann. Appl. Probab., 1, 126-166.

D.R. Grey (1994). Regular variation in the tail behavior of solutions of random difference equations, Ann. Probab., 4, 169-183.

A.K. Grincevičius (1975). On limit distribution for a random walk on the line, Lithuanian Mat. J., 15, 580-589.

R. Grübel (1987). On subordinated distributions and generalized renewal measures, Ann. Probab., 15, 394-415.

P. Hall, I. Weissman (1997). On the estimation of extreme tail probabilities, Ann. Statist., 25, 1311-1326.

B.M. Hill (1973). A simple general approach to inference about the tail of a distribution, Ann. Statist., 3, 1163-1174.

H. Kesten (1973). Random difference equations and renewal theory for product of random matrices, Acta. Math., 131, 207-248.

L. Mattner (2004). Cumulants are universal homomorphisms into Hausdorff groups, Probab. Theor. Rel. Fields, 130, 151-166.

V. Marić (2000). Regular Variation and Differential Equations, Lecture Notes in Mathematics, 1726, Springer.

V. Marić, M. Tomić (1977). Regular variation and asymptotic properties of solutions of nonlinear differential equations, Publ. Inst. Math. (Beograd), 21 (35), 119-129.

A. Nijenhuis, H. Wilf (1978). Combinatorial Algorithms, Academic Press, second edition.

F.W.J. Olver (1974). Asymptotic and Special Functions, Academic Press.

E. Omey (1981). Regular variation and its applications to second order linear differential equations, Bull. Soc. Math. Belg., 32, 207229 .

E. Omey (1988). Asymptotic properties of convolution products of functions, Publ. Inst. Math. (Beograd) (N.S.), 43, 41-57.

E. Omey, E. Willekens (1986). Second order behavior of the tail of a subordinated probability distribution, Stoch. Proc. Appl., 21, 
339-353.

E. Omey, E. Willekens (1987). Second order behavior of distributions subordinated to a distribution with finite mean, Comm. Statist. Stoch. Models, 3, 311-342.

A.G. Pakes (1975). On the tails of waiting-time distributions, J. Appl. Probab., 12, 555-564.

A.G. Pakes (2004). Convolution equivalence and infinite divisibility, J. Appl. Probab., 41, 407-424.

S. Resnick (1986). Point processes, regular variation and weak convergence, Adv. Appl. Probab., 18, 66-138.

S. Resnick (1987). Extreme Values, Regular Variation, and Point Processes, Springer.

S. Resnick, C. Stărică (1997). Asymptotic behavior of Hill's estimator for autoregressive data, heavy tails and highly volatile phenomena, Comm. Statist., Stoch. Models, 13, 703-721.

S. Resnick, E. Willekens (1991). Moving averages with random coefficients and random coefficients autoregressive models, Comm. Statist. Stoch. Models, 7, 511-525.

T. Rolski, H. Schmidli, V. Schmidt, J. Teugels (1999). Stochastic Processes for Insurance and Finance, Wiley.

B. Solomyak (1995). On the random series $\sum \pm \lambda^{n}$ (an Erdös problem), Ann. Math., 142, 611-625.

D. Stanton, D. White (1986). Constructive Combinatorics, Springer.

E. Willekens (1989). Asymptotic approximation of compound distributions and some applications, Bull. Soc. Math. Belg., ser. B, $41,55-61$.

E. Willekens, J.L. Teugels (1992). Asymptotic expansions for waiting time probabilities in an $\mathrm{M} / \mathrm{G} / 1$ queue with long-tailed service time, Queueing Systems Theory Appl., 10, 295-311.

G.E. Willmot, X.S. Lin (2000). Lundberg Approximations for Compound Distributions with Insurance Applications, Lecture Notes in Statistics, 156, Springer.

Ph. Barbe

90 rue de Vaugirard

75006 PARIS

FRANCE
W.P. McCormick

Dept. of Statistics

University of Georgia

Athens, GA 30602

USA

bill@stat.uga.edu 